\title{
Variable Length Windowing to Improve Non-Negative Matrix Factorization of Music Signals
}

\author{
by \\ Revanth Pentyala, B.Eng. \\ A thesis submitted to the \\ Faculty of Graduate and Postdoctoral Affairs \\ in partial fulfillment of the requirements for the degree of
}

Master of Applied Science in Electrical Engineering

Ottawa-Carleton Institute for Electrical and Computer Engineering

Department of Systems and Computer Engineering

Carleton University

Ottawa, Ontario

August, 2015

(C) Copyright

Revanth Pentyala, 2015 
The undersigned hereby recommends to the

Faculty of Graduate and Postdoctoral Affairs acceptance of the thesis

\title{
Variable Length Windowing to Improve Non-Negative Matrix Factorization of Music Signals
}

\author{
submitted by Revanth Pentyala, B.Eng. \\ in partial fulfillment of the requirements for the degree of \\ Master of Applied Science in Electrical Engineering
}

Professor Yvan Labiche, Chair,

Department of Systems and Computer Engineering

Ottawa-Carleton Institute for Electrical and Computer Engineering

Department of Systems and Computer Engineering

Carleton University

August, 2015 


\section{Abstract}

Non-negative matrix factorization(NMF) has shown positive results in learning musical notes over other blind source separation techniques like independent component analysis (ICA) and principal component analysis (PCA). The magnitude short time Fourier transform (STFT) is typically given as input for the non-negative matrix factorization algorithm for learning. Non-negative matrix factorization treats each frame of the magnitude short time Fourier transform as independent and identically distributed vectors. Due to fixed length windowing, a single note might be spread across multiple frames with varying proportions of the note structure. Improving the stationarity characteristics of the signal within the STFT is expected to improve the qualitative performance of note extraction by the NMF algorithm. To this extent, we propose a signal dependent variable length window based STFT to effectively capture the stationarity of the signal within each frame of the magnitude STFT.

In this thesis, automatic detection of note onsets in music is studied. Many reduction techniques have been developed in the literature for reducing the timefrequency representation of the signal to a one dimension detection function to detect note onsets. We have come up with an onset detection technique that makes few assumptions about underlying structure of music and works well across a wide variety of music. A novel approach to extract note onset timings using the Itakura-Saito divergence is proposed.

The remainder of the thesis explores the use of a variable length window based STFT versus the fixed length window based STFT for NMF decomposition, and how the Itakura-Saito divergence based onset detection works in comparison to other techniques. 


\section{Acknowledgments}

I would like to thank my supervisor Dr. Richard M. Dansereau (Systems and Computer Engineering, Carleton University) for his patience, insight, support and guidance through the course of this thesis and my colleague Ce Peng for his valuable advice. I would like to extend my thanks to Sean Kormilo and Tony Wacheski (CTO and CEO, Anystone Technologies Inc.) for providing valuable experience and feedback. I would like to thank Carleton University for providing me with this great opportunity.

Lastly, I would like to thank my family and friends for their invaluable support. 


\section{Table of Contents}

Abstract $\quad$ iii

Acknowledgments $\quad$ iv

Table of Contents $\quad$ v

List of Tables viii

List of Figures $\quad$ ix

1 Introduction $\quad 1$

1.1 Motivation . . . . . . . . . . . . . . . . 1

1.2 General Approaches to Note Extraction . . . . . . . . . . . . . . . 2

1.3 Proposed Approach to Note Extraction . . . . . . . . . . . . . . . 3

1.4 General Approaches to Detect Note Onsets . . . . . . . . . . . . . . 4

1.5 Proposed Approach to Detect Onset Timings . . . . . . . . . . . . . 4

1.6 Contributions . . . . . . . . . . . . . . . . . 5

1.7 Thesis Organization . . . . . . . . . . . . . . . . . 5

2 Background and Foundational Information 6

2.1 Structure of Music . . . . . . . . . . . . . . . . 6

2.2 Short Time Fourier Transform . . . . . . . . . . . . . . . . 6

2.2.1 Discrete Fourier Transform . . . . . . . . . . . . . . . . 7

2.2.2 Forward Short Time Fourier Transform . . . . . . . . . . . . . 8

2.2.3 Choice of Analysis Window and Length . . . . . . . . . . . . . 10

2.2.4 Choice of FFT Length . . . . . . . . . . . . . . . . . . . 11

2.2.5 Choice of Stride . . . . . . . . . . . . . . . . . . . . . . 11

2.2.6 Time Frequency Resolution of STFT . . . . . . . . . . . . . . 12 
2.3 Introduction to Distance Measures . . . . . . . . . . . . . . 13

2.4 Onset Detection Function _. . . . . . . . . . . . . . . . . 14

2.4.1 Scheme of Onset Detection . . . . . . . . . . . . . . . 14

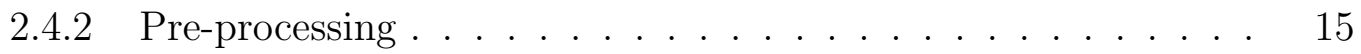

2.4 .3 Reduction . . . . . . . . . . . . . . . . . 17

2.4 Peak Selection . . . . . . . . . . . . . . . 20

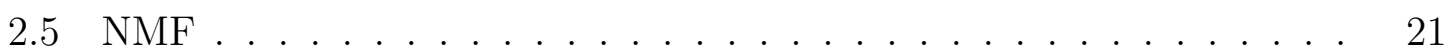

$2.5 .1 \quad$ NMF on Music . . . . . . . . . . . . . . . . . . . 23

3 Onset Detection Function $\quad 25$

3.1 Methodology/Algorithm . . . . . . . . . . . . . . . . 25

3.1 .1 Pre-processing . . . . . . . . . . . . . . . . . . . . 25

3.1 .2 Reduction . . . . . . . . . . . . . . . . . 27

3.1 .3 Post-Processing . . . . . . . . . . . . . . . . . . . . 28

3.2 Experimental Setup and Results . . . . . . . . . . . . . . . 28

3.3 Discussions . . . . . . . . . . . . . . . . . . . . . . . . . 40

4 Variable Window Based STFT for NMF Decomposition 45

4.1 Methodology/Algorithm . . . . . . . . . . . . . . . . 47

4.1.1 Variable Length Window Based STFT . . . . . . . . . . 47

4.1.2 Fixed vs Variable Length Window Based STFT . . . . . . . 47

4.2 Implementation of $\mathrm{NMF} \ldots \ldots \ldots \ldots \ldots$

4.3 Experimental Setup and Discussion on Results . . . . . . . . . . 52

$4.3 .1 \quad$ Experimental Setup . . . . . . . . . . . . . . . . . . 52

4.3 .2 Analysis of $\mathbf{W}$ Matrix . . . . . . . . . . . . . . . . 53

4.3 .3 Analysis of $\mathbf{H}$ Matrix . . . . . . . . . . . . . . . . . 57

4.3.4 Similarity of Derived Notes with Ground Truth Notes . . . . . 58

4.3.5 Analysis of Factorization of Complex Signal . . . . . . . . 61

4.3.6 Perceptual Evaluation of Derived Notes . . . . . . . . . . 67

4.3.7 Understanding the Results . . . . . . . . . . . . . . . 70

5 Conclusion and Future Work $\quad 72$

5.1 Itakura-Saito Divergence Based Onset Detection . . . . . . . . . 72

5.2 Variable Length Window Based STFT _. . . . . . . . . . 72

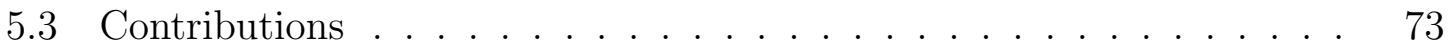


5.4 Future Work . . . . . . . . . . . . . . . . . . . . . 73

$\begin{array}{ll}\text { References } & 75\end{array}$ 


\section{List of Tables}

1 Distribution and distance measures . . . . . . . . . . . . . 14

2 Performance of various onset detection methods compared to the proposed method for music file containing simple piano . . . . . . . . . 31

3 Performance of various onset detection methods compared to the proposed method for music file containing complex piano . . . . . . . . 32

4 Performance of various onset detection methods compared to the proposed method for music file containing simple violin . . . . . . . . . 33

5 Performance of various onset detection methods compared to the proposed method for music file containing complex violin . . . . . . . 34

6 Performance of various onset detection methods compared to the proposed method for music file containing simple flute . . . . . . . . 35

7 Performance of various onset detection methods compared to the proposed method for music file containing complex flute . . . . . . . 36

8 Performance of various onset detection methods compared to the proposed method for music file containing simple guitar . . . . . . . . 37

9 Performance of various onset detection methods compared to the proposed method for music file containing complex guitar . . . . . . . 38

10 Performance of various onset detection methods compared to the proposed method for music file containing mixed signal . . . . . . . . 39

11 Bhattacharyya distance as similarity measure between derived notes in

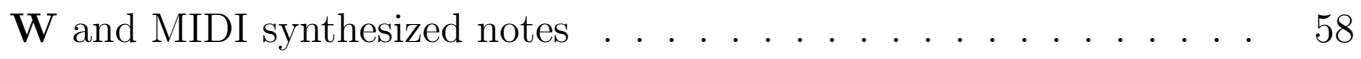

12 ITU-R five-grade impairment scale . . . . . . . . . . . . . . . . 69

13 PEAQb scores for the reconstructed audio signal from $\mathbf{W}$ and $\mathbf{H}$ matrices 69 


\section{List of Figures}

1 Parts of a note in time domain signal $\ldots \ldots \ldots \ldots \ldots$

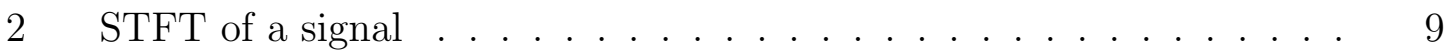

3 (a) Wideband vs (b) narrowband short time Fourier transform . . . . 12

4 Onset detection flowchart . . . . . . . . . . . . . . 15

5 Scheme of onset detection depicting (a) time domain signal (b) short time Fourier transform of the time domain signal (c) detection function for note onsets, and (d) note onsets detected . . . . . . . . . . 16

6 Example of NMF decomposition . . . . . . . . . . . . . . . . . 24

7 Onset detection flowchart $\ldots \ldots \ldots \ldots \ldots$

8 Performance of various distance measures . . . . . . . . . . . . . 40

9 Performance of $D_{I S}\left(X_{n} \| \hat{X}_{n}\right)$ for on a segment of a music clip with no vocals (Real Love by Clean Bandit): (a) time series plot, (b) spectrogram, and (c) plot of $D_{I S}\left(X_{n} \| \hat{X}_{n}\right) \ldots \ldots \ldots \ldots \ldots$

10 Variable length window based NMF scheme for music note separation 46

11 (a) Example time-domain plot of simple music signal. (b) Onset detection of notes for signal dependent variable length windowing. (c) Time domain signal between two consecutive onsets is segmented for signal dependent variable length window based STFT. . . . . . . . 49

12 Fixed length window based STFT . . . . . . . . . . . . . 50

13 Variable length window based STFT . . . . . . . . . . . . 51

14 W matrix obtained by factorization of fixed length window based STFT 54

$15 \mathrm{~W}$ matrix obtained by factorization of variable length window based

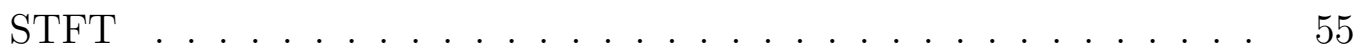

16 a) Fourier transform of MIDI note 79 corresponding to G5, b) Note no 79 derived using fixed length window STFT, and c) Note no 79 derived using variable length window STFT . . . . . . . . . . . 5 56

$17 \mathbf{H}$ obtained on factorization of fixed length window based STFT . . 59 
$18 \mathbf{H}$ obtained on factorization of fixed length window based STFT keeping $\mathbf{W}$ matrix fixed. This $\mathbf{W}$ matrix is available on factorization of variable length window based STFT . . . . . . . . . . . . 60

19 Bar plot of similarity measure of derived notes with ground truth notes using Bhattacharyya distance. . . . . . . . . . . . . . . . 61

20 Artificially generated complex signal. . . . . . . . . . . . . 62

21 Frequency distribution of notes from ground truth table. . . . . . . 63

22 Frequency distribution of notes obtained from factorization of fixed length window STFT. . . . . . . . . . . . . . . . . 64

23 Frequency distribution of the notes obtained from the factorization of the variable length window STFT. . . . . . . . . . . . 65

24 Bar plot of similarity measure of derived notes with ground truth notes using Bhattacharyya distance. . . . . . . . . . . . . . 66

25 Gain vectors of notes obtained from the factorization of the fixed length window STFT. . . . . . . . . . . . . . . . .

26 Gain vectors of notes obtained from the factorization of the variable length window STFT. . . . . . . . . . . . . . . . . 68 


\section{Abbreviations and Acronyms}

\begin{tabular}{|l|l|}
\hline STFT & Short Time Fourier Transform \\
\hline DFT & Discrete Fourier Transform \\
\hline FFT & Fast Fourier Transform \\
\hline NMF & Non-Negative Matrix Factorization \\
\hline MSS & Music Source Separation \\
\hline ICA & Independent Component Analysis \\
\hline MIR & Music Information Retrieval \\
\hline MIDI & Musical Instrument Digital Interface \\
\hline
\end{tabular}




\section{Chapter 1}

\section{Introduction}

\subsection{Motivation}

In this thesis, we are going to concentrate on automatic music note extraction from music recordings. There has been extensive research in music note extraction in the last two decades. Such work concentrates on the assignment of extricating notes from sound sources and gathering them into different sound sources (i.e., instruments). Music note extraction has relevance in the accompanying applications:

1. Music Transcription: Convert audio recording to musical instrument digital interface (MIDI) record structure

2. Music Information Retrieval: Used to investigate and classify music

3. Content Creation: Creating brilliant Karaoke

4. Sound Restoration: Task of removing defects in sound recordings

5. Sound Analysis: Detection of beats, rhythm and meter of music, segmentation, and so on

6. Music Mixing: Creating musical work by controlling existing recordings

7. Capacity: Music is exceedingly repetitive in nature, and consequently by taking in its composition, we can adequately decrease the storage requirements

In this work, we are working with music note extraction for a given piece of recorded music. Commercialization of music and the coming of music based applications on desktop and mobile devices has advanced the exploration in music transcription. Complex musical structures such as beat, meter, rhythm and other qualities 
are obtained by applying some form of reduction on music. The greater part of the music processing community works on reducing complex musical structures to obtain simpler features. Music transcription can be considered as having two sections: i) note extraction and ii) note identification. Note extraction is the task of successfully extracting all the notes played in a music composition. Note identification, also known as note classification, is the task of identifying the note characteristics such as frequency and instrument. In the past, matrix factorization techniques such as the NMF have been increasingly employed to the music source separation problem (MSS). Smaragdis [1] has shown that under certain conditions, the basis vectors from NMF decomposition correspond to music note frequency spectra. The basic NMF technique applied to music note extraction works without any kind of prior information or additional training data. This has motivated us to add to the NMF framework to enhance the quality of note extraction.

\subsection{General Approaches to Note Extraction}

There are many techniques being employed for music note extraction. Some of the popular methods include adaptive signal processing [2], independent component analysis (ICA), sinusoidal modelling, non-negative matrix factorization (NMF) $[1,3-5]$ and probabilistic latent variable models (PLVMs) $[6,7]$.

These techniques can be broadly classified into i) supervised machine learning, ii) unsupervised machine learning, and iii) systems based on signal modelling (informed source separation). On one hand, supervised machine learning algorithms require training data for extraction of notes. A poor training data set will result in poor note extraction. On the other hand, systems based on signal modelling have a low capacity to adapt to signals that do not comply with these models. To overcome this drawback, unsupervised learning approaches have been proposed that use as few hypothesis as possible about the underlying audio content and try to separate the notes blindly. Blind source separation (BSS) techniques are well suited for music note extraction problems. Some of the blind source separation techniques that have been used for music note extraction are i) independent component analysis (ICA),

ii) sparse coding, iii) non-negative matrix factorization (NMF), and iv) non-negative variants of ICA and sparse coding. Non-negative matrix factorization based note extraction techniques are better suited to music note extraction than other methods. 
This is due to the non-negativity constraint imposed by NMF. As a result, the factorization works by identifying the unique events, which are additive in nature. Since music is additive in nature as well, NMF is well suited to address the music note extraction problem.

Non-negative matrix factorization for music separation operates by modelling the magnitude of short time Fourier transform of the recorded signal as a linear combination of prototypical spectral components over time. These prototypical spectral components for each source are learned from the input data and used to estimate the contribution of each source within an unknown mixture and eventually extract notes.

Non-negative matrix factorization has been used in the past as a blind source separation technique in the context of audio $[1,4,5]$ and video processing $[3,8]$, biomedical engineering [9], econometrics [10], and data mining [11]. Non-negative matrix factorization applied to audio signal processing has been used for automatic music transcription, music information retrieval (MIR), audio remixing and so on. A significant amount of research has gone into developing an NMF algorithm for music source separation.

\subsection{Proposed Approach to Note Extraction}

Non-negative matrix factorization (NMF) decomposes a time-frequency representation of a recorded music signal such as the short time Fourier transform (STFT) $\mathbf{X}$ into $\mathbf{W}$ and $\mathbf{H}$ matrices, such that the product of $\mathbf{W}$ and $\mathbf{H}$ matrices, is an approximation of magnitude STFT.

$$
\mathbf{X} \approx \mathbf{W} * \mathbf{H}
$$

The $\mathbf{W}$ matrix consists of the basis vectors learned by the non-negative matrix factorization and the $\mathbf{H}$ matrix details how each of these vectors vary in time throughout the length of the music. These basis vectors contain the frequency spectra of the derived notes. We propose using a variable length window based STFT instead of the traditional fixed length window based STFT for the NMF. The variable length

window based STFT is designed to effectively capture the stationarity of the music signal in a single window.

The improved basis vectors derived using the variable length window based STFT are then used to derive an improved quality gain $(\mathbf{H})$ matrix. 


\subsection{General Approaches to Detect Note Onsets}

A music signal is an event based sound signal. The beginning of a note indicates these events and the procedure of identification of these events is called onset detection, novelty detection or surprise detection. These onset timings are vital for cutting edge music investigation, for example, rhythm examination, beat following, automatic transcription, content delivery, music compression, indexing and music information retrieval (MIR). Onset detection is additionally utilized as a part of real time automatic score followers and enhancing the digital audio workstations with a perspective to event based sound processing.

Onset detection in straightforward music signals (e.g., notes separated in time) can be distinguished as an increment in the energy of a signal. Then again, most real world music signals are complex bits of music with overlapping notes from numerous instruments making it hard to distinguish energy changes in just the time domain signal. To address this issue, detection of energy changes in time-frequency representation has been proposed and widely used in the literature. We can separate the task of onset detection into three stage, specifically, producing a time-frequency representation, calculating spectral distance between time instances and peak selection to choose true onsets from false onsets.

Time-frequency procedures, for example, i) short time Fourier transform (STFT) [12-17]ii) filter banks [18] iii) constant Q-transform [19], and iv) wavelets [20,21] are widely utilized. Most calculations however utilize short time Fourier transform for its simplicity and low computational cost. Well known techniques for detecting spectral changes are i) neural networks (NN) [22], ii) independent component analysis (ICA) $[23,24]$, iii) Kullback-Leibler (KL) divergence [15], iv) Euclidean distance [12-14, 16, 17] and v) adaptive linear prediction [25]. Reduction techniques decrease the two

dimensional time-frequency representation to one dimensional signal. An adaptive thresholding is utilized to choose peaks which relate to note onsets.

\subsection{Proposed Approach to Detect Onset Timings}

The proposed approach uses the short time Fourier transform to generate the timefrequency representation of the signal. We then calculate the spectral distance between time frames using the Itakura-Saito distance and then use adaptive thresholding for selecting appropriate peaks. These peaks are then multiplied with the feature 
sampling rate to obtain the note onset timings.

\subsection{Contributions}

The overall contribution of this thesis is a blind source separation system for music note extraction. Specific contributions are listed as follows:

1. A technique to generate variable length window based short time Fourier transform with each frame effectively capturing the stationarity of the music signal,

2. Evaluation of notes spectra extracted using non-negative matrix factorization from variable length window based short time Fourier transform, showing an improved quality of extracted notes,

3. Evaluation of gain matrix computed using basis vectors obtained using variable length window based short time Fourier transform,

4. Exploration of Itakura-Saito divergence based onset detection to detect note onsets, and

5. Evaluation of Itakura-Saito based onset detection in contrast to other onset detection techniques.

\subsection{Thesis Organization}

In Chapter 2, we begin our discussion by giving a brief overview of the background and foundational information associated with note extraction. In Chapter 3, we discuss our proposed new onset detection technique. Chapter 4, details the new algorithm for music note extraction. Finally in Chapter 5, we discuss our conclusions and future work. 


\section{Chapter 2}

\section{Background and Foundational Information}

\subsection{Structure of Music}

Music can be considered as the addition of notes from a single instrument or multiple instruments at a time. The human ear discriminates between notes by analyzing their frequency spectra. Each note can be distinctly identified by its frequency content and the distribution of this frequency content. The evolution of each note is characterized by i) onset, ii) attack, iii) transient or sometimes decay and iv) offset as illustrated in Fig. 1. The parts of a note are defined as follows

- Onset is the earliest time instance at which a note can be reliably detected. Onset is followed by attack

- Attack is the sudden increase in the gain of the signal

- Transient is the duration between attack and offset (death of the signal). Transient is usually the longest part of a note, and

- Offset is the time instance which marks the death of the signal.

\subsection{Short Time Fourier Transform}

The first stage of note extraction or onset detection is expressing the time domain signal in both time and frequency. A simple Fourier transform of the signal cannot show the time-varying characteristics of the music signal. Hence, we use a timefrequency representation such as the short time Fourier transform (STFT). Short time 


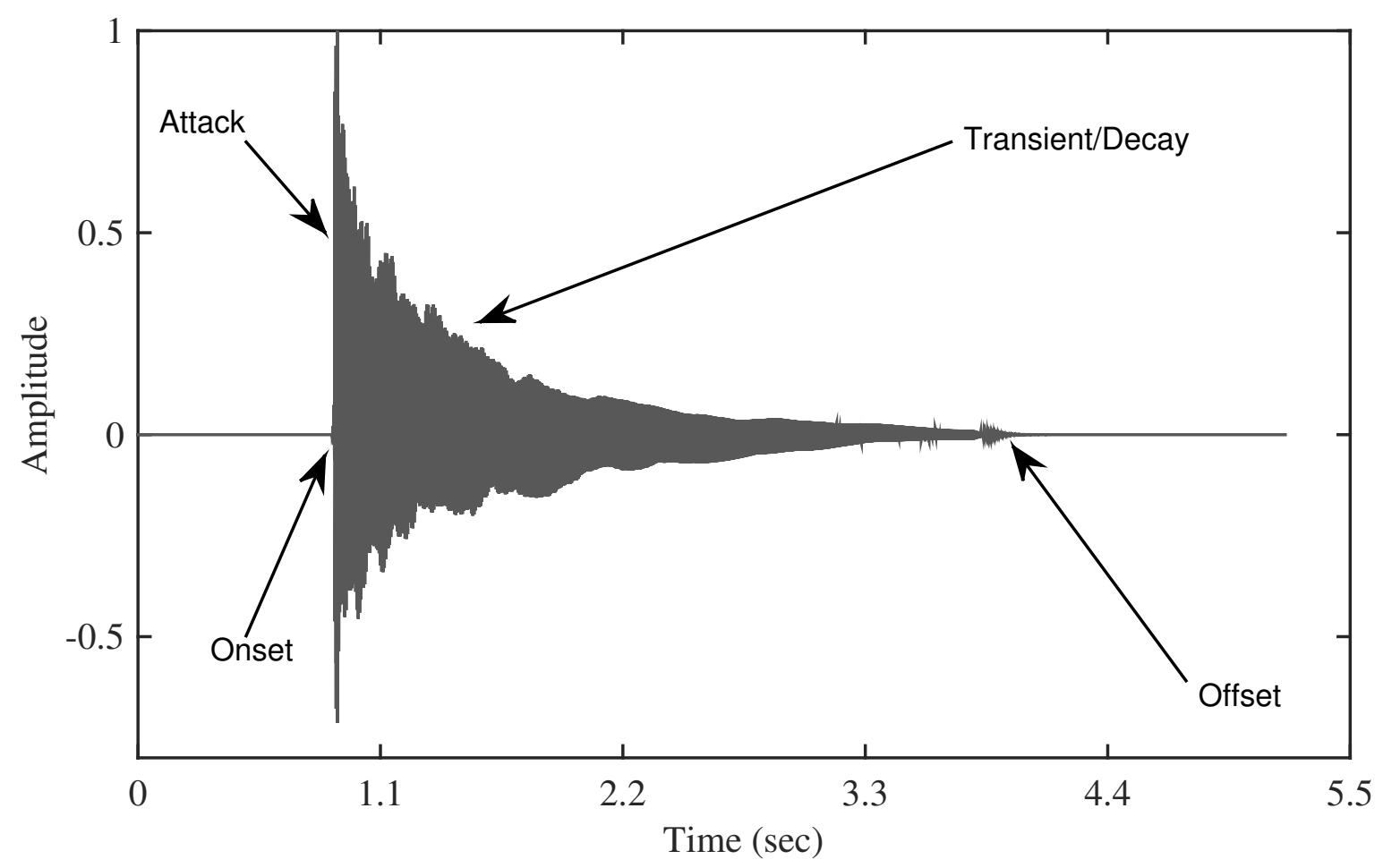

Figure 1: Parts of a note in time domain signal

Fourier transform is a powerful signal processing tool used to analyze the evolution of the signal in both time and frequency. The forward STFT can be thought of as a process to transform a one dimensional signal into a two dimensional signal and the backward or inverse STFT can be thought of as obtaining the time domain signal from the time-frequency representation.

\subsubsection{Discrete Fourier Transform}

The short time Fourier transform is computed by taking the discrete Fourier transform (DFT) over a series of windowed data. The discrete Fourier transform is given as

$$
X_{n}(k)=\sum_{l=0}^{L-1} x_{n}(l) e^{-j \frac{2 \pi k}{L} l} \quad \text { for } \quad k=0,1, \ldots L-1 .
$$


The inverse DFT is given as

$$
x_{n}(l)=\frac{1}{L} \sum_{k=0}^{L-1} X_{n}(k) e^{j \frac{2 \pi k}{L} l} \quad \text { for } \quad l=0,1, \ldots L-1
$$

where $x_{n}(l)$ is the $l^{\text {th }}$ data corresponding to the $n^{\text {th }}$ frame applied to the input time domain signal $x, X_{n}(k)$ is the $k^{\text {th }}$ frequency bin of the $n^{\text {th }}$ frame complex output in the frequency domain, and $L$ is the DFT size in samples. The bandwidth of the frequency bin is given by $2 \pi / L$. The higher the number of input samples, the better is the frequency resolution. The inverse discrete Fourier transform gives us the time domain signal from the complex domain input frequency distribution of the signal. We implement the DFT using a fast Fourier transform (FFT) [26-28] and restrict the input to a real valued signal as we are only concerned with music signals which are real valued. The output of the FFT is a complex domain signal, the real part corresponding to the cosine and imaginary part corresponds to the sine projection of the input signal on a set of sinusoids. Often times, it is of convenience to just consider the magnitude of the this complex valued frequency to estimate the distribution of frequency components present in a signal. The STFT computed with the magnitude of frequency is known as magnitude STFT.

\subsubsection{Forward Short Time Fourier Transform}

The forward STFT or simply STFT is given as

$$
\mathbf{X}(k, m)=\sum_{n=-\infty}^{\infty}\left[x_{n}\right][w(n-m)] e^{-j \frac{2 \pi k}{N} n}
$$

where $x_{n}$ is the time domain signal and $w(n-m)$ is the shifted time domain signal with a window length of $m$ samples. The process of obtaining the STFT is illustrated in Fig. 2. First the time domain signal is segmented by fixed length windows with or without overlap. Typically for music signals, Hamming or Hann windows are used to reduce edge effects. This can also be thought of as a convolution of a time domain signal with sliding windows. The Fourier transform is computed over each window of data. The changing frequency coefficients in time help in understanding the behaviour of the signal in both time and frequency. The window size used in computing the 

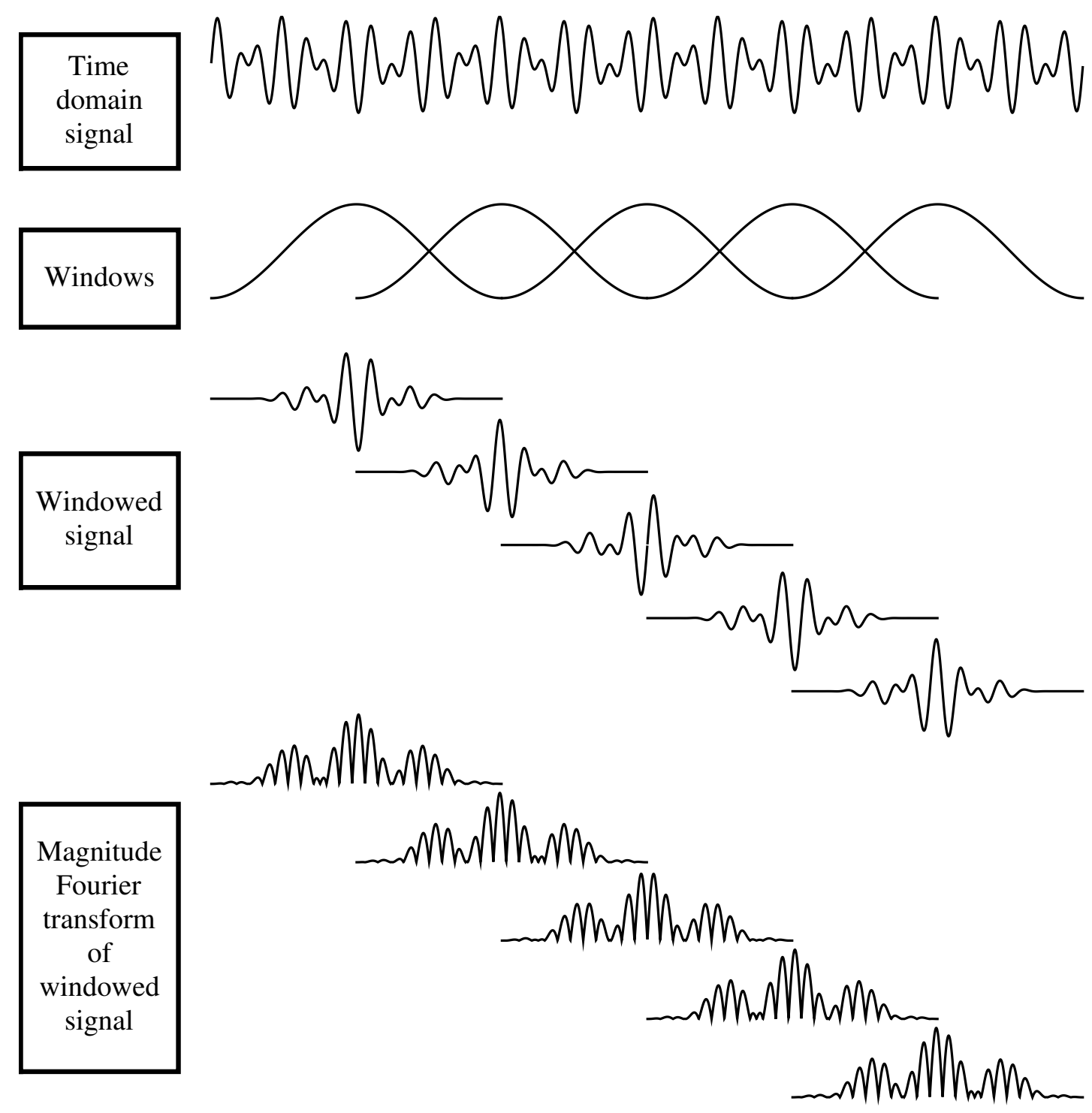

Figure 2: STFT of a signal

STFT influences the temporal and frequency resolution of the representation of the signal. The type of analysis window and the frequency of computing the Fourier transform affects the reconstruction of the signal. Some of these factors that affect the performance are

1. Choice of analysis window, 
2. Analysis window size,

3. Stride, and

4. Fourier transform size

\subsubsection{Choice of Analysis Window and Length}

When the signal is sliced or windowed with a rectangular window, the obtained signal is non periodic. The Fourier transform will interpret the jump at the boundaries as discontinuities and high frequency artifacts are introduced. To avoid these artifacts, various windowing functions have been introduced. These windows are smoothly varying with gain close to 1 at the origin and decaying towards zero at the edges.

Selecting an appropriate analysis window and its size is important as it directly affects the trade off between side lobe attenuation and time resolution. Furthermore, the window function must satisfy certain constraints in order to guarantee that the original signal can be reconstructed from the STFT. Some of the commonly used windows for music signal analysis are Hamming window, Hann window, Bartlett window and Kaiser window. For audio signal processing the Hamming window with an overlap of $50 \%$ of the window length has been found to be most effective for perfect reconstruction using the constant overlap add method (COLA) [29].

For audio signal processing, we want a window function that is symmetric and is a close approximation of a Gaussian curve, with tails approaching zero but not zero in case of reconstruction. To this end, the Hamming window suits our requirements. A Hamming window is given as

$$
\begin{gathered}
\omega(n)=\alpha-\beta \cos \frac{2 \pi n}{N-1}, \\
\text { with } \alpha=0.54, \quad \text { and } \beta=1-\alpha=0.46 .
\end{gathered}
$$

The Fourier transform needs to capture at least one full wave to detect a particular frequency. As a result, a small window will lose significant amounts of lower frequencies, whereas a large window will capture most of the frequencies but will lose the time resolution due to the large length of the window. For music signal with sampling rate of $44.1 \mathrm{kHz}$, a window size of 1024 samples (approximately $23 \mathrm{~ms}$ ) to 4096 samples (approximately $93 \mathrm{~ms}$ ) has been used for analysis based upon the requirement. 


\subsubsection{Choice of FFT Length}

The Fourier transform size influences the frequency bin width. Frequencies detected within a particular bandwidth contribute to a particular bin. The bandwidth of frequency bins is given as

$$
B=f_{\text {sampling }} / N F F T
$$

where $B$ is the bandwidth of the frequency bins in $\mathrm{Hz}, f_{\text {sampling }}$ is the sampling rate of the input signal in $\mathrm{Hz}$ and NFFT is the size or length of the Fourier transform. A large NFFT value gives us small bandwidth allowing for good frequency resolution even at low frequencies and a small NFFT value gives us low frequency resolution at lower frequencies. For a given signal, the value of NFFT should be greater than or equal to the size of the input signal. The given signal is zero padded to accommodate a larger NFFT. In order to take advantage of the computational efficiency of the FFT algorithm, the Fourier transform length is usually chosen to be a power of 2 .

\subsubsection{Choice of Stride}

Stride decides how often the DFT is computed on the signal. A small stride is good for capturing rapid transients, but also results in large data. A large stride is good for analysis of long term evolution of the signal over time and results in relatively less data. The choice of length of stride is based on the application of STFT. Usually overlapped windows are used when reconstruction of signal is necessary. Some of the widely used windowing functions are Hamming window, Hanning window, Blackman window and Kaiser window functions. The amount of overlap between windows impacts the quality of signal reconstruction. The overlap factor for rectangular window is $L / k$ and for Hanning and Hamming window is $((L / 2) / k)$, where $k$ is any positive real value and $L$ is the length of the window. These values give perfect reconstruction when the spectra of the signal is not modified. However, when the spectra of the signal is modified, this overlap factor no longer holds for a good reconstruction. To minimize the aliasing effects, the value of hop size is chosen to exceed the cut-off frequency of the window. For convenience, this cut-off frequency is chosen as the first zero-crossing after the main lobe of the of the window. This gives us an overlap of $50 \%$ for rectangular window and $75 \%$ for Hamming and Hanning windows. Stride is also known as hop size and sometimes is expressed in terms of overlap, such that stride $=$ windowsize - overlap . 


\subsubsection{Time Frequency Resolution of STFT}

At any time instant the STFT gives us the frequency spectra of a window of data. One cannot achieve high frequency resolution with high temporal resolution, i.e., there is a time-frequency trade off. A high frequency resolution tends to give low time resolution, whereas a high time resolution gives a low frequency resolution. This is known as Gabor limit or Heisenberg-Gabor limit. The product of time resolution and frequency resolution is a constant. In practice, the Gabor limit is defined for time-frequency resolution without interference. Higher resolution in both time and frequency can be achieved at the cost of interference.

Short time Fourier transforms obtained with large time resolution are known as wideband STFT and those with large frequency resolution but low time resolution as narrowband STFT. This is illustrated in Fig. 3. We can see that in Fig. 3(a), the wideband STFT has higher temporal resolution but low frequency resolution when compared with the narrowband STFT in Fig. 3(b). Wideband short time Fourier transforms are good for observing the overall evolution of signal and narrowband short time Fourier transforms are good for observing sudden burst or peaks in energy of the signal.

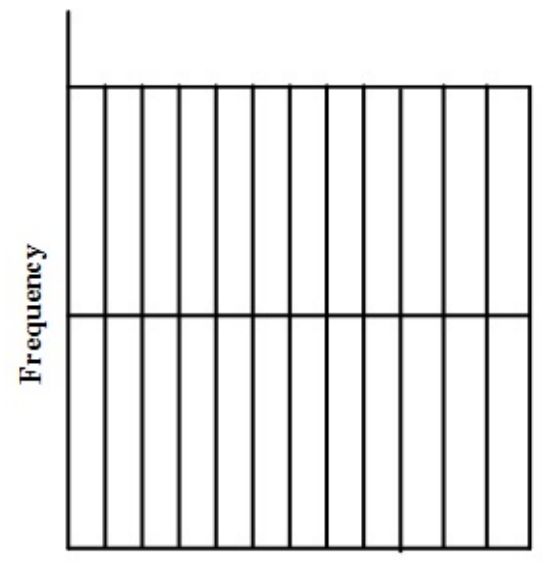

Time

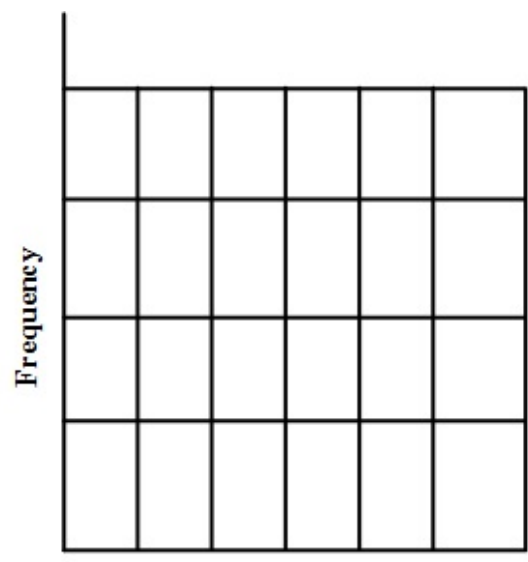

Time

Figure 3: (a) Wideband vs (b) narrowband short time Fourier transform 


\subsection{Introduction to Distance Measures}

There are various methods used to find spectral differences between two time instances. Some of the commonly used spectral distance measures are a) squared Euclidean distance [12], b) variants of squared Euclidean distance [13, 14, 16, 17, 19], c) modified Kullback-Leibler distance [15], and $d$ ) Itakura-Saito distance. [30]

The Euclidean distance is defined as the distance between two vectors $P$ and $Q$ of length $n$. It can also be thought of as a distance between two points in $n$ dimensional space. The Euclidean distance between $P$ and $Q$ is given as

$$
D_{E U}(P, Q)=\sqrt{\sum_{i}(Q(i)-P(i))^{2}} .
$$

The Kullback-Leibler (KL) divergence is used to measure the information loss between two probability distributions $P$ and $Q$, when $Q$ is used to approximate $P$, given as

$$
D_{K L}(P \| Q)=\sum_{i} P(i) \cdot \log \frac{P(i)}{Q(i)} .
$$

The Kullback-Leibler divergence is also known as information gain, information divergence or relative entropy. It is non-symmetric in nature.

The Itakura-Saito (IS) distance [30] was originally developed as part of the error measure in pioneering work on linear predictive coding of speech. In speech signal processing, it is used to measure the dissimilarity of the power spectra of a frame of clean speech and synthesized speech. The Itakura-Saito distance is given as

$$
D_{I S}(P \| Q)=\sum_{i} \frac{|P(i)|}{|Q(i)|}-\ln \frac{|P(i)|}{|Q(i)|}-1
$$

where $Q$ is a spectrum to compare with $P$. Note that the Itakura-Saito distance measure is a Bergman divergence and is non symmetric which means it does not satisfy the triangle inequality; also, the Itakura-Saito distance is a special case of the $\beta$-divergence for $\beta=0$. [31]

For every exponential family probability distribution, there exists a corresponding generalized distance measure. This is shown in Table 1.

From Figure 1, it is easy to see that a typical note in music is approximately an 
Table 1: Distribution and distance measures

\begin{tabular}{|l|l|}
\hline Distribution & Distance Measure \\
\hline Spherical Gaussian & Squared Euclidean distance \\
\hline Multinomial & Kullback-Leibler distance \\
\hline Exponential & Itakura-Saito distance \\
\hline
\end{tabular}

exponential distribution with a positive exponential increase in amplitude between the onset and attack followed by constant gain or decreasing gain. Since, we are interested in detecting the onsets of notes, we largely focus on the signal between the onset and attack.

\subsection{Onset Detection Function}

\subsubsection{Scheme of Onset Detection}

The method of onset detection is illustrated in Fig. 4 and Fig. 5. The process of onset detection has three steps i) pre-processing, ii) reduction, and iii) post-processing . These stages are described in Fig. 4. The pre-processing stage consists of converting the time domain signal to time-frequency representation such as the STFT. The time domain signal in Fig. 5(a) is used to get the STFT illustrated in Fig. 5(b). The pre-processing stage is used to develop signal features which make it easier to detect spectral changes in the signal. Then comes the reduction stage. This is the most important stage of onset detection. The given input audio, after pre-processing is then reduced to obtain a one dimensional signal. Fig. 5(c) represents the detection function obtained after reduction of the time-frequency transformation of the signal. This reduced signal has more information regarding the onsets and tries to reduce or eliminate other information not relevant to onset detection. This is where most of the algorithms differ and an efficient reduction technique gives better onset detection. The post-processing stage consists of using a peak picking strategy to select the appropriate peaks. These selected peaks correspond to onset timings in the given music file. The onsets detected are represented as asterisks in Fig. 5(d). 


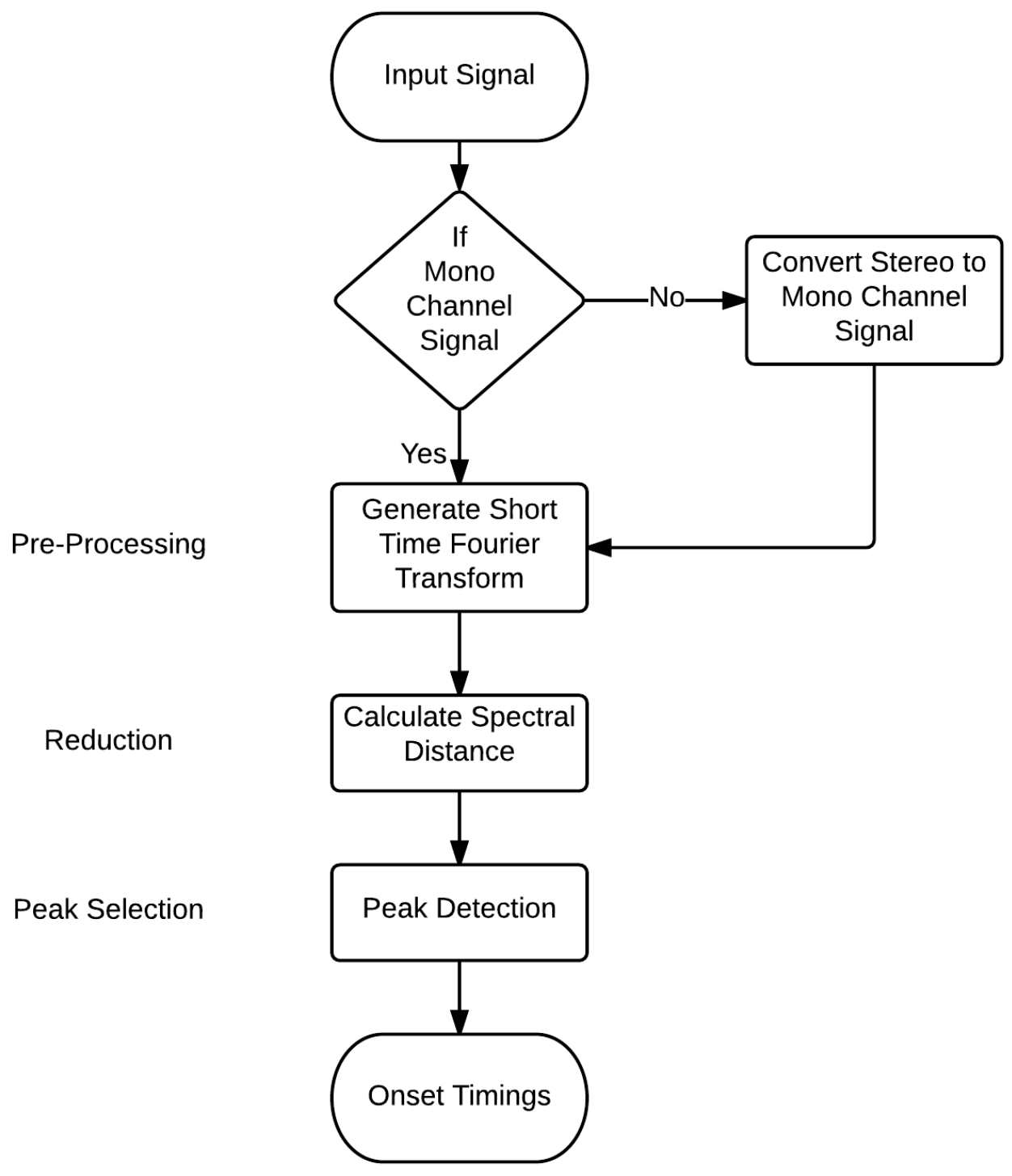

Figure 4: Onset detection flowchart

\subsubsection{Pre-processing}

Audio signals are additive in nature, i.e., notes from different instruments superimpose and do not conceal each other. It is quite simple to detect note onsets when two notes are separated in time. However things get complicated with overlapping notes from different instruments. Thus, it makes sense to analyze information across various frequency bands. Several onset detection techniques use some form of preprocessing 


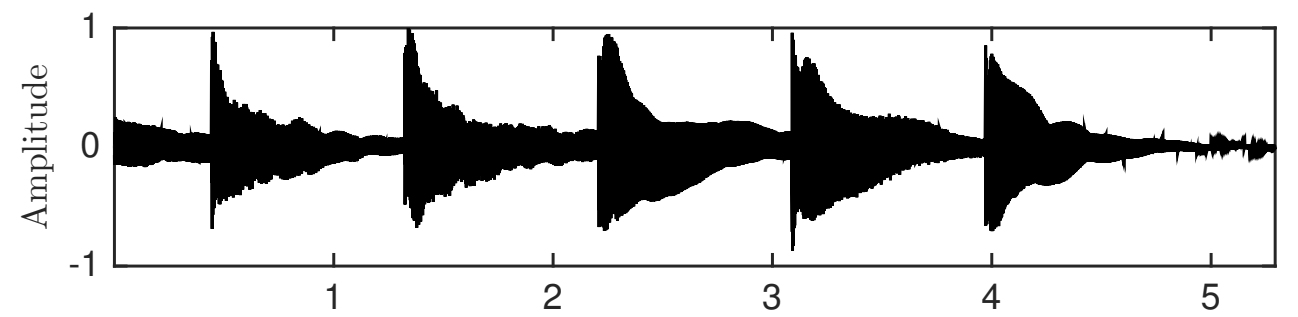

(a)

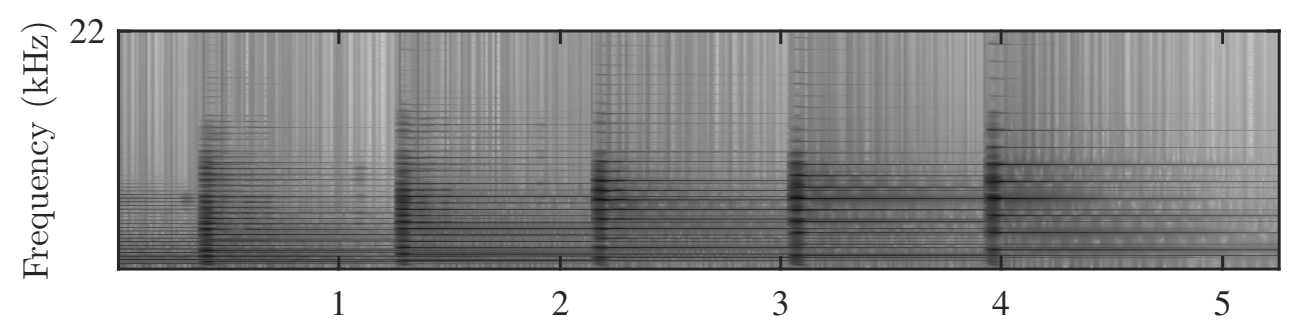

(b)

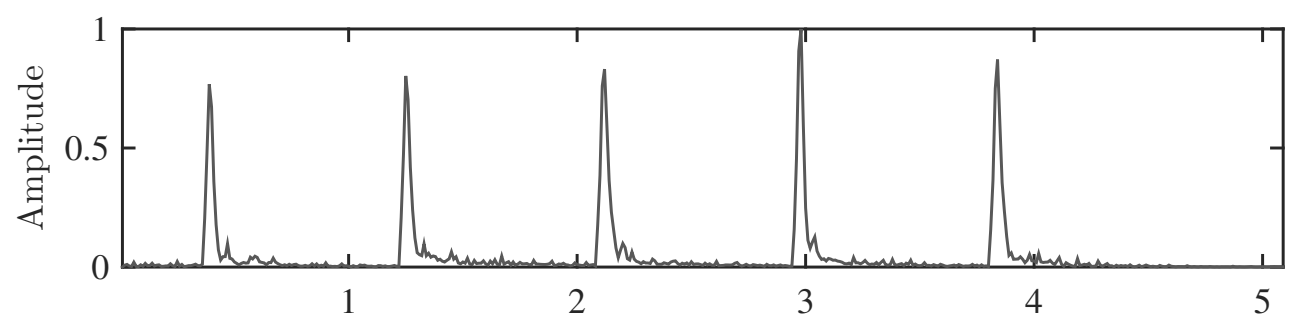

(c)

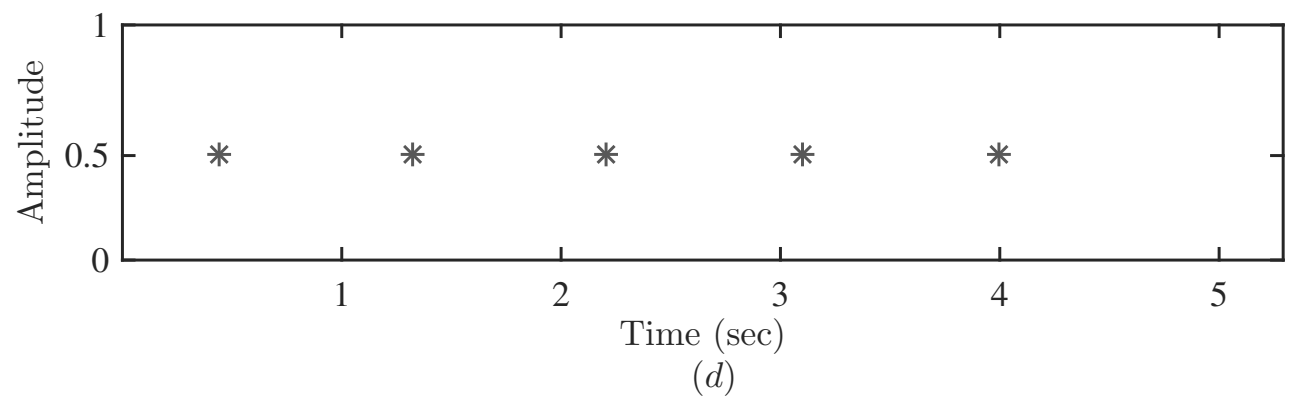

Figure 5: Scheme of onset detection depicting (a) time domain signal (b) short time Fourier transform of the time domain signal (c) detection function for note onsets, and (d) note onsets detected

to split the signal into various frequency bands to analyze information independently. Goto [32] slices the spectrogram into spectrum strips and recognizes onsets by detecting sudden changes in energy in each spectrum strip. Scheirer [33] implements a 
six-band filter bank, using sixth order elliptic filters, and psycho-acoustically inspired processing to produce onset trains. Klapuri [34] uses a filter bank that divides the signal into 8 non-overlapping bands. Onsets are detected in each band and finally combined. Duxbury et al. [16] use constant Q conjugate quadrature filter bank to separate the signal to five sub-bands. By implementing a multiple band scheme the approach effectively avoids the constraints imposed by the use of a single reduction method, while having different time resolutions for different frequency bands.

Other methods such as signal modelling have also been used to produce modified signals which have been used to detect onsets. Spectral modelling synthesis (SMS) [35] explicitly considers the residual of the synthesis method as a Gaussian white noise filtered with a slowly varying low-order filter. Levine [36] calculates the residue between original signal and synthesized signal. Increase in residual energy marks the onsets. Giaconi et al. [37] use discrete wavelet transform to extract the wavelet packet energy coefficients which they use along with other audio spectral features to train a bidirectional long short term memory recurrent neural network to detect note onsets. Many methods have been used to detect onsets, from which the most popular methods have been covered.

\subsubsection{Reduction}

The preprocessed audio thus obtained is then reduced to get the detection function. The reduction process depends upon pre-processing technique. The reduction technique is most instrumental in reducing the pre-processed audio signal to obtain a detection function. Some of the widely used reduction techniques are described below.

\section{Energy Flux by Laroche}

Energy flux [12] is calculated by computing short time Fourier transform frames using a window size of 2048 samples and hop size of 512 samples, for a sampling rate of $44.1 \mathrm{kHz}$, resulting in an input feature resolution of $11.6 \mathrm{msec}$. Each input feature sample $E F(n)$ is calculated as magnitude of differences of root mean square (RMS) value between the current short time Fourier transform and its predecessor using

$$
E F(n)=\left|R M S\left(X_{n}(k)\right)-R M S\left(X_{n-1}(k)\right)\right|
$$


where $X_{n}(k)$ is the $k_{t h}$ frequency bin of $n_{t h}$ frame of STFT.

\section{Spectral Flux by Paul Masri}

Spectral flux [13] is calculated by computing the short time Fourier transform frames of window size 2048 samples and hop size of 512 samples for a sampling rate of 44.1 $\mathrm{kHz}$, resulting in an input feature resolution of 11.6 msec. Each input feature sample $S F(n)$ is calculated as the sum of the positive differences in magnitude between each frequency bin of the current short time Fourier transform frame and its predecessor using

$$
S F(n)=\sum_{k=1}^{K} H\left(\left|X_{n}(k)\right|-\left|X_{n-1}(k)\right|\right)
$$

where $H$ denotes the Half wave rectification process.

\section{Spectral Flux Log Filtered by S Böck}

In spectral flux log filtered [19], Bock et al. propose filtering the magnitude of short time Fourier transform with a pseudo Constant $Q$ filter bank, where the frequencies are aligned to the frequencies of semitones ranging from $27.5 \mathrm{~Hz}$ to $16 \mathrm{kHz}$, using a short time Fourier transform of window size of 2048 samples and hop size of 512 samples. The resulting filter bank, $F(k, b)$, has $\mathrm{B}=82$ frequency bins with $b$ denoting the frequency bin and $k$ denoting the bin number of linear spectrogram. The filter bank is not normalized resulting in emphasis of higher frequency bins, similar to the HFC method [13]. Each input feature sample is calculated as the sum of the positive differences in log magnitude between each frequency bin of the current short time Fourier transform and its predecessor using

$$
\operatorname{SFLF}(n)=\sum_{b=1}^{B=82} H\left(\left|X_{n}^{\log f i l t}(b)\right|-\left|X_{n-1}^{\log f i l t}(b)\right|\right)
$$

where,

$$
X_{n}^{\log f i l t}(b)=\log \left(\lambda \cdot\left(\left|X_{n}(k)\right| \cdot F(k, b)\right)+1\right)
$$

with compression parameter of $\lambda=20$. 


\section{Complex Spectral Difference by J. Pablo et. al.}

Complex spectral difference [14] is calculated from short time Fourier transform of window size of 1024 samples and hop size of 512 samples, resulting in a feature resolution of $11.6 \mathrm{~ms}$ for music sampled at $44.1 \mathrm{kHz}$. $C S D(n)$ produces large value if there is a significant change in amplitude from the expected phase value. Unlike in the spectral flux, the complex spectral flux density takes into account the phase information as well and is computed using

$$
C S D(n)=\sum_{k=1}^{K}\left|X_{n}(k)-\hat{X}_{n}(k)\right|
$$

\section{Modified Kullback-Leibler by Hainsworth and MacLeod}

Modified Kullback-Leibler (MKL) presented by Hainsworth and MacLeod [15] is a harmonic change detection calculated by computing a short time Fourier transform using a window size of 2048 samples and hop size of 512 samples, for music sampled at $44.1 \mathrm{kHz}$. MKL uses a modified Kullback-Leibler distance measure to detect spectral change between frequency bins of consecutive frames. The modified measure is thus tailored to accentuate positive energy change. The modified Kullback-Leibler is computed using

$$
M K L(n)=\sum_{k=1}^{K} \log _{2} \frac{\left|X_{n}(b)\right|}{\left|X_{n-1}(b)\right|} .
$$

\section{Spectral Difference by C. Duxbury et al.}

Duxbury [16] uses the L2 norm instead of the L1 norm used by Paul Masri [13] on a short time Fourier transform computed with a window size of 2048 samples and hop size of 512 samples on music sampled at $44.1 \mathrm{kHz}$ resulting in a feature sampling rate of $11.6 \mathrm{~ms}$. The spectral difference is computed as

$$
S D(n)=\sum_{k=1}^{K} H\left(\left|X_{n}(k)\right|-\left|X_{n-1}(k)\right|\right)^{2} .
$$

\section{Harmonic Feature Content by Paul Masri}

Harmonic feature content (HFC) [13] is harmonic change detection function, calculated by computing the short time Fourier transform with a window size of 2048 and 
a hop size of 512 samples. HFC weighs the magnitude spectrogram by bin number to emphasize higher bin frequencies and is computes as

$$
H F C(n)=\frac{1}{N} \sum_{k=1}^{K} W_{k}\left|X_{k}(n)\right|^{2} .
$$

\section{Rectified Complex Domain by Simon Dixon}

Complex spectral difference [17] takes into consideration both positive and negative energy difference between consecutive time frames. This means both onsets and offsets are taken into consideration while detecting onsets. This is rectified by taking the half wave rectification to detect positive increase in energy and is given by

$$
R C D(n)=\sum_{k=1}^{K} H\left(X_{n}(k)-\hat{X}_{n}(k)\right)
$$

\subsubsection{Peak Selection}

To extract the appropriate onset timings from the onset detection function, peak selection methods are used that typically include post-processing, thresholding and peak-picking.

Post processing is an optional step. It is used to make it easy to pick peaks from the onset detection function. This usually includes the use of normalization and/or filters. The normalization works in one of two ways [17,38] (i) subtract the average and divide by the maximum value, so that the function will be in the interval of $[-1,1]$; (ii) subtract the average from each value and divide by absolute deviation, so that the function will be between interval $[0,1]$. Sometimes low pass filters are also used, which helps in reducing content due to the transient part of the note.

Two types of thresholding methods are available: (i) constant thresholding and (ii) adaptive thresholding. Due to the great dynamics common in music signals, a constant thresholding will give weak results [39]. Adaptive thresholding is known to provide much better results $[17,38,39]$. Adaptive thresholding based on mean or median are usually used in the literature for onset detection, given as

$$
\begin{gathered}
\hat{\delta}(n)=\delta+\lambda \operatorname{mean}(|d(n-M)|, \ldots,|d(n+M)|), \text { and } \\
\hat{\delta}(n)=\delta+\lambda \operatorname{median}(|d(n-M)|, \ldots,|d(n+M)|)
\end{gathered}
$$


where $\lambda$ and $\delta$ are positive constants, that can be tweaked and $(2 M+1)$ is the size of the window about each feature in the onset detection function $d . \hat{\delta}(n)$ is the calculated adaptive threshold value at time instance $n$.

Every feature in the onset detection function is compared with its corresponding adaptive threshold value and if greater is selected as an onset. An additional criteria is sometimes also used, which requires the onset to be a local maximum in a window of length $2 w[17,22]$.

$$
o(n)= \begin{cases}1 & \text { if } d(n)>\hat{\delta}(n) \\ & \text { and } d(n-w) \leq d(n) \leq d(n+w) \\ 0 & \text { otherwise }\end{cases}
$$

\subsection{NMF}

Non-negative matrix factorization (NMF) is a data-driven machine learning technique [40]. NMF methods can also be thought of as basis decomposition or dictionary-based method and is closely related to sparse coding [41,42], principal component analysis [43], singular value decomposition [44], independent subspace analysis methods [4547] and related matrix factorization methods. In addition to audio applications, NMF is also commonly used for processing images, text, and other data types and collectively have gained a significant research interest over the past decade.

The goal of NMF is to approximate a non-negative $M \times N$ matrix $\mathbf{X} \in \mathbb{R}_{\geq 0}^{M \times N}$ as a product of two non-negative matrices $\mathbf{W} \in \mathbb{R}_{\geq 0}^{M \times K}$ and $\mathbf{H} \in \mathbb{R}_{\geq 0}^{K \times N}$, such that the error of reconstruction is minimized. That is, NMF aims to decompose $\mathbf{X}$ as

$$
\mathrm{X} \approx \mathbf{W H}
$$

usually under some $p$-norm minimizing cost function

$$
\underset{\mathbf{W}, \mathbf{H}}{\operatorname{argmin}} F(\mathbf{W}, \mathbf{H})=\|\mathbf{X}-\mathbf{W H}\|_{p}
$$

and often with additional constraints or regularization terms.

At a high level, when we use NMF for audio source separation, we decompose the audio short time Fourier transform data, or equivalently the magnitude of the short-time Fourier transform (STFT) of an audio recording, as a linear combination 
of the outer product of prototypical spectral components times vectors of amplitude over time. The spectral components for each sound source and their gains are learned from data and the result is used to estimate the contribution of each source within an unknown mixture over time, and eventually perform separation.

Some of the commonly used update algorithms for $\mathbf{W}$ and $\mathbf{H}$ are multiplicative update algorithm, gradient descent and alternating least squares method. The multiplicative update rules are given as

$$
\begin{gathered}
\mathbf{H}_{b j}^{q+1}=\mathbf{H}_{b j}^{q} \frac{\left(\left(\mathbf{W}^{q+1}\right)^{T} \mathbf{X}\right)_{b j}}{\left(\left(\mathbf{W}^{q+1}\right)^{T} \mathbf{W}^{q+1} \mathbf{H}^{q}\right)_{b j}}, \forall j, b \\
\mathbf{W}_{i a}^{q+1}=\mathbf{W}_{i a}^{q} \frac{\left(\mathbf{X}\left(\mathbf{H}^{q}\right)^{T}\right)_{i a}}{\left(\mathbf{W}^{q} \mathbf{H}^{q}\left(\mathbf{H}^{q}\right)^{T}\right)_{i a}}, \forall i, a
\end{gathered}
$$

Equation 25 and 26 are updated iteratively until the error between $\mathbf{X}$ and the product of $\mathbf{W}$ and $\mathbf{H}$ is lower than the set limit or the number of iterations reaches the maximum iteration limit.

The gradient descent algorithm rules are given as

$$
\mathbf{H}^{q+1}=\max \left(0, \mathbf{H}^{q}-\alpha_{q} \Delta_{H} \mathcal{F}\left(\mathbf{W}^{q}, \mathbf{H}^{q}\right)\right),
$$

and

$$
\mathbf{W}^{q+1}=\max \left(0, \mathbf{W}^{q}-\alpha_{q} \Delta_{W} \mathcal{F}\left(\mathbf{W}^{q}, \mathbf{H}^{q+1}\right)\right),
$$

where $\alpha_{q}$ is the fixed size step and $\Delta_{H} \mathcal{F}\left(\mathbf{W}^{q}, \mathbf{H}^{q}\right)$ and $\Delta_{W} \mathcal{F}\left(\mathbf{W}^{q}, \mathbf{H}^{q+1}\right)$ are gradients of cost function with respect to $\mathbf{H}$ and $\mathbf{W}$ respectively. Equation 27 and 28 are updated iteratively until the error between $\mathbf{X}$ and the product of $\mathbf{W}$ and $\mathbf{H}$ is lower than the set limit or the number of iterations reaches the maximum iteration limit.

The alternating least squares algorithm has the following update rules,

$$
\mathbf{H}^{q+1}=\underset{W \geq 0}{\operatorname{argmin}} \mathcal{F}\left(\mathbf{W}^{q+1}, \mathbf{H}^{q}\right),
$$

and

$$
\mathbf{W}^{q+1}=\underset{H \geq 0}{\operatorname{argmin}} \mathcal{F}\left(\mathbf{W}^{q}, \mathbf{H}^{q+1}\right) .
$$

Equation 29 and 30 are updated iteratively until the error between $\mathrm{X}$ and the product of $\mathbf{W}$ and $\mathbf{H}$ is lower than the set limit or the number of iterations reaches the maximum iteration limit. Alternating least squares is shown to converge faster than 
the multiplicative update rules [48].

In non-negative matrix factorization $(\mathrm{NMF})$, the non-negativity of the matrices involved fit the only constraint used to process the decomposition. The approximation comes from the constraint $R<\operatorname{minimum}(N, M)$, so that the factorization is also a rank reduction.

\subsubsection{NMF on Music}

For music separation using NMF, we normally use a non-negative time-frequency representation of the music for $\mathbf{X}$. For general matrix factorization the matrix $\mathbf{X}$ does not need to be non-negative, but having a non-negative matrix results in efficient algorithms for finding the factorization using only additive components [3,49]. NMF is essentially a parts based learning method. Since, music can be thought of as an addition of notes in time, NMF is very well suited for the task of note extraction. Other blind source separation techniques such as the principal component analysis (PCA) [43] and independent component analysis (ICA) [45] use both addition and subtraction to find the basis vectors. As a result, they do note extract parts as NMF does.

The most commonly used non-negative time-frequency representation used for music separation is the magnitude of the short-time Fourier transform (STFT). Given that $\mathrm{X}$ is $M \times N$, the usual procedure for NMF is to start with segmenting the audio signal into $N$ windows, each of length $M$. Non-negative matrix factorization can decompose the original signal into the sum of different basis. Smaragdis [1] showed that the components in basis matrix $\mathbf{W}$ can be individual notes and proposed an approach of polyphonic music transcription using NMF. If the STFT is the timefrequency representative of choice, then an $M$-point fast Fourier transform (FFT) is typically applied to each segment of windowed data, resulting in the $M \times N$ matrix $\mathbf{X}$. Zero-padding before the FFT could also be used such that the windowing is done with some length $P<M$ and then zero-padded by $M-P$ zeros to a final length of $M$. Therefore, with a time-domain music signal $x(t)$, we normally compute

$$
X(t, \omega)=\operatorname{STFT}\{s(\tau) \gamma(\tau-t)\}
$$

where $\gamma(\tau-t)$ is a small time window. Then $\mathbf{X}$ is obtained by taking the magnitude STFT as $\mathbf{X}=|X(t, \omega)|$ for an $M \times N$ matrix. Given a matrix $\mathbf{X}$, the NMF decomposes 


\section{Matrix (STFT)}
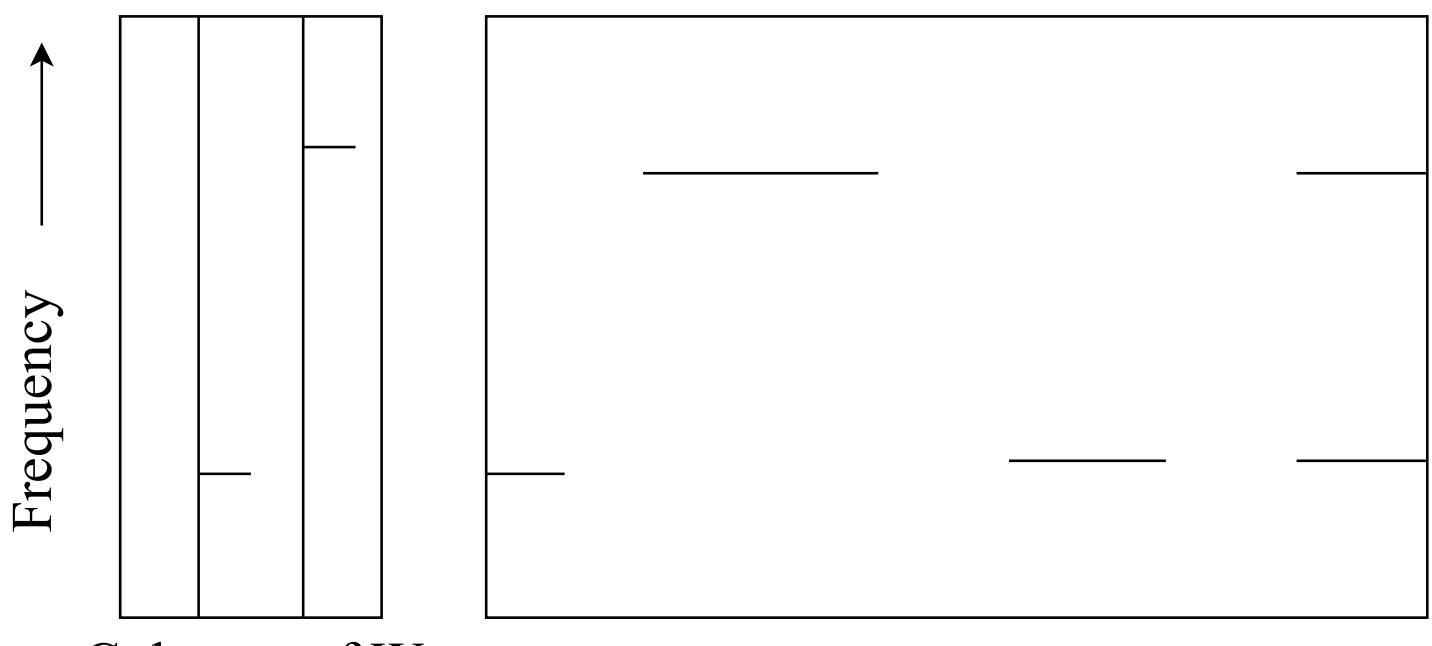

Columns of $\mathrm{W}$

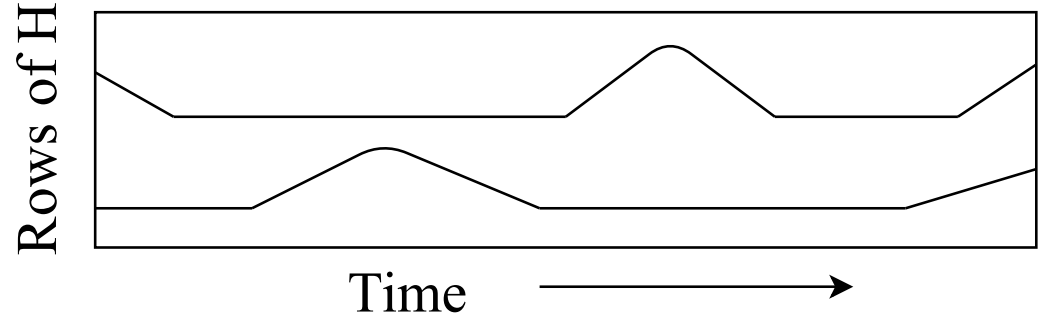

Figure 6: Example of NMF decomposition

it into matrices $\mathbf{W}$ and $\mathbf{H}$ as illustrated in Fig. 6. The basis vectors (i.e., columns) in $\mathbf{W}$ matrix can be considered as a representation of the harmonic content of a note in $\mathbf{X}$ and the corresponding rows in $\mathbf{H}$ matrix depict the notes evolution in time. Note that matrix $\mathbf{H}$ also shows note profiles that would be represented by varying intensities in matrix $\mathbf{X}$. From Fig. 6 we can see that the columns in $\mathbf{W}$ contain the basis vectors and the rows in $\mathbf{H}$ contain the gain of these basis vectors in time. 


\section{Chapter 3}

\section{Onset Detection Function}

\subsection{Methodology/Algorithm}

In this chapter, we discuss in detail the algorithm developed for extracting onset timings from a given piece of recorded music. The outline of the algorithm is as described in Fig. 7. For simplicity, the algorithm is split into three major steps:

1. Pre-processing

2. Reduction

3. Post-processing

Let us understand each step in detail.

\subsubsection{Pre-processing}

In this step, we are converting the given piece of recorded music into a time-frequency representation, which will be used as an input in the reduction step. Most of the recorded music are available in stereo format. Also, music produced in a studio is edited acoustically, as a result of which an instrument present in one channel may not be present in another. In order to check the accuracy of the algorithm, we deal with mono-channel audio instead of stereo. To obtain the mono-channel audio signal, we take the average of the signal over all the channels to get a representation of the signal. This provides a good estimate of the frequencies present in all the channels at any instant of time and also reduces the computation required by a factor of $1 / c$, where $c$ is the number of channels. The mono-channel audio signal thus obtained is 


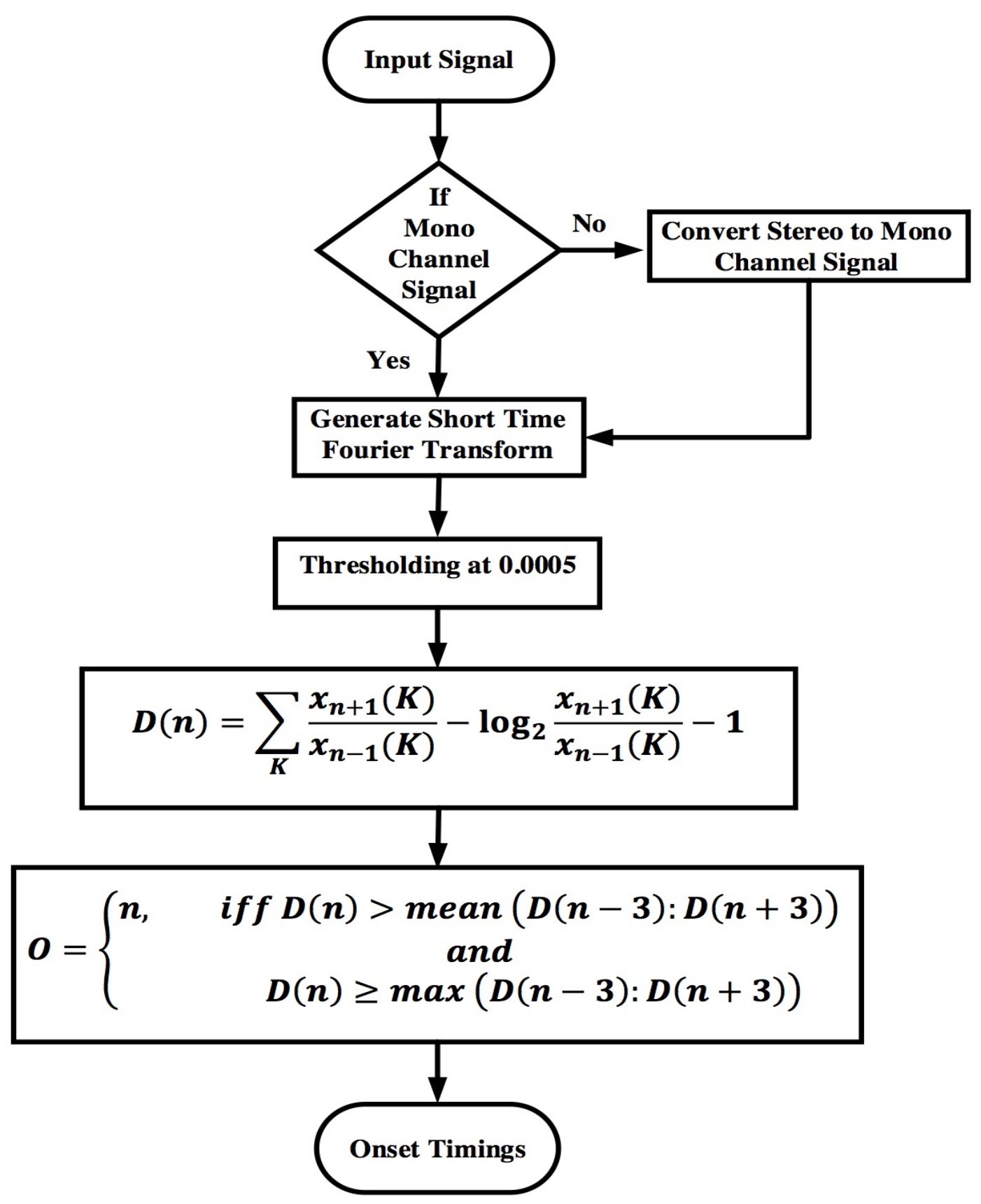

Figure 7: Onset detection flowchart 
now used to generate the short time Fourier transform (STFT) of the given music recording.

It is important to choose correct parameters for the STFT so that a good timefrequency resolution trade-off is obtained. We choose a window size of 2048 samples with a hop size of 512 (75\% overlap) resulting in a feature resolution of $11.6 \mathrm{~ms}$. This STFT employs a fast Fourier transform (FFT) of 2048 samples.

For music sampled at $44.1 \mathrm{kHz}, 2048$ sample size FFT will detect a minimum frequency of $21.53 \mathrm{~Hz}$ with a frequency bin bandwidth of $21.53 \mathrm{~Hz}$. Fourier transform of 2048 samples can detect the lowest key of the piano at $27.5 \mathrm{~Hz}$. Hence, we choose the Fourier transform size equal to 2048 samples, as it is small enough to reduce time uncertainty and still have good frequency resolution. Any frequency component below the minimum frequency along with noise contributes to spectral leakage. This spectral leakage is reduced by thresholding the normalized STFT. This value is empirically determined to be 0.0005 .

\subsubsection{Reduction}

The STFT obtained after thresholding contains frequency content corresponding to the attack, transient and offset. We would like to reduce the attack, transient and offset, thereby leaving only the onset information pertaining to a note. This is accomplished by using various distance measures between two adjacent frames of the STFT. In the literature, the Euclidean distance, Kullback-Leibler divergence and their variants have been widely used. We apply a new distance measure derived from the Itakura-Saito divergence. We believe the Itakura-Saito divergence will perform better as compared to the Euclidean and Kullback-Leibler distance measures and is well suited for this kind of data processing. The Itakura-Saito divergence is given as

$$
D_{I S}(n)=\sum_{K} \frac{\left|\mathbf{X}_{p}(k)\right|}{\left|\hat{\mathbf{X}}_{p}(k)\right|}-\ln \frac{\left|\mathbf{X}_{p}(k)\right|}{\left|\hat{\mathbf{X}}_{p}(k)\right|}-1, \quad k=1 \ldots K
$$

where $\mathbf{X}_{p}(k)$ is the spectrum of a signal to compare against it's de-noised version $\hat{\mathbf{X}}_{p}(k) . K$ is the total number of elements in $X_{p}$. We are using a slightly modified version of the Itakura-Saito divergence given as

$$
D_{I S}(n)=\sum_{K} \frac{\left|\mathbf{X}_{p}(k)\right|}{\left|\mathbf{X}_{q}(k)\right|}-\log _{2} \frac{\left|\mathbf{X}_{p}(k)\right|}{\left|\mathbf{X}_{q}(k)\right|}-1, \quad k=1 \ldots K
$$


where $\mathbf{X}_{p}(k)$ denotes the $k^{t h}$ frequency bin of $p^{t h}$ frame of STFT $\mathbf{X}$ and $\mathbf{X}_{q}(k)$ denotes the spectrum at $q^{\text {th }}$ frame that we want to compare for onset detection at time instance $n$. In the rest of this thesis, we will call our method the Itakura-Saito, for the sake of simplicity. We choose $\mathbf{X}_{p}(k)=\mathbf{X}_{n+1}(k)$ and $\mathbf{X}_{q}(k)=\mathbf{X}_{n-1}(k)$. For $\mathbf{X}_{n+1}(k)=\mathbf{X}_{n-1}(k)$, the Itakura-Saito divergence $\left(D_{I S}\right)$ gives zero. For increasing divergence between the two frames, $D_{I S}$ increases. The Itakura-Saito divergence gives us a scalar measure between two time frames. This way, we convert two dimensional time-frequency information to one dimensional detection function which we use as an onset detection function. A note onset is characterized by a peak in the detection function. The signal obtained after reduction with the Itakura-Saito divergence is then thresholded to pick peaks corresponding to the note onsets. This is done in the next section.

\subsubsection{Post-Processing}

Selection of peaks from the detection function is known as peak-picking. A number of peak picking algorithms are available in the literature. Most of these peak picking strategies use some kind of thresholding. A hard thresholding will pick strong onsets and ignore weak onsets. Hence, an adaptive thresholding with a moving mean window is widely used. Eq.(34) describes the peak picking strategy we use for Itakura-Saito based onset detection.

$$
O= \begin{cases}1, \quad & \text { iff } D_{I S}(n)=\max \left(D_{I S}(n-3) \ldots D_{I S}(n+3)\right) \\ \& & \\ & D_{I S}(n) \geq \operatorname{mean}\left(D_{I S}(n-3) \ldots D_{I S}(n+3)\right) \\ 0, & \text { otherwise }\end{cases}
$$

The peaks so obtained correspond to detected onsets.

\subsection{Experimental Setup and Results}

All the experiments are run under MacOS on a MacBook Pro with 2.6 GHz Intel i7 processor and $16 \mathrm{~GB} 1600 \mathrm{MHz}$ DDR3 RAM. All the test scripts are written using the DSP toolbox in MATlab 2014b. For the experiments, we use a database of 8 single instrument MIDI files and one multiple instrument MIDI file. Audio files 
are generated using MIDI files in Garageband 10 with a sampling frequency of 44.1 $\mathrm{kHz}$. Any stereo channel recordings are first converted to mono channel before any further processing. To generate the STFT, the spectrogram function from the signal processing toolbox in MATLAB is used.

Along with the proposed Itakura-Saito method (IS) and weighted Itakura-Saito method, 5 other popular methods are used to compare against the proposed approach: high frequency component (HFC) [13], spectral difference (SD) [16], spectral flux (SF) [13], complex spectral difference (CD) [50], and rectified complex domain (RCD) [17]. The ground truth table is obtained from the MIDI files in MATLAB using the MIDI toolbox [51].

For analyzing the music signals, any stereo information is first converted through simple averaging to form a mono-channel. For each of the algorithms requiring a timefrequency representation, a spectrogram from an STFT is used with window size of 2048 samples and hop size of 512 samples giving a feature sampling rate of $11.2 \mathrm{~ms}$ for a sampling rate of $44.1 \mathrm{kHz}$. An FFT length for each rectangularly windowed segment is set to 4096 samples. Then each algorithm is performed, including the proposed technique to perform the onset detection. The extracted onset timings are compared with values in ground truth table extracted from the original MIDI file within a window of $\pm 25 \mathrm{~ms}$ or a window of $50 \mathrm{~ms}$ about a true onset.

We use the following measures to rank the proposed method in comparison to other methods.

- True Positive (TP): count of onsets detected that are present in the ground truth table

- False Positive (FP): count of onsets detected not present in the ground truth table

- False Negative (FN): count of onsets present in ground truth table but not present in the list of detected onsets

- Accuracy (Acc): general measure of relevant onsets detected to the total number of onsets

$$
A c c=\frac{T P}{T P+F P+F N}
$$

- Precision $(\mathrm{P})$ : ratio of relevant returned onsets to the total number of returned 
onsets

$$
P=\frac{T P}{T P+F P}
$$

- Recall (R): ratio of relevant returned onsets to the total number of relevant onsets

$$
R=\frac{T P}{T P+F N}
$$

- F-measure (F): Recall and precision are traded at the expense of one another. If either recall or precision are very low, $\mathrm{F}$ will be as well.

$$
F=\frac{2 P R}{P+R}
$$

Results of our experiments for the onset detection technique are tabulated in Tables 2-10. Each table records the performance measures of onset detection techniques on a music recording from a single instrument mentioned at the top of the table along with the total number of notes present. 
Table 2: Performance of various onset detection methods compared to the proposed method for music file containing simple piano

\begin{tabular}{|l|c|c|c|c|c|c|c|}
\hline \multicolumn{4}{c}{ Instruments: Piano } & \multicolumn{4}{c}{ Total number of Notes: 864} \\
\hline \hline Method & TP & FP & FN & Acc & P & R & F \\
\hline IS & $60.5 \%$ & $64.2 \%$ & 341 & $29.0 \%$ & 0.358 & 0.605 & 0.450 \\
\hline WIS & $59.6 \%$ & $70.7 \%$ & 349 & $24.5 \%$ & 0.293 & 0.596 & 0.393 \\
\hline HFC & $9.5 \%$ & $67.6 \%$ & 782 & $7.9 \%$ & 0.324 & 0.095 & 0.147 \\
\hline SD & $50.8 \%$ & $63.1 \%$ & 425 & $27.2 \%$ & 0.369 & 0.508 & 0.427 \\
\hline SF & $48.1 \%$ & $59.0 \%$ & 448 & $28.5 \%$ & 0.410 & 0.481 & 0.443 \\
\hline CD & $41.3 \%$ & $59.9 \%$ & 507 & $25.6 \%$ & 0.401 & 0.413 & 0.407 \\
\hline RCD & $48.6 \%$ & $60.7 \%$ & 444 & $27.8 \%$ & 0.393 & 0.486 & 0.435 \\
\hline
\end{tabular}


Table 3: Performance of various onset detection methods compared to the proposed method for music file containing complex piano

\begin{tabular}{|l|c|c|c|c|c|c|c|}
\hline \multicolumn{4}{c}{ Instruments: Piano } & \multicolumn{5}{c}{ Total number of Notes: 571} \\
\hline Method & TP & FP & FN & Acc & P & R & F \\
\hline IS & $26.4 \%$ & $83.4 \%$ & 420 & $11.3 \%$ & 0.166 & 0.264 & 0.204 \\
\hline WIS & $48.2 \%$ & $83.5 \%$ & 296 & $14.0 \%$ & 0.165 & 0.482 & 0.246 \\
\hline HFC & $2.1 \%$ & $94.5 \%$ & 559 & $1.5 \%$ & 0.055 & 0.021 & 0.030 \\
\hline SD & $17.3 \%$ & $86.5 \%$ & 472 & $8.2 \%$ & 0.135 & 0.173 & 0.152 \\
\hline SF & $11.2 \%$ & $88.8 \%$ & 507 & $5.9 \%$ & 0.112 & 0.112 & 0.112 \\
\hline CD & $10.7 \%$ & $89.1 \%$ & 510 & $5.7 \%$ & 0.109 & 0.107 & 0.108 \\
\hline RCD & $14.0 \%$ & $87.7 \%$ & 491 & $7.0 \%$ & 0.123 & 0.140 & 0.131 \\
\hline
\end{tabular}


Table 4: Performance of various onset detection methods compared to the proposed method for music file containing simple violin

\begin{tabular}{|l|c|c|c|c|c|c|c|}
\hline \multicolumn{4}{l}{ Instruments: Violin } & \multicolumn{4}{c}{ Total number of Notes: 421} \\
\hline Method & TP & FP & FN & Acc & P & R & F \\
\hline IS & $50.4 \%$ & $88.3 \%$ & 209 & $10.5 \%$ & 0.117 & 0.504 & 0.190 \\
\hline WIS & $48.0 \%$ & $88.8 \%$ & 219 & $10.0 \%$ & 0.112 & 0.480 & 0.181 \\
\hline HFC & $61.0 \%$ & $87.7 \%$ & 164 & $11.4 \%$ & 0.123 & 0.610 & 0.205 \\
\hline SD & $41.8 \%$ & $90.7 \%$ & 245 & $8.2 \%$ & 0.013 & 0.418 & 0.152 \\
\hline SF & $42.3 \%$ & $90.4 \%$ & 243 & $8.5 \%$ & 0.096 & 0.423 & 0.156 \\
\hline CD & $70.8 \%$ & $86.0 \%$ & 123 & $13.2 \%$ & 0.14 & 0.708 & 0.233 \\
\hline RCD & $51.8 \%$ & $89.5 \%$ & 203 & $9.6 \%$ & 0.105 & 0.518 & 0.175 \\
\hline
\end{tabular}


Table 5: Performance of various onset detection methods compared to the proposed method for music file containing complex violin

\begin{tabular}{|l|c|c|c|c|c|c|c|}
\hline \multicolumn{9}{l}{ Instruments: Violin } \\
\hline Method & TP & FP & FN & Acc & P & R & F \\
\hline IS & $26.6 \%$ & $79.6 \%$ & 1178 & $13.0 \%$ & 0.204 & 0.266 & 0.231 \\
\hline WIS & $24.3 \%$ & $80.9 \%$ & 1214 & $12.0 \%$ & 0.191 & 0.243 & 0.214 \\
\hline HFC & $57.3 \%$ & $47.9 \%$ & 685 & $37.5 \%$ & 0.521 & 0.573 & 0.546 \\
\hline SD & $47.7 \%$ & $67.6 \%$ & 839 & $23.9 \%$ & 0.324 & 0.477 & 0.386 \\
\hline SF & $24.2 \%$ & $81.5 \%$ & 1216 & $11.7 \%$ & 0.185 & 0.242 & 0.210 \\
\hline CD & $65.5 \%$ & $37.3 \%$ & 554 & $34.8 \%$ & 0.427 & 0.655 & 0.517 \\
\hline RCD & $41.0 \%$ & $72.2 \%$ & 947 & $19.9 \%$ & 0.278 & 0.410 & 0.331 \\
\hline
\end{tabular}


Table 6: Performance of various onset detection methods compared to the proposed method for music file containing simple flute

\begin{tabular}{|l|c|c|c|c|c|c|c|}
\hline \multicolumn{4}{c}{ Instruments: Flute } & \multicolumn{4}{c}{ Total Number of notes: 373} \\
\hline Method & TP & FP & FN & Acc & P & R & F \\
\hline IS & $26.3 \%$ & $91.2 \%$ & 275 & $7.00 \%$ & 0.088 & 0.263 & 0.132 \\
\hline WIS & $41.3 \%$ & $87.5 \%$ & 219 & $10.6 \%$ & 0.125 & 0.413 & 0.192 \\
\hline HFC & $37.3 \%$ & $85.3 \%$ & 234 & $11.8 \%$ & 0.147 & 0.373 & 0.211 \\
\hline SD & $33.8 \%$ & $88.0 \%$ & 247 & $9.70 \%$ & 0.120 & 0.338 & 0.177 \\
\hline SF & $44.0 \%$ & $85.1 \%$ & 209 & $12.5 \%$ & 0.149 & 0.440 & 0.422 \\
\hline CD & $75.9 \%$ & $82.1 \%$ & 90 & $16.9 \%$ & 0.179 & 0.759 & 0.290 \\
\hline RCD & $52.0 \%$ & $85.5 \%$ & 179 & $13.1 \%$ & 0.150 & 0.520 & 0.233 \\
\hline
\end{tabular}


Table 7: Performance of various onset detection methods compared to the proposed method for music file containing complex flute

\begin{tabular}{|l|c|c|c|c|c|c|c|}
\hline \multicolumn{4}{l}{ Instruments:Flute } & \multicolumn{4}{c}{ Total Number of notes : 274} \\
\hline Method & TP & FP & FN & Acc & P & R & F \\
\hline IS & $37.4 \%$ & $80.7 \%$ & 179 & $14.2 \%$ & 0.193 & 0.347 & 0.248 \\
\hline WIS & $27.0 \%$ & $85.0 \%$ & 200 & $10.7 \%$ & 0.150 & 0.270 & 0.193 \\
\hline HFC & $74.8 \%$ & $68.2 \%$ & 69 & $28.7 \%$ & 0.318 & 0.748 & 0.446 \\
\hline SD & $11.3 \%$ & $92.7 \%$ & 243 & $4.6 \%$ & 0.073 & 0.113 & 0.089 \\
\hline SF & $14.6 \%$ & $90.1 \%$ & 234 & $6.3 \%$ & 0.099 & 0.146 & 0.118 \\
\hline CD & $81.4 \%$ & $67.6 \%$ & 51 & $30.2 \%$ & 0.324 & 0.814 & 0.464 \\
\hline RCD & $35.4 \%$ & $80.6 \%$ & 177 & $14.3 \%$ & 0.194 & 0.354 & 0.251 \\
\hline
\end{tabular}


Table 8: Performance of various onset detection methods compared to the proposed method for music file containing simple guitar

\begin{tabular}{|l|c|c|r|r|r|r|c|}
\hline \multicolumn{4}{l}{ Instruments:Guitar } & \multicolumn{4}{c}{ Total Number of notes: 172} \\
\hline Method & TP & FP & FN & Acc & P & R & F \\
\hline IS & $99.4 \%$ & $66.2 \%$ & 1 & $37.7 \%$ & 0.378 & 0.994 & 0.548 \\
\hline WIS & $75.6 \%$ & $78.7 \%$ & 42 & $19.9 \%$ & 0.213 & 0.756 & 0.332 \\
\hline HFC & $99.4 \%$ & $0.0 \%$ & 1 & $99.4 \%$ & 1.0 & 0.994 & 0.997 \\
\hline SD & $99.4 \%$ & $62.3 \%$ & 1 & $37.7 \%$ & 0.377 & 0.994 & 0.547 \\
\hline SF & $99.4 \%$ & $55.8 \%$ & 1 & $44.1 \%$ & 0.442 & 0.994 & 0.612 \\
\hline CD & $99.4 \%$ & $53.2 \%$ & 1 & $46.7 \%$ & 0.468 & 0.994 & 0.637 \\
\hline RCD & $99.4 \%$ & $58.5 \%$ & 1 & $41.1 \%$ & 0.412 & 0.994 & 0.583 \\
\hline
\end{tabular}


Table 9: Performance of various onset detection methods compared to the proposed method for music file containing complex guitar

\begin{tabular}{|l|c|c|r|r|r|r|c|}
\hline \multicolumn{4}{l}{ Instruments:Guitar } & \multicolumn{9}{c}{ Total Number of notes: 434} \\
\hline Method & TP & FP & FN & Acc & P & R & F \\
\hline IS & $79.5 \%$ & $68.7 \%$ & 89 & $29.0 \%$ & 0.313 & 0.795 & 0.449 \\
\hline WIS & $62.2 \%$ & $80.8 \%$ & 164 & $17.2 \%$ & 0.192 & 0.622 & 0.293 \\
\hline HFC & $80.2 \%$ & $18.9 \%$ & 86 & $67.6 \%$ & 0.811 & 0.802 & 0.806 \\
\hline SD & $84.1 \%$ & $60.2 \%$ & 69 & $37.0 \%$ & 0.398 & 0.841 & 0.540 \\
\hline SF & $82.9 \%$ & $54.6 \%$ & 74 & $41.5 \%$ & 0.454 & 0.829 & 0.587 \\
\hline CD & $82.9 \%$ & $55.9 \%$ & 74 & $40.4 \%$ & 0.441 & 0.829 & 0.576 \\
\hline RCD & $81.8 \%$ & $59.2 \%$ & 79 & $37.4 \%$ & 0.408 & 0.818 & 0.544 \\
\hline
\end{tabular}


Table 10: Performance of various onset detection methods compared to the proposed method for music file containing mixed signal

\begin{tabular}{|l|c|c|c|c|c|c|c|}
\hline \multicolumn{4}{c}{ Instruments:Mixed } & \multicolumn{4}{c}{ Total Number of notes: 1101} \\
\hline Method & TP & FP & FN & Acc & P & R & F \\
\hline IS & $80.8 \%$ & $65.0 \%$ & 211 & $32.2 \%$ & 0.350 & 0.808 & 0.488 \\
\hline WIS & $76.2 \%$ & $67.4 \%$ & 262 & $29.6 \%$ & 0.326 & 0.762 & 0.457 \\
\hline HFC & $57.9 \%$ & $41.1 \%$ & 464 & $41.2 \%$ & 0.589 & 0.579 & 0.584 \\
\hline SD & $74.6 \%$ & $65.0 \%$ & 280 & $31.2 \%$ & 0.350 & 0.746 & 0.476 \\
\hline SF & $73.6 \%$ & $59.9 \%$ & 291 & $35.1 \%$ & 0.401 & 0.736 & 0.519 \\
\hline CD & $67.6 \%$ & $58.9 \%$ & 357 & $34.3 \%$ & 0.411 & 0.676 & 0.511 \\
\hline RCD & $74.4 \%$ & $61.8 \%$ & 282 & $33.8 \%$ & 0.382 & 0.744 & 0.505 \\
\hline
\end{tabular}




\subsection{Discussions}

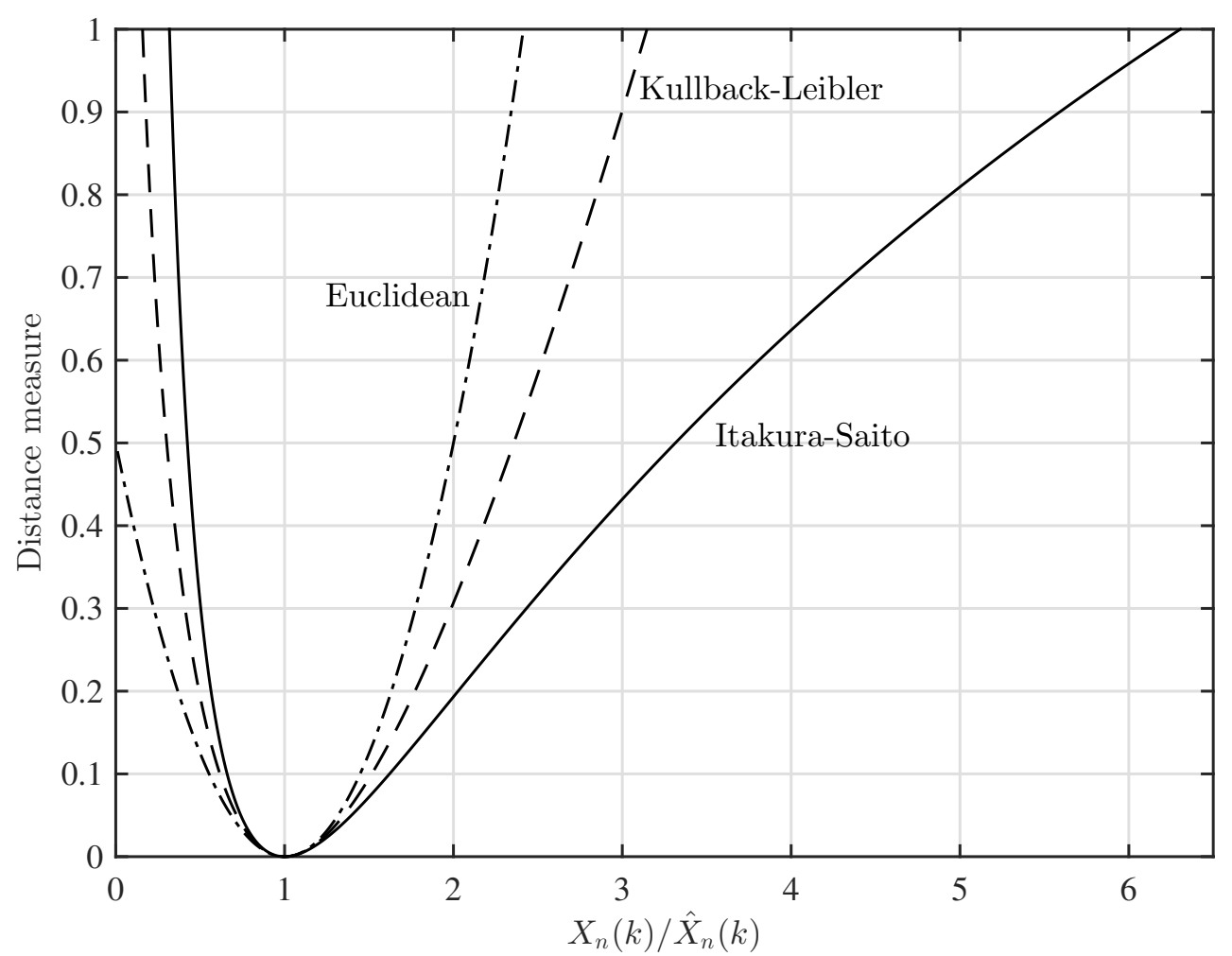

Figure 8: Performance of various distance measures

In Figure 8, some commonly used distance and divergence measures are plotted with respect to changing $X_{n}(k) / \hat{X}_{n}(k)$. These measures are given as

- Squared Euclidean (half): $\frac{1}{2}\left(X_{n}(k)-\hat{X}_{n}(k)\right)^{2}$

- Modified Kullback-Leibler: $\log _{2} \frac{X_{n}(k)}{\hat{X}_{n}(k)}$

- Itakura-Saito: $\frac{X_{n}(k)}{\hat{X}_{n}(k)}-\ln \frac{X_{n}(k)}{\hat{X}_{n}(k)}-1$

where these forms correspond to $\beta$-divergences of 2,1 , and 0 , respectively. Note that for this configuration with $X_{n}(k) / \hat{X}_{n}(k)=1$ each of these measures are 0 .

An onset normally results in a sudden increase in energy in portions of the spectrum. This increase in energy is high for strong note attacks and low for weak note 
attacks. Inspecting Fig. 8, it should be clear that the different measures will react differently to how $\mathbf{X}_{n}(k) / \hat{\mathbf{X}}_{n}(k)$ changes. The Euclidean distance and Kullback-Leibler divergence measures will tend to amplify large jumps in energy while the Itakura-Saito divergence measure will temper very large energy jumps and should allow for smaller note attacks to still display prominently. The anticipation is that the Itakura-Saito divergence measure will be better at identifying weaker note onsets without being fully overshadowed by strong note onsets. Note that the Euclidean distance grows quadratically, the Kullback-Leibler divergence largely grow linearly, and the Itakura-Saito measure initially grow quickly but then continued growth is tempered by the substitution of the logarithm. Given that acoustic perception is logarithmic, the Itakura-Saito measure should better identify note changes that are perceptible. Another feature of the Itakura-Saito divergence measure is that $D_{I S}\left(\lambda \mathbf{X}_{n} \| \lambda \hat{\mathbf{X}}_{n}\right)=D_{I S}\left(\mathbf{X}_{n} \| \hat{\mathbf{X}}_{n}\right)$, so it is relative ratios in sound feature volume that are monitored.

From Tables 2-10, we see that there is no clear winner amongst the listed techniques, i.e., we do not see one technique outperforming all the others techniques. This is to be expected, as every harmonic technique is geared to identify certain types of note profiles, like for example, HFC is good at detecting higher harmonic content note profiles and energy flux is geared to detected only changes in power spectrum density of the signal. Due to this most techniques are biased to certain types of energy profiles. This effects the performance of a technique particularly when there is huge change in the type of notes observed. We can see this in the detection function, as some note onsets are stronger that others. The Itakura-Saito divergence based onset detection works by damping the stronger notes more as compared to weaker notes and since it is looking only for positive changes in energy profile, it is well suited to detect both strong and weak note onsets. This technique overcomes the drawbacks of HFC. We choose HFC to benchmark our performance, as it has been shown to give relatively good results amongst the techniques compared. HFC weights higher harmonics more as compared to lower harmonics, this causes, weaker note onsets to be masked by stronger note onsets. The Itakura-Saito overcomes this particular limitation. To demonstrate an advantage of the proposed approach, we show a 5 second segment of a music clip (from Real Love by Clean Bandit) in Fig. 9(a) and its corresponding spectrogram in Fig. 9(b). From the time series and the spectrogram, some note onsets are easily identified. However, other notes are more difficult to visually identify in the time series and are only slightly noticeable in the spectrogram. The proposed 


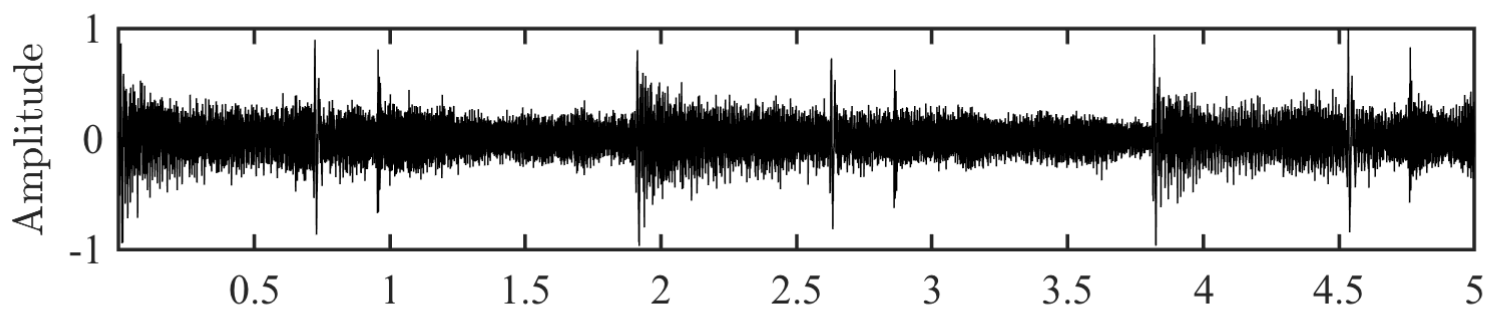

(a)

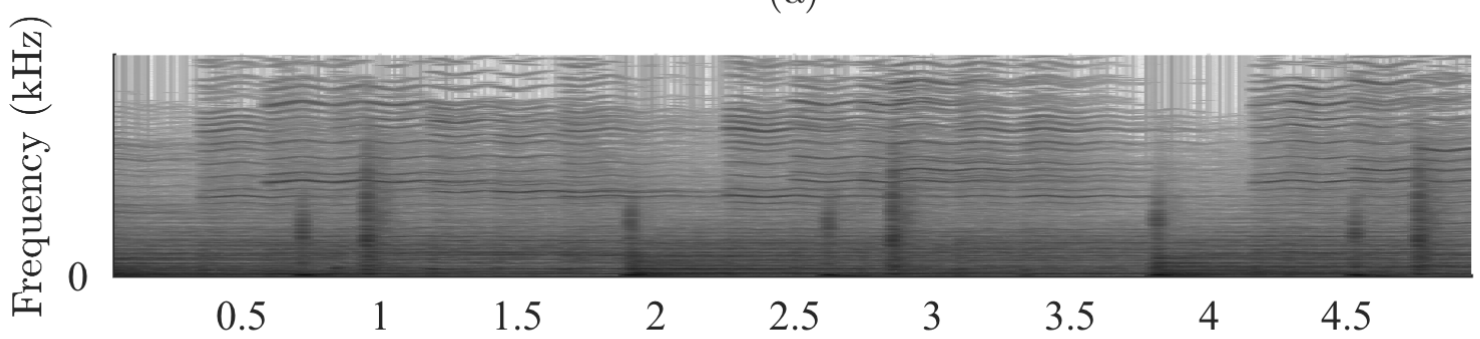

(b)

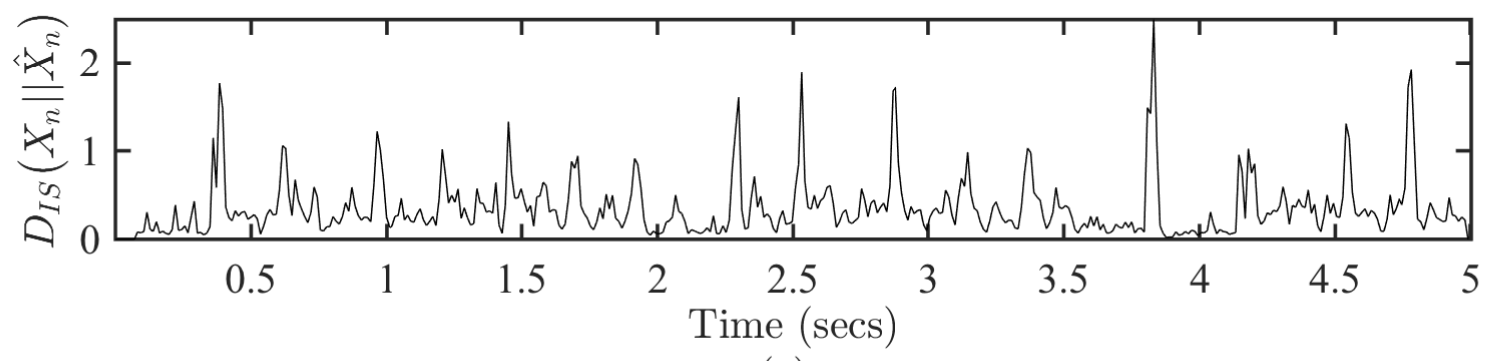

(c)

Figure 9: Performance of $D_{I S}\left(X_{n} \| \hat{X}_{n}\right)$ for on a segment of a music clip with no vocals (Real Love by Clean Bandit): (a) time series plot, (b) spectrogram, and (c) plot of $D_{I S}\left(X_{n} \| \hat{X}_{n}\right)$.

Itakura-Saito divergence measure used as a novelty function is calculated on this music segment and the results are plotted in Fig. 9(c). Appropriate peak-picking would subsequently be applied to Fig. 9(c) for the rest of the onset detection approach. We see from Fig. 9(c) that along with detecting the stronger note onset signatures in Fig. 9(b) that even weaker beats are identified such as those at approximately $0.3 \mathrm{~s}$, $0.6 \mathrm{~s}, 1.2 \mathrm{~s}, 1.45 \mathrm{~s}, 1.7 \mathrm{~s}, 2.3 \mathrm{~s}, 3.1 \mathrm{~s}, 3.4 \mathrm{~s}$, and $4.2 \mathrm{~s}$ on time axis.

The Itakura-Saito based divergence was originally developed to measure the similarity of a spectra of speech signal with its de-noised version. We see that the spectra of music signal varies greatly during an onset and/or at offset. During the transient 
part of a note, the spectra or frequency content remains fixed, with changing intensity (due to decaying amplitude in time domain signal). Hence, we propose the use of Itakura-Saito divergence to measure the similarity of the spectra about the frames of STFT of a music signal to detect note onsets. Larger distances correspond to increasing dissimilarity in notes corresponding to note onset as the half wave rectification removes distances corresponding to note offsets. To this extent, from Tables $2-10$, we can see that the Itakura-Saito based onset detection has relatively good true positive rates. We also see that some onset detection functions such as the HFC perform well for music with higher frequency content, and not so well for music with lower frequency content. This is due to the fact that HFC emphasizes higher frequencies over lower frequencies. We notice the Itakura-Saito based onset detection suffers from high false positives rates. This is due to the varying nature of amplitude during the evolution of a note. Two windows capturing the same note, will capture different parts of the note transient. As a result, both frames will contain about the same frequencies corresponding to the note, but with different intensities due to decaying nature of note. Also, some amount of spectral leakage in each frame will contribute to false positives. One approach to reduce the false positives, would be to threshold the the Itakura-Saito divergence instead of taking the half wave rectification.

The Itakura-Saito divergence measure has been shown to be highly correlated with subjective quality judgement [52]. According to a recent speech codec evaluation, if the Itakura-Saito measure between two signals is less than 0.5, the difference between their mean opinion score would be less than 1.6 [53]. Itakura-Saito measure of 0.1 or less between two speech signals, would be perceived as nearly identical by the human ear. Hence, a threshold value of 0.1 or a higher value of 0.5 , may reduce the contribution of frequency bins to false positives.

We experiment with a weighted Itakura-Saito divergence for reduction. This method is labelled as WIS in Tables 2-10.

$$
D_{W I S}(n)=\sum_{k} w_{1} \cdot \frac{\left|X_{n-1}(k)\right|}{\left|X_{n+1}(k)\right|}-w_{2} \cdot \log \frac{\left|X_{n-1}(k)\right|}{\left|X_{n+1}(k)\right|}-1
$$

Weights $w_{1}=0.04$ and $w_{2}=0.4$ are found to give best results. These values are selected empirically. From Tables $2-10$, we see that the performance of weighted 
Itakura-Saito divergence is relatively close to the onsets detected using simple ItakuraSaito divergence measure. We also see in Table 6, the wighted Itakura-Saito divergence measure performs better than the vanilla flavoured Itakura-Saito based divergence measure. 


\section{Chapter 4}

\section{Variable Window Based STFT for NMF Decomposition}

In this chapter we discuss in detail the algorithm we have developed for variable length window based short time Fourier transform (STFT) for non-negative matrix factorization (NMF).

Fig. 10 gives a brief overview of the process of obtaining the new $\mathbf{W}$ matrix. The proposed algorithm first computes the magnitude short time Fourier transform (STFT) with fixed length windows. Let us call this "fixed length window STFT". Fixed length window STFT is then fed to an onset detection algorithm to obtain a good estimate of note onsets in the given piece of recorded music. A single window is applied on the recorded time domain signal between two consecutive note onsets. In this method the variable length window STFT is computed. The absolute value of this gives us the magnitude STFT, and henceforth we will call this the "variable length window STFT" for the sake of simplicity. NMF decomposes the magnitude

STFT $\mathbf{X} \in \mathbb{R}_{\geq 0}^{M \times N}$ into two matrices $\mathbf{W} \in \mathbb{R}_{\geq 0}^{M \times K}$ and $\mathbf{H} \in \mathbb{R}_{\geq 0}^{K \times N}$ such that the product of $\mathbf{W}$ and $\mathbf{H}$ is an approximation of $\mathbf{X}$. The value of $K \leq \min (M, N)$ is selected by the user. For note extraction, the value of $K$ is chosen to represent the number of notes that might be present in the recorded piece of music. The $\mathbf{W}$ and $\mathbf{H}$ are randomly initialized before running the NMF.

If we run the NMF twice on the same input matrix with fixed $k$ and initial $\mathbf{W}$ and $\mathbf{H}$ matrices, we see different $\mathbf{W}$ and $\mathbf{H}$ matrices. On close observation, we see that every run gives us a permutation of the columns and rows of $\mathbf{W}$ and $\mathbf{H}$ matrix respectively. In order to keep the sequence of premise vectors fixed in $\mathbf{W}$ matrix, we make lower part of each column of $\mathbf{W}$ matrix as zeros. As zeros are not updated by 


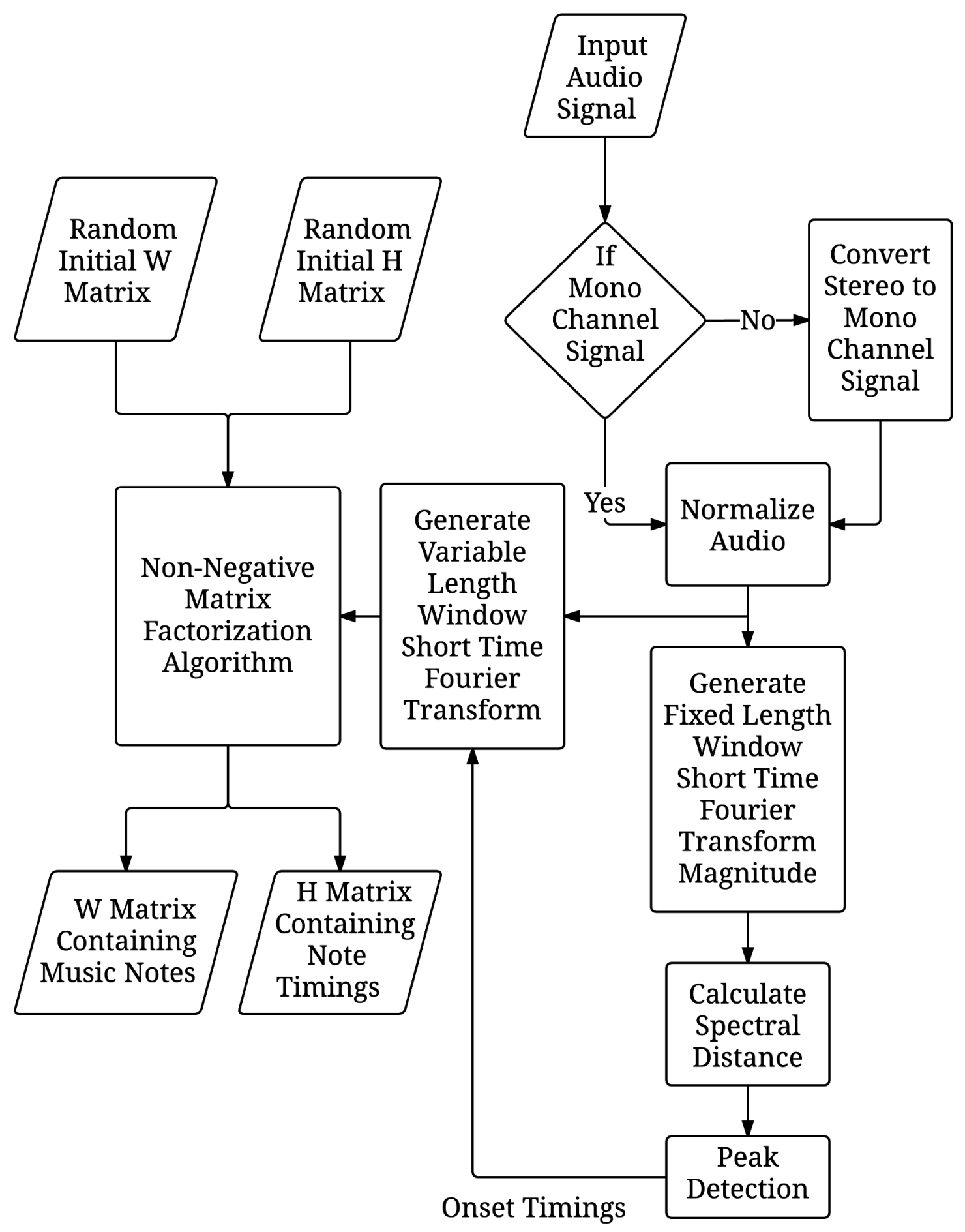

Figure 10: Variable length window based NMF scheme for music note separation 
$\mathrm{NMF}$, we are bound to get lower frequency notes in columns with lower number of zeros and higher frequency notes in columns with higher number of zeros. If we order the columns of $\mathbf{W}$ matrix with increasing number of zeros, we can get the premise vectors arranged in an increasing order based on their fundamental frequency. Here, by fundamental frequency we mean the lowest frequency that contributes to the note irrespective of its harmonic content. NMF algorithm is now run on the variable length window STFT, to get the frequency structure of the notes in $\mathbf{W}$ and their gain vectors in $\mathbf{H}$ matrix. In the next sections we describe the process in detail.

\subsection{Methodology/Algorithm}

\subsubsection{Variable Length Window Based STFT}

The onsets in a given music file are detected using any one of the onset detection algorithms available in literature. The onsets thus obtained are used to obtain a signal dependent variable length window based STFT. Input recorded music is split into frames of data such that each frame corresponds to the recorded signal between two consecutive note onsets as illustrated in Fig. 11.

Each frame is multiplied with a Hamming window and zero padded up to 131072 samples(or $2^{17}$ samples or approximately $2.97 \mathrm{~s}$ when sampled at $44.1 \mathrm{kHz}$ ). Such a large value is chosen to accommodate long duration notes such as those from violin or flute. Longer frames of data also improve the frequency resolution of longer duration signals with frequency bin width of $0.3364 \mathrm{~Hz}$ when sampled at $44.1 \mathrm{kHz}$. Discrete Fourier transform (DFT) is applied on each frame using the fast Fourier transform (FFT) algorithm. The DFT columns of time series frames are arranged in sequence to obtain variable length window based time-frequency representation using Fourier transforms. We call this variable length window based STFT, as it is STFT with variable length frame sizes.

\subsubsection{Fixed vs Variable Length Window Based STFT}

Music signals are highly dynamic in nature and can be thought of as addition of music notes in time. If we take the whole music signal, we see that it is highly non-stationary in nature, however, within the duration of a note, the frequencies contributing to that note are constant, with frequency coefficients varying in time. Due to this we can say 
that a music signals are pseudo-stationary within the notes.

As the fixed length windows do not consider the underlying nature of music, fixed length windowing is a blind way of segmenting the signal. As a result, a note might be spread over multiple windows or multiple notes might be captured in a single window. As each frame contains frequency content from multiple notes, the NMF algorithm might not converge to the basis vectors that best describe the system.

In order to get basis vectors that best describe the system, we need to capture the stationarity within a window. This can be achieved by taking windows whose length adapts to the underlying pseudo-stationarity of the signal. We choose to use note onset information to compute the variable length windows. An ideal scenario would be to consider the duration between note onsets and offsets. However, offset detection is difficult at best, due to the dynamic nature of signal (note) death. We take the distance between two consecutive onsets to give us the length of the window for that duration of the signal. The distance between two onsets effectively captures the pseudo-stationarity of the signal even with overlap of notes.

For experiments with simple notes, we developed a simple scenario with a MIDI file containing 6 notes with $30 \%$ overlap. While simple, this example should be sufficient to verify whether or not an improved factorization is obtained, and in particular whether the basis functions in $\mathbf{W}$ matrix are more accurate when using signal dependent variable length windowing versus the fixed length windowing.

The fixed window STFT is computed using a window size of 2048 samples and hop size of 1024 samples (corresponding to an overlap of 50\%). For the signal dependent variable length windows, onset detection was performed on the music recording and the results obtained are shown in Fig. 11(b). Note onsets thus obtained are used to segment the signal as shown in Fig. 11(c). This method of segmenting results in a total of 7 windows. This limited number of windows should be compared to the fixed length window approach which resulted in a total of 601 windows for this 7 second segment. Note that no overlap for the windows was used for the signal dependent variable length windowing, though it is expected that careful use of overlap would add benefits to reconstruction from the basis functions. A Hamming window was used to help reduce edge effects in the resulting STFT.

The premise vectors in $\mathbf{W}$ matrix are not arranged in a specific sequence. Thus, the arrangement of inferred notes may not be the same for each factorization. We utilize the Bhattacharyya divergence [54] to match similar notes from fixed and variable 
(a)

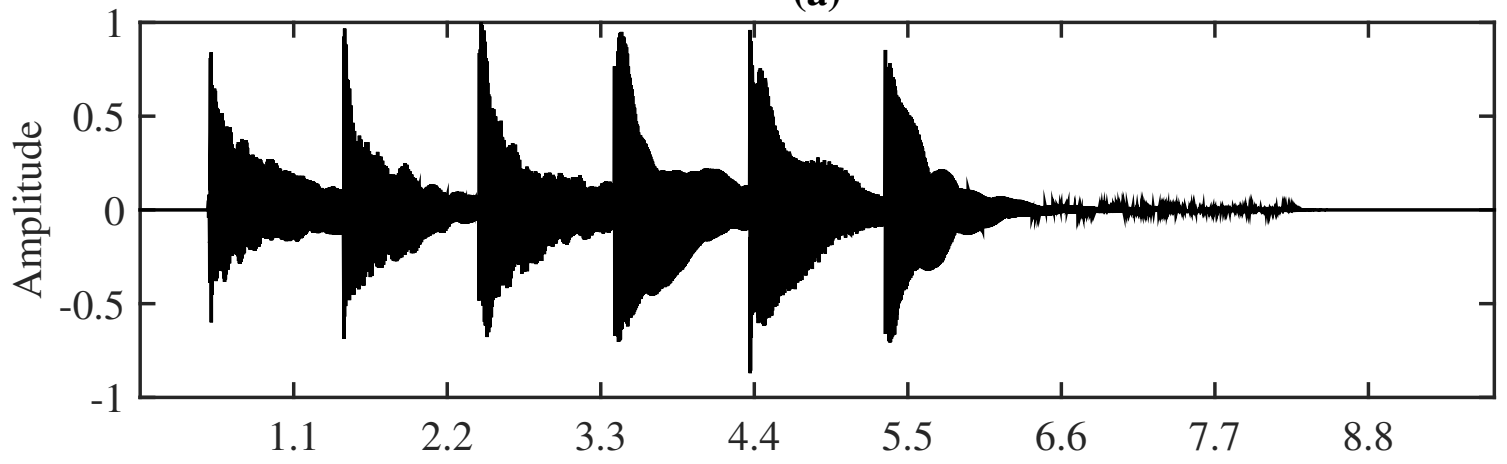

(b)

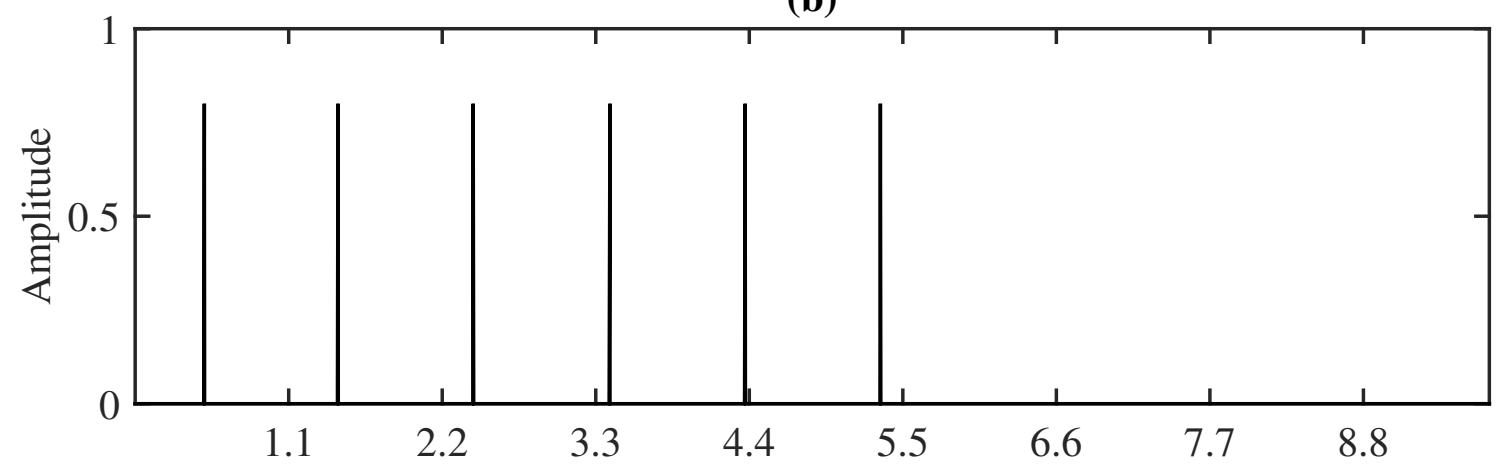

(c)

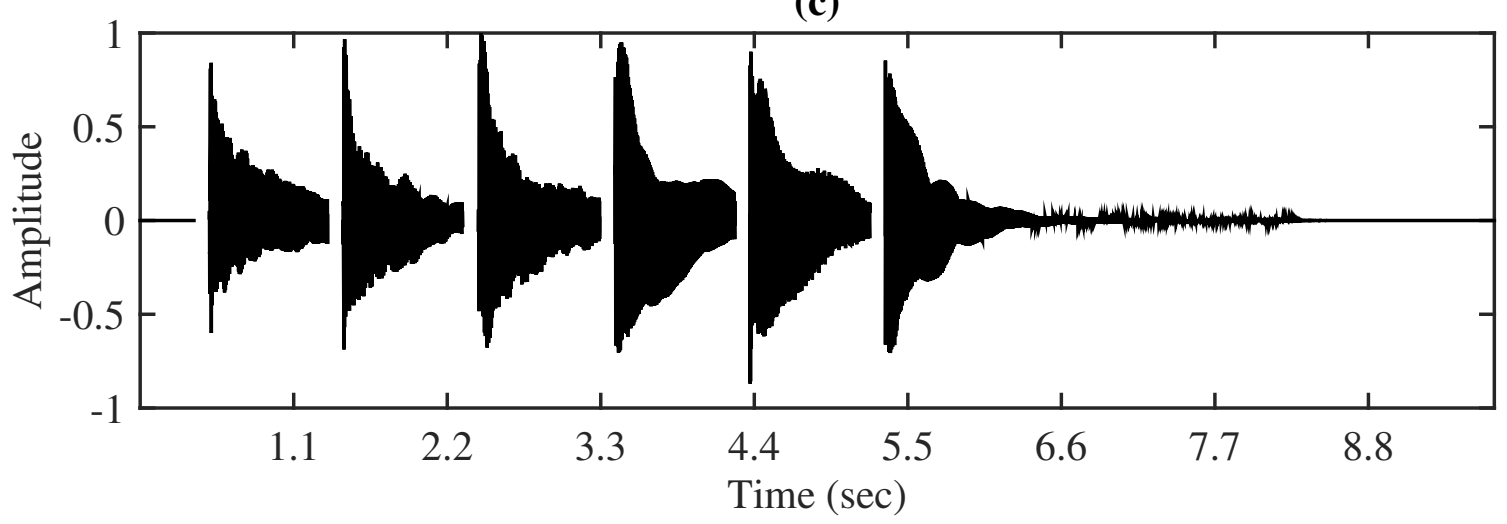

Figure 11: (a) Example time-domain plot of simple music signal. (b) Onset detection of notes for signal dependent variable length windowing. (c) Time domain signal between two consecutive onsets is segmented for signal dependent variable length window based STFT. 
length window STFT. The fixed and variable length window based STFTs for this ar-

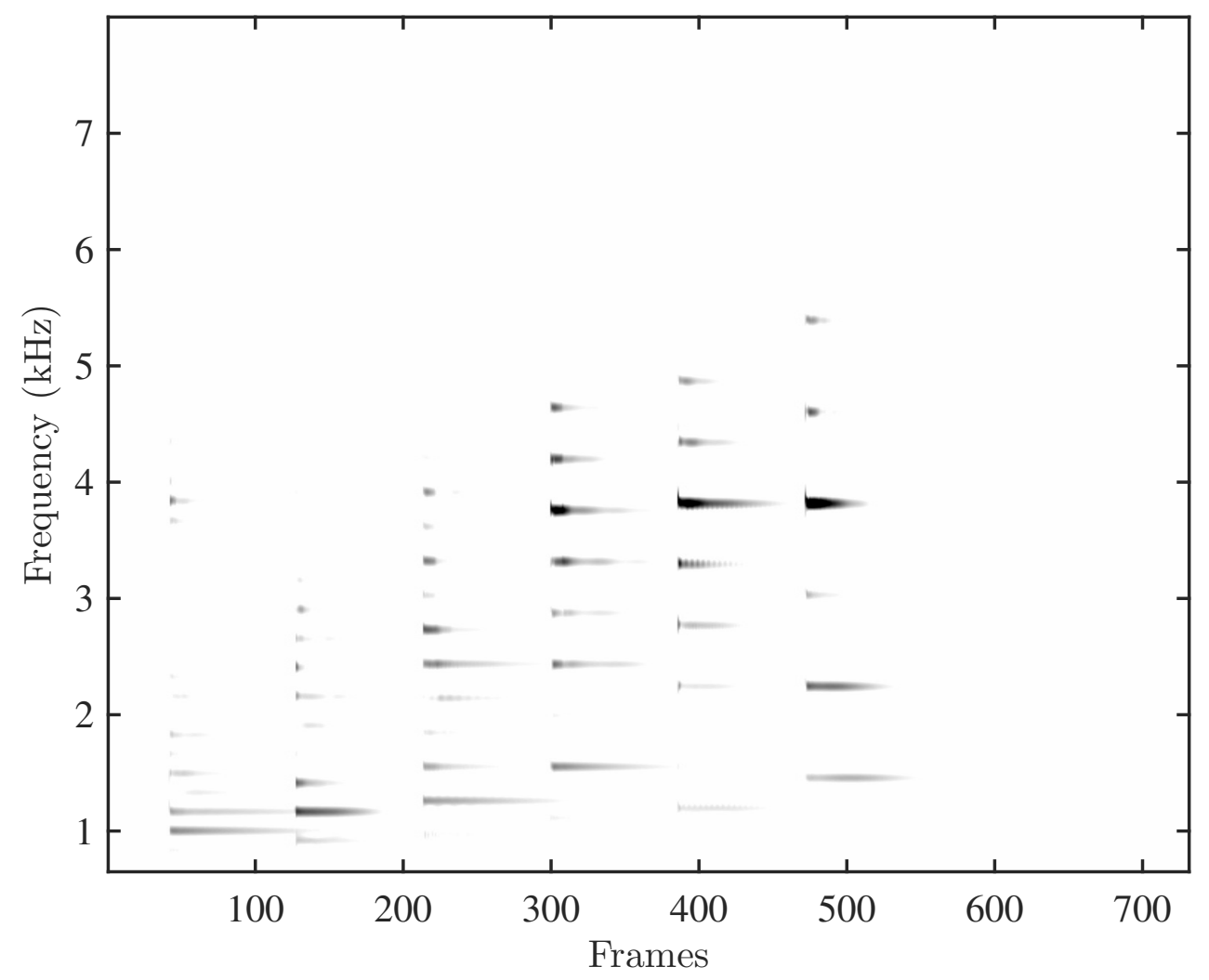

Figure 12: Fixed length window based STFT

tificial signal with 6 notes are shown in Fig. 12 and Fig. 13 respectively. From Fig. 12 we can see that similar spectral information is present in multiple frames, e.g., frames 300-350, 390-410, 480-520 and so on. This is due to multiple small frames covering relatively long duration notes. The NMF algorithm considers each frame of data to be independent of each other. As a result, higher volume of redundancy may not help with qualitative extraction of notes.

From Fig. 12, we also see that the frequency resolution is reduced when compared with variable length window based STFT in Fig. 13. This is due to smaller window size resulting in lower frequency resolution. Due the flexible length of windows, the variable length window based STFT is able to effectively capture longer notes, improving frequency resolution and reducing redundancy in STFT. 


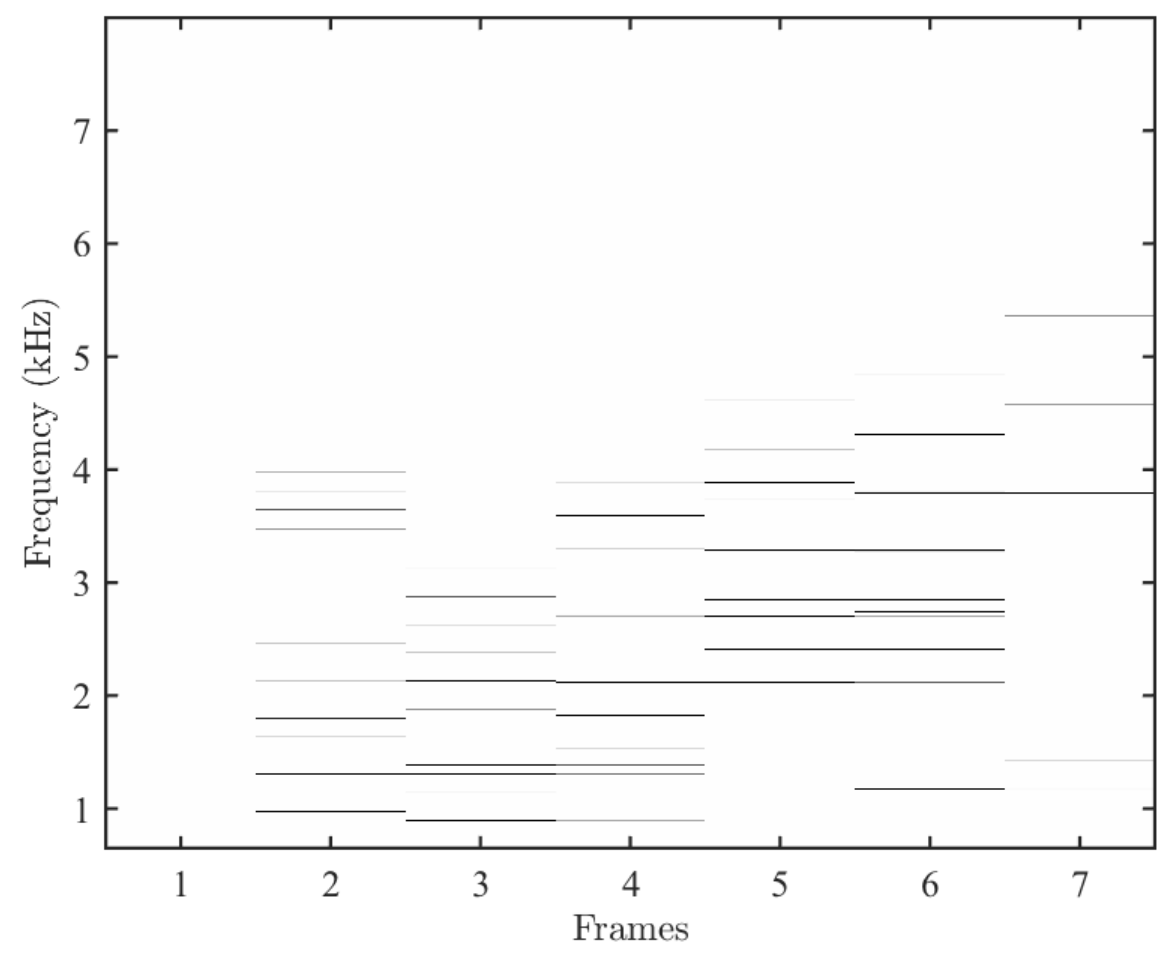

Figure 13: Variable length window based STFT

\subsection{Implementation of NMF}

The NMF algorithm takes two random matrices $\mathbf{W}$ and $\mathbf{H}$ and updates each matrix alternatively until their product is an approximation of the given time-frequency representation matrix, or the STFT. There are many update rules for $\mathbf{W}$ and $\mathbf{H}$ proposed in the literature. We will be using update rules proposed by Lee and Seung $[3,55]$.

$$
\begin{gathered}
\mathbf{W}_{i a}^{q+1}=\mathbf{W}_{i a}^{q} \frac{\left(\mathbf{X}\left(\mathbf{H}^{q}\right)^{T}\right)_{i a}}{\left(\mathbf{W}^{q} \mathbf{H}^{q}\left(\mathbf{H}^{q}\right)^{T}\right)_{i a}}, \forall i, a, \\
\mathbf{H}_{b j}^{q+1}=\mathbf{H}_{b j}^{q} \frac{\left(\left(\mathbf{W}^{q+1}\right)^{T} \mathbf{X}\right)_{b j}}{\left(\left(\mathbf{W}^{q+1}\right)^{T} \mathbf{W}^{q+1} \mathbf{H}^{q}\right)_{b j}}, \forall j, b,
\end{gathered}
$$

where $q$ is the number of iteration, $i=1, \ldots, M, j=1, \ldots, N$ and $a, b=1, \ldots, K$ The approximate $\mathbf{W}$ and $\mathbf{H}$, so obtained separate the time and frequency information from the variable length window based STFT. Each column of $\mathbf{W}$ represents the frequency spectra of derived musical notes. Whereas, each row of $\mathbf{H}$ represents the amplitude change of derived notes in time. The factorization uses an iterative method starting 
with random initial values for $\mathbf{W}$ and $\mathbf{H}$ matrices. The basis vectors in $\mathbf{W}$ matrix are ordered in decreasing length to get the vectors in increasing order of frequency spectra.

The goal of NMF is to approximate a non-negative $M \times N$ matrix $\mathbf{X} \in \mathbb{R}_{\geq 0}^{M \times N}$ as a product of two non-negative matrices $\mathbf{W} \in \mathbb{R}_{\geq 0}^{M \times K}$ and $\mathbf{H} \in \mathbb{R}_{\geq 0}^{K \times N}$, such that the error of reconstruction is minimized. Since this is an iterative process, a cost function is used to minimize the error of reconstruction. We try to minimize the root mean square of the residual $\mathrm{D}$, given as

$$
D=\|\mathbf{X}-\mathbf{W} * \mathbf{H}\|_{F} / \sqrt{N M}
$$

\subsection{Experimental Setup and Discussion on Results}

\subsubsection{Experimental Setup}

All the experiments are run on a MacBook Pro with $2.6 \mathrm{GHz}$ Intel i7 processor and $16 \mathrm{~GB} 1600 \mathrm{MHz}$ DDR3 RAM. All the test scripts are written using the DSP toolbox in MATLAB 2014b. Due to huge memory requirements by the NMF and STFT algorithms, we use the single precision available in MATLAB. This has reduced the memory requirement and has speed up the computation time over using double precision without loss of accuracy.

For experiments, we generate a signal with 6 unique notes with each note happening only once within the duration of the signal. This test signal is as shown in Fig. 11. These notes are generated using the MIDI toolbox [51] in MATLAB. Each note has significant overlap with other notes. 131072 frequency bins are computed for the STFT. Shorter windows of data are zero padded. Such a large number of frequency bins is chosen to fully capture the longer notes and to improve the frequency resolution. This however, does not reduce the spectral leakage. The STFT is computed using Hamming window. The Fourier transform is computed using the fast Fourier transform (FFT) algorithm. A ground truth table is also built using the 6 notes for comparison with the derived notes. 


\subsubsection{Analysis of W Matrix}

The resulting STFTs for both fixed and variable length windows were passed through an NMF decomposition to obtain $\mathbf{W}$ and $\mathbf{H}$. The nnmf program from MATLAB 2014b Statistical and Machine Learning Toolbox is used to factorize the STFT. The maximum number of iterations is set to 1000 and maximum residue for the cost function is chosen to be 0.0005 .

The $\mathbf{W}$ matrix derived using the fixed length window and variable length window based STFT are illustrated in Fig. 14 and Fig. 15. The basis vectors of $\mathbf{W}$ are arranged in order for comparison. We see that the basis vectors from both types of STFT share similar envelope for the frequency spectra. This leads us to believe that the use of variable length window based STFT does not affect the envelope of the frequency spectra in a significant manner. Now to consider how it affects the frequency content of the derived notes, let us have a look at the frequency spectra of a single note.

To determine the effect of variable length windows versus fixed length windows on basis functions in $\mathbf{W}$, we plot an example FFT for a note (MIDI note 79) in Fig. 16. In Fig. 16, we show the FFT for the original note in Fig. 16(a), the column from W corresponding to note 79 for the fixed length window based NMF approach in Fig. 16(b), and the column from $\mathbf{W}$ corresponding to note 79 for the variable length window based NMF approach in Fig. 16(c). For each of these, we used an extended window of length 131072 samples, but the plots only show frequency bins from $0 \mathrm{~Hz}$ to $4,200 \mathrm{~Hz}$ for display and comparison purposes.

From Fig. 16, we see some interesting results for the variable length windowing versus the fixed length windowing. First, both the variable and fixed length NMF techniques have similar primary frequency content and we see additional frequencies that are not present for the original note in Fig. 16(a), such as around $300 \mathrm{~Hz}, 500 \mathrm{~Hz}$, and $700 \mathrm{~Hz}$. This result suggests similar limitations in how the NMF decomposition actually performs on this particular input music signal.

Another observation from Fig. 16 is that there is much more spectral leakage shown in the fixed length window based NMF approach in Fig. 16(b) versus the signal dependent variable length window based NMF approach in Fig. 16(c). The

longer time windows has reduced the overall spectral leakage when using the variable 


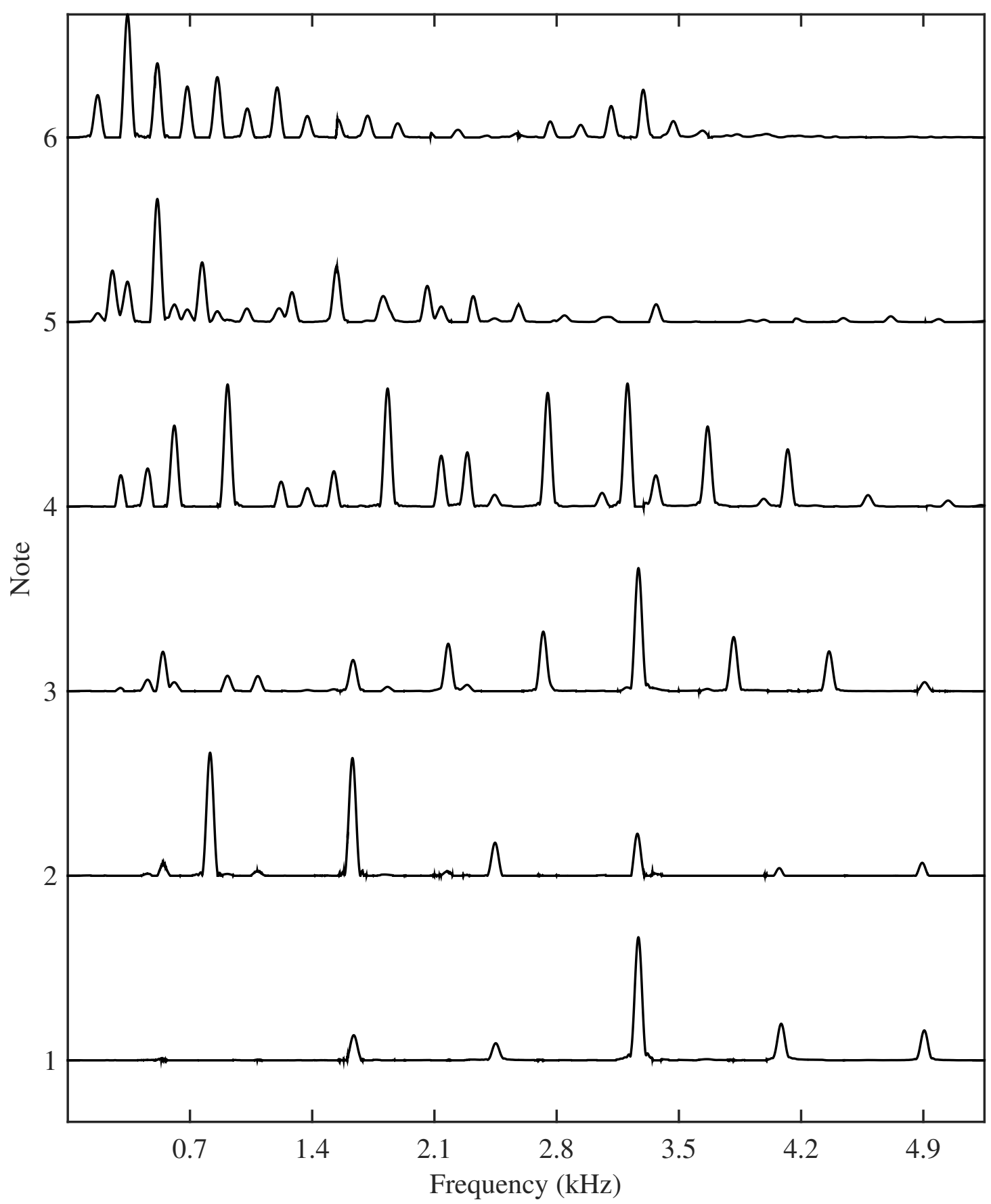

Figure 14: W matrix obtained by factorization of fixed length window based STFT 


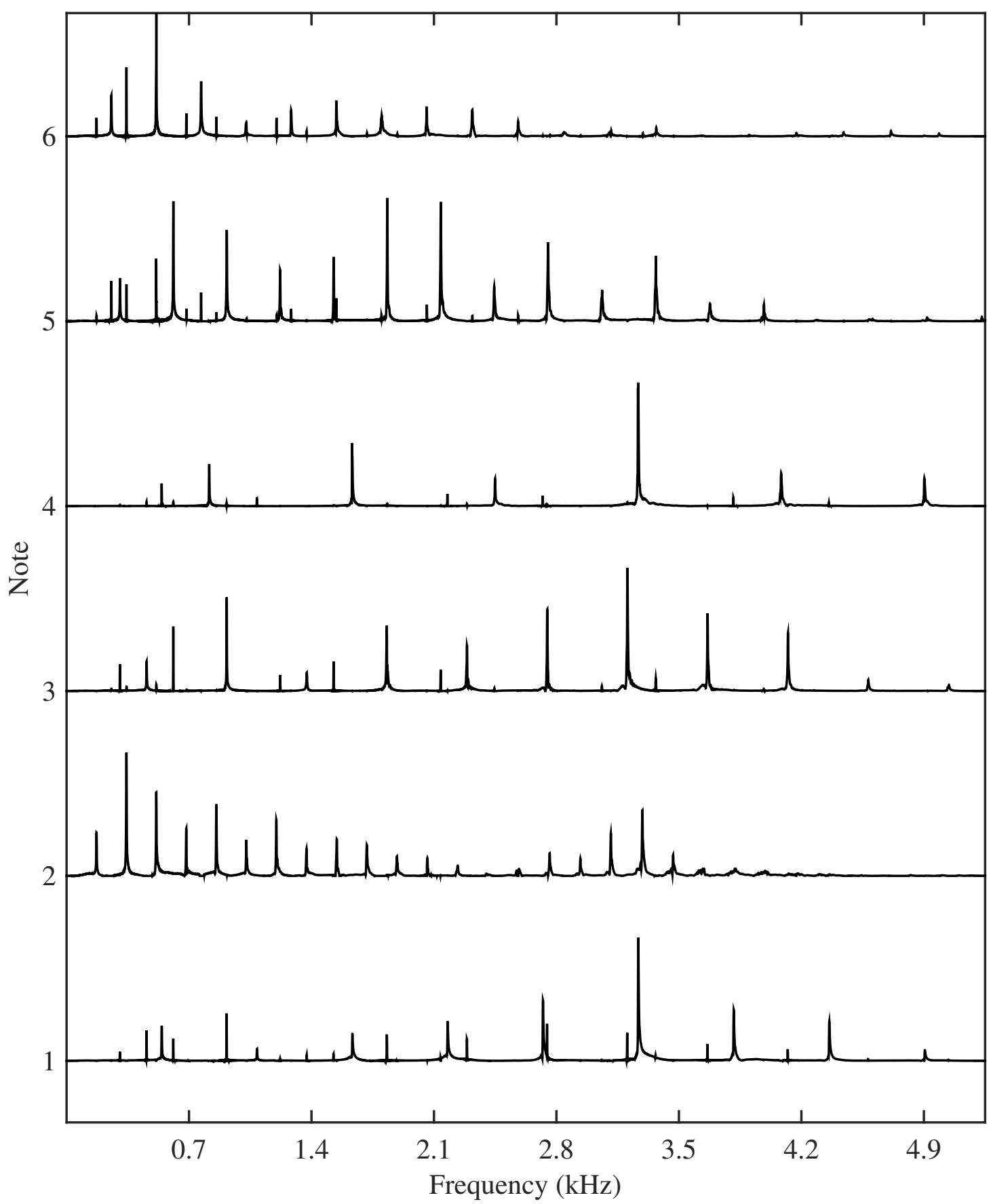

Figure 15: W matrix obtained by factorization of variable length window based STFT 

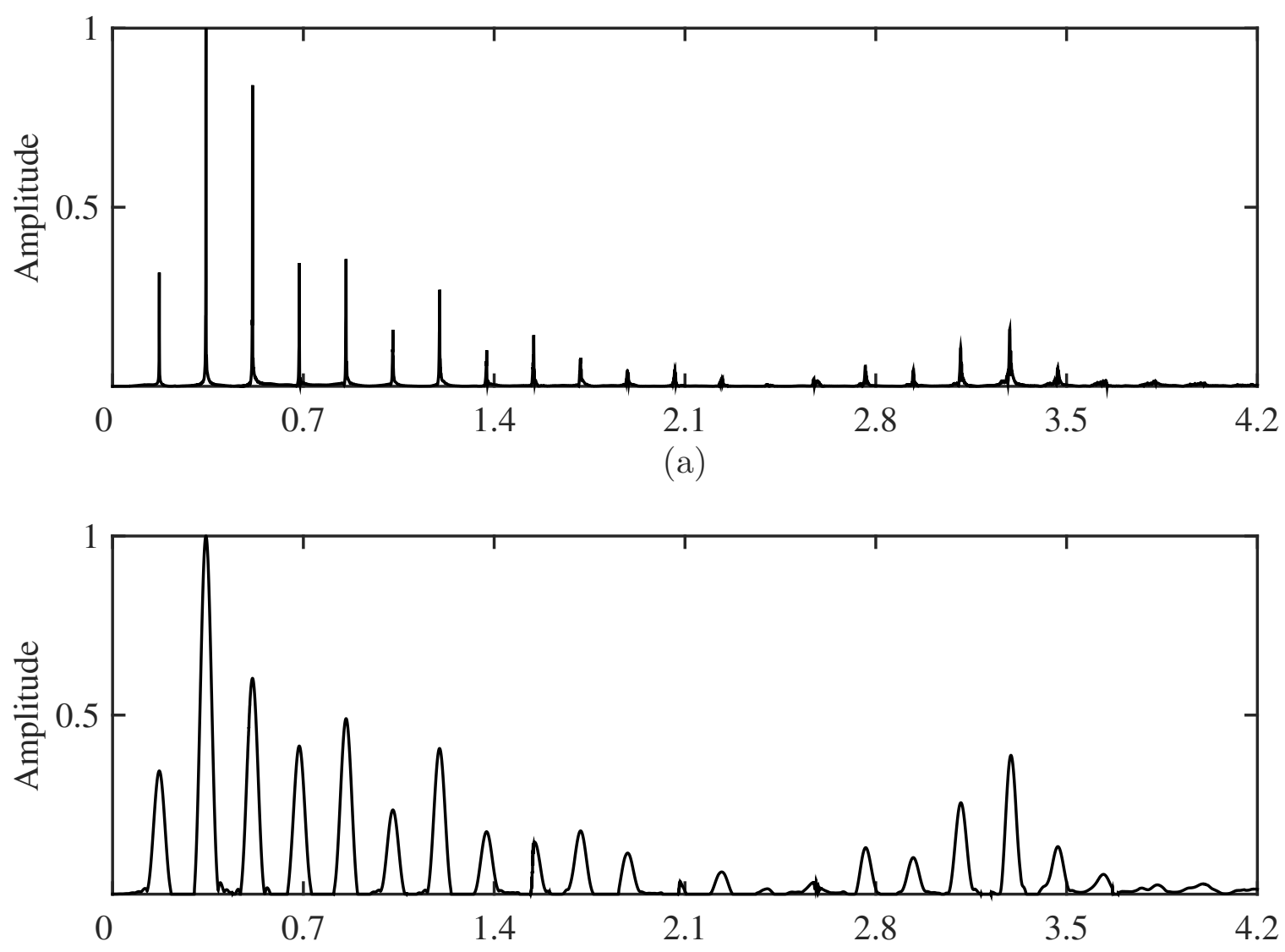

(b)

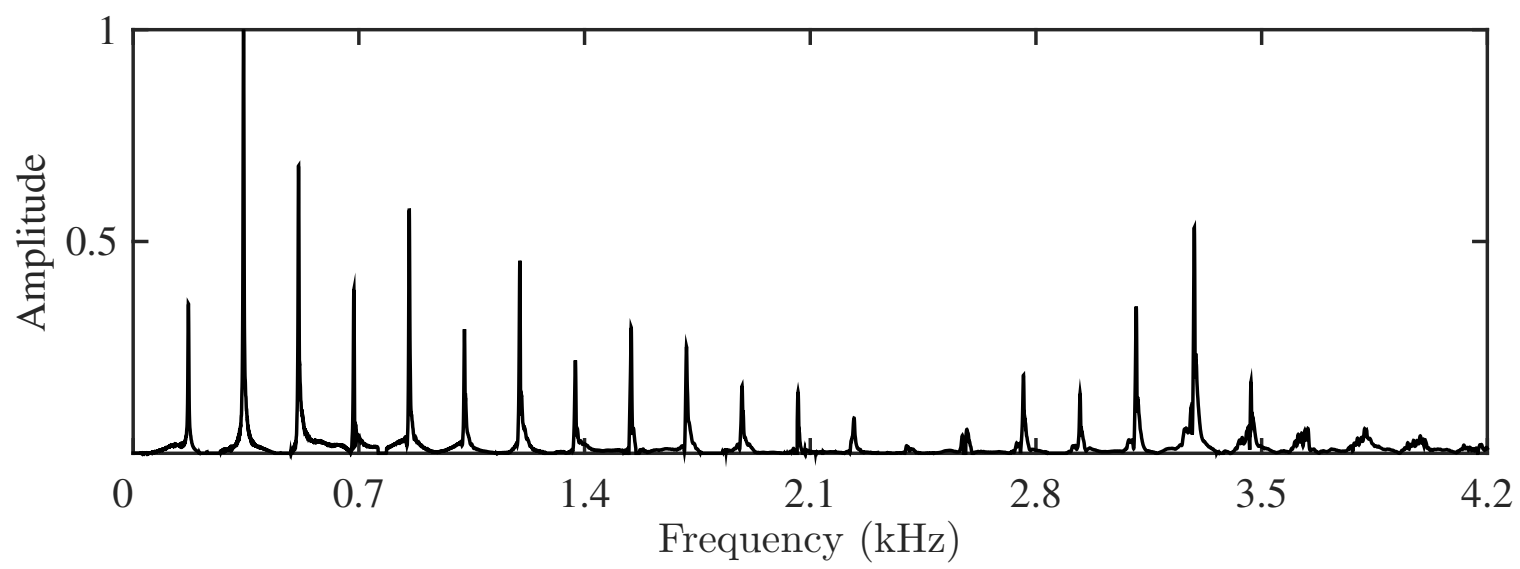

(c)

Figure 16: a) Fourier transform of MIDI note 79 corresponding to G5, b) Note no 79 derived using fixed length window STFT, and c) Note no 79 derived using variable length window STFT 
length window based NMF approach. The results in Fig. 16(c) appear to be a better match for those in Fig. 16(a).

\subsubsection{Analysis of $\mathrm{H}$ Matrix}

The non-negative factorization algorithm decomposes the given STFT X into two matrices, the $\mathbf{W}$ matrix with spectra of the derived notes and $\mathbf{H}$ matrix with the gain of these notes in time, such that the product of $\mathbf{W}$ and $\mathbf{H}$ is an approximation of $\mathbf{X}$.

$$
\mathbf{X} \approx \mathbf{W} * \mathbf{H}
$$

In the NMF with variable length window based STFT, we see that due to the variable length of windows, the amplitude envelope of the basis vector in time is lost. Instead, it is replaced by sharp peaks corresponding to the presence of these vectors in the variable window based STFT. The $\mathbf{H}$ matrix obtained by factorization of fixed length window STFT is illustrated in Fig. 17. In the fixed length window based NMF, we get an $\mathbf{H}$ matrix that somewhat reflects the amplitude change of basis vectors in $\mathbf{W}$ matrix over time. However, due to low quality of the basis vectors in $\mathbf{W}$ matrix, the $\mathbf{H}$ matrix also suffers, as the NMF tries to approximate the product of $\mathbf{W}$ and $\mathbf{H}$ to input fixed length window STFT $\mathbf{X}$. We can observe this in notes 1, 2, and 4 in Fig. 17. From the time based signal we know that no two notes happen at the same time and each note occurs only once during the entire duration of the signal. However, in the conventional $\mathbf{H}$ matrix, we see that notes 1 and 2 happen at the same time. What is really happening here is that the energy from note 1 is falsely identified with note 2 . We see that the gain vector for note 4 has two notes, implying that note 4 occurs twice in the signal, even though each note only occurs once. This is due to the poor quality of basis vectors derived by NMF from the fixed length window based STFT.

Hence, there is a scope to improve the timing information of basis vectors in $\mathbf{H}$ matrix. One way to go about it is to improve the $\mathbf{W}$ matrix, so that the $\mathbf{H}$ matrix can extract the timing information with better discrimination between the basis vectors in $\mathbf{W}$ matrix. We do this by computing the $\mathbf{H}$ matrix, while keeping the $\mathbf{W}$ matrix fixed. This fixed $\mathbf{W}$ matrix is derived by NMF using the variable length window based STFT. The new $\mathbf{H}$ matrix computed using the $\mathbf{W}$ matrix from variable length window based STFT is illustrated in Fig. 18. In this new $\mathrm{H}$ matrix we see that, 
Table 11: Bhattacharyya distance as similarity measure between derived notes in W and MIDI synthesized notes

\begin{tabular}{|c|c|c|}
\hline Note \# & Fixed STFT & Variable STFT \\
\hline 1 & 0.3976 & 0.0507 \\
\hline 2 & 0.4590 & 0.2721 \\
\hline 3 & 0.4528 & 0.3439 \\
\hline 4 & 0.4799 & 0.4177 \\
\hline 5 & 0.5053 & 0.4214 \\
\hline 6 & 0.4590 & 0.2853 \\
\hline
\end{tabular}

the previous issues are resolved. Each note only occurs once and they are all well separated in time as is the case.

\subsubsection{Similarity of Derived Notes with Ground Truth Notes}

To further test the performance of the fixed length versus variable length window approach for NMF, we computed the Bhattacharyya distance [54] between each note basis vector in $\mathbf{W}$ and the original note. The Bhattacharyya distance, given as

$$
D_{B}(p, q)=-\ln \left[\sum_{x} \sqrt{p(x) \cdot q(x)}\right]
$$

was originally developed to measure the similarity between probability distribution $p(x)$ and $q(x)$. For our purpose, we use the Bhattacharyya distance to measure the similarity between the power density spectrum estimates for each of the notes.

The results of using the Bhattacharyya distance are shown in Table 11. For the Bhattacharyya distance, if $p(x)=q(x)$ then $D_{B}(p, q)=0$. Increase in dissimilarity between $p(x)$ and $q(x)$ results in an increase for $D_{B}(p, q)$. From Table 11, we see that for all six notes that the Bhattacharyya distance $D_{B}$ is lower for signal dependent variable length window based approach for NMF. Fig. 19 gives a better visual inspection of these results. From the bar chart it is evident that some notes derived using the fixed length window based NMF that the Bhattacharyya distance appears to be 


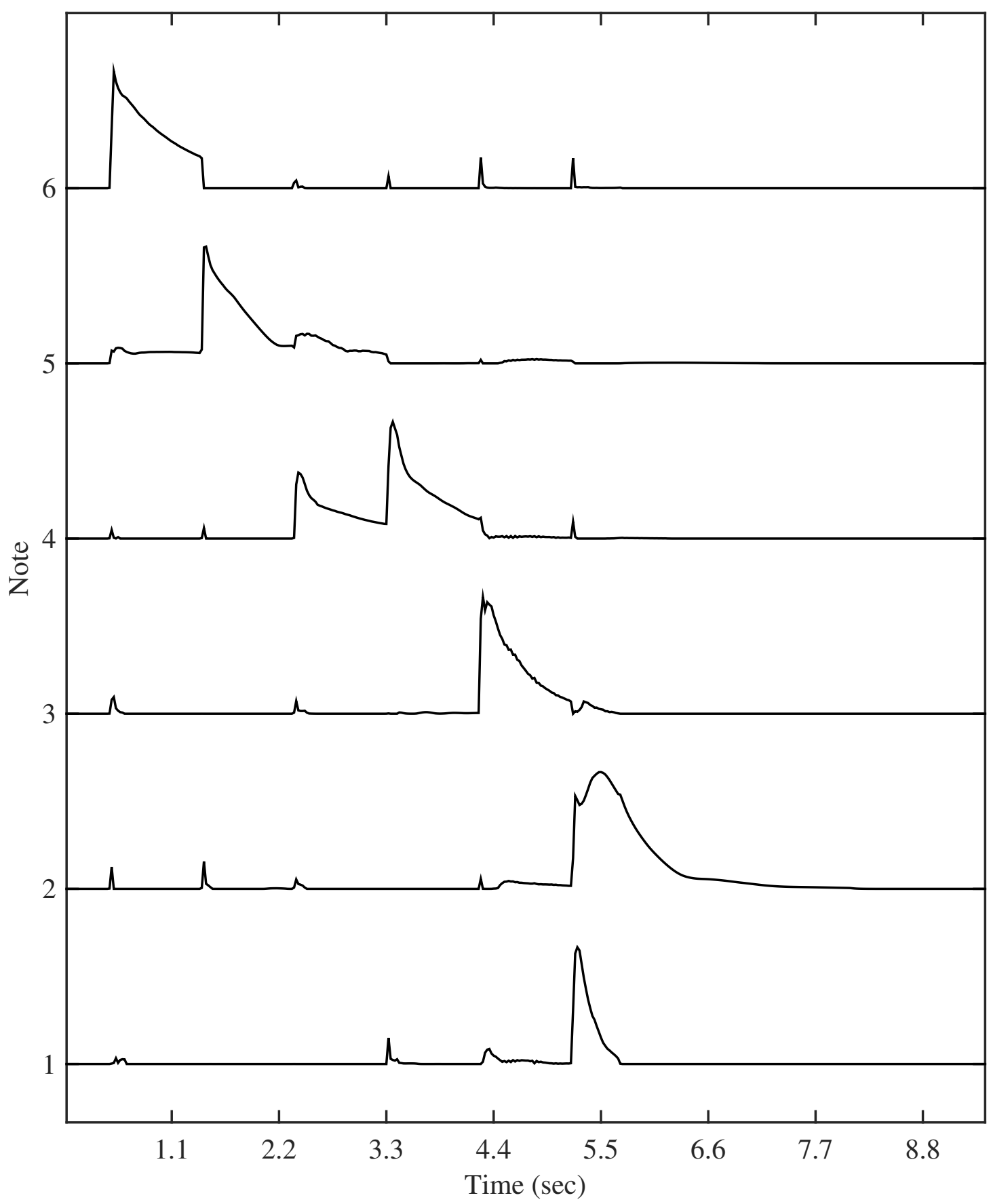

Figure 17: $\mathbf{H}$ obtained on factorization of fixed length window based STFT 


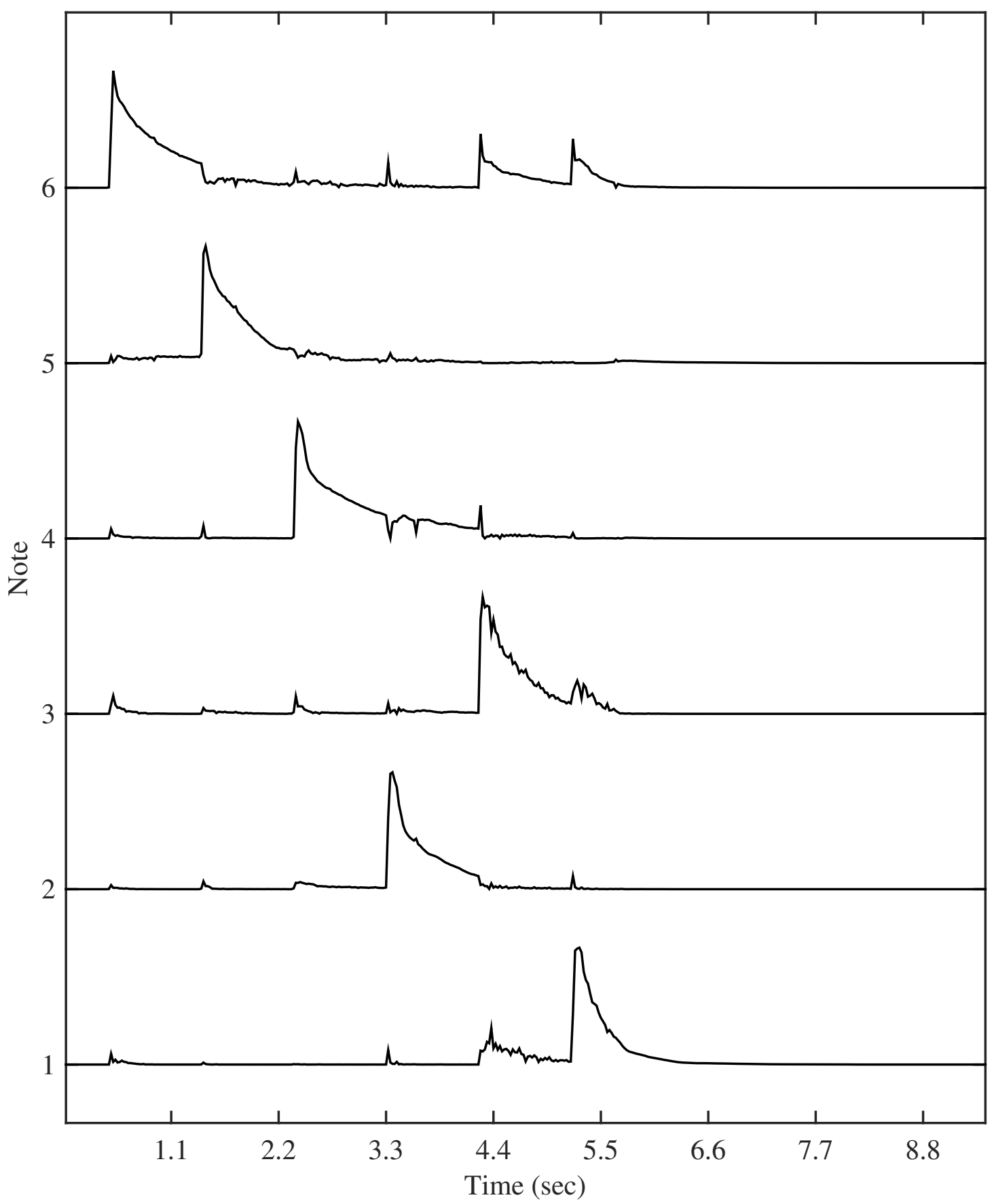

Figure 18: $\mathbf{H}$ obtained on factorization of fixed length window based STFT keeping $\mathbf{W}$ matrix fixed. This $\mathbf{W}$ matrix is available on factorization of variable length window based STFT 


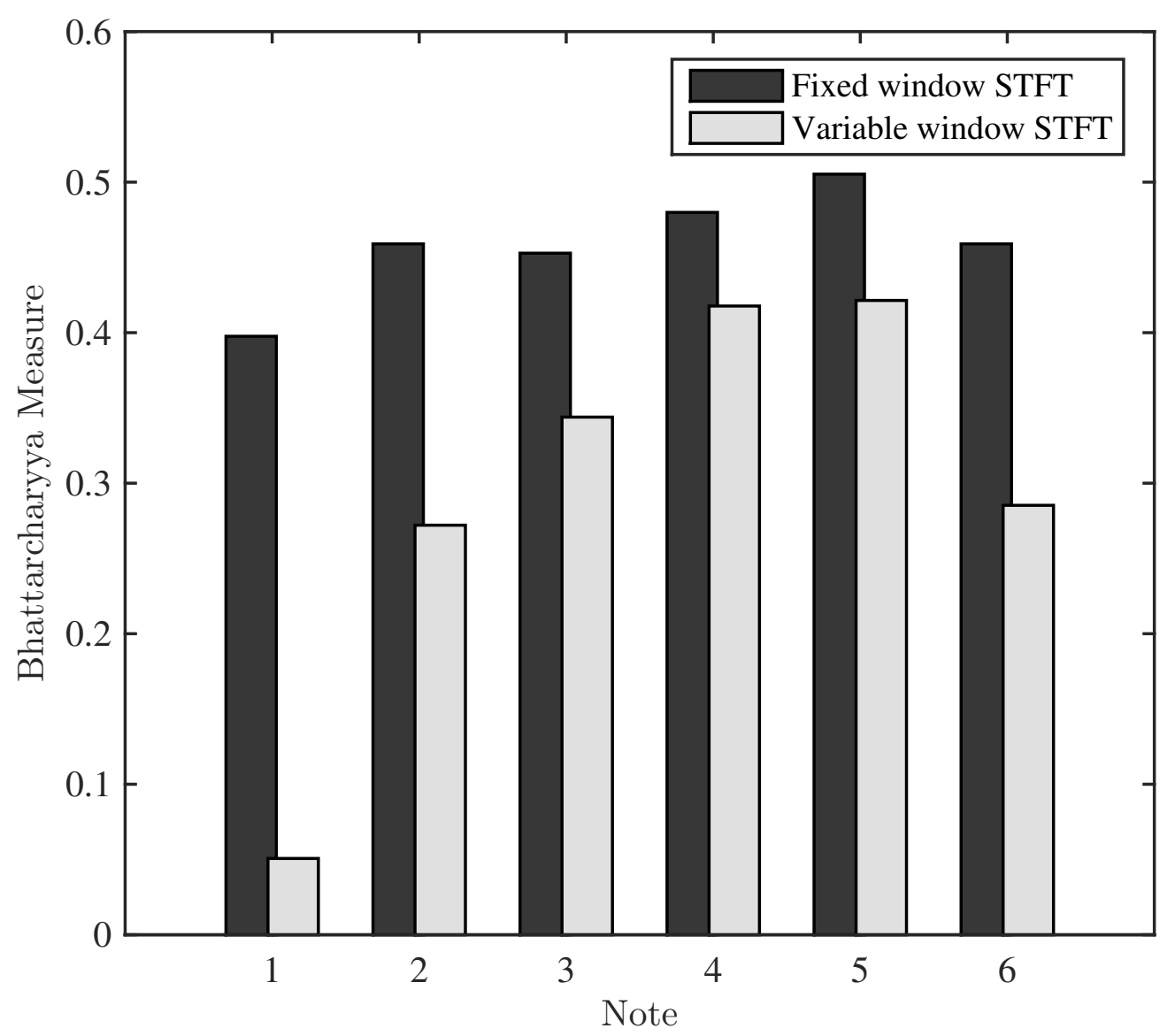

Figure 19: Bar plot of similarity measure of derived notes with ground truth notes using Bhattacharyya distance.

quite large.

\subsubsection{Analysis of Factorization of Complex Signal}

In the previous sections we discussed the results obtained using variable length window STFT and fixed length window STFT. The example signal we considered was simple and we want to measure the performance of the proposed approach on a more complicated signal, which will be a much better approximation to the real world recordings. In this section, we analyze the performance of the proposed approach on an artificially created complex signal. To create the complex signal, we are using the same 6 notes as described in the previous section and then randomize the occurrence 


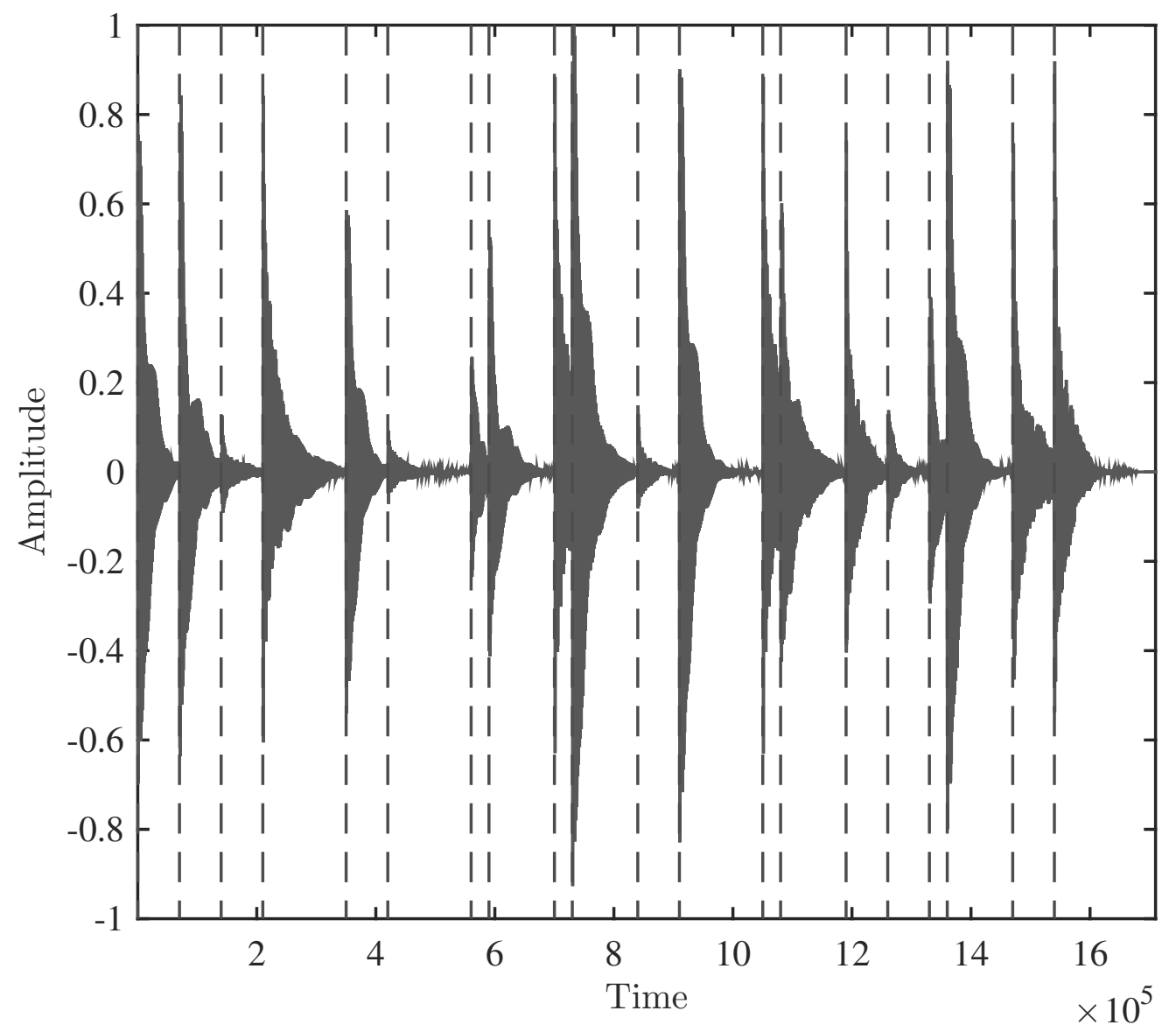

Figure 20: Artificially generated complex signal.

of these notes. This is done to have random overlap between notes. The total number of notes thus generated is limited 20 notes, for ease of analysis. These 20 notes are randomly scaled before combining them to get the complex signal. The time domain signal generated can be seen in Fig. 20 with dotted lines marking the note onsets. We can see that all the notes are randomly scaled with varying degree of overlap between them.

For the generation of fixed length window STFT, we use a window length of 2048 samples and hop size of 1024 samples resulting in an overlap of 50\%. Both fixed and variable length window STFT are computed using a Fourier transform length of 131072 samples. Such a large number of frequency bins is chosen to give good frequency resolution and to capture any long duration notes as well. We are using the nnmf program [56] to perform the non-negative matrix factorization. Number of 
notes is chosen as 6 for the NMF decomposition. Default parameters are used for the nnmf program. Next we analyze the $\mathbf{W}$ and $\mathbf{H}$ obtained on factorization of variable and fixed length window STFT.

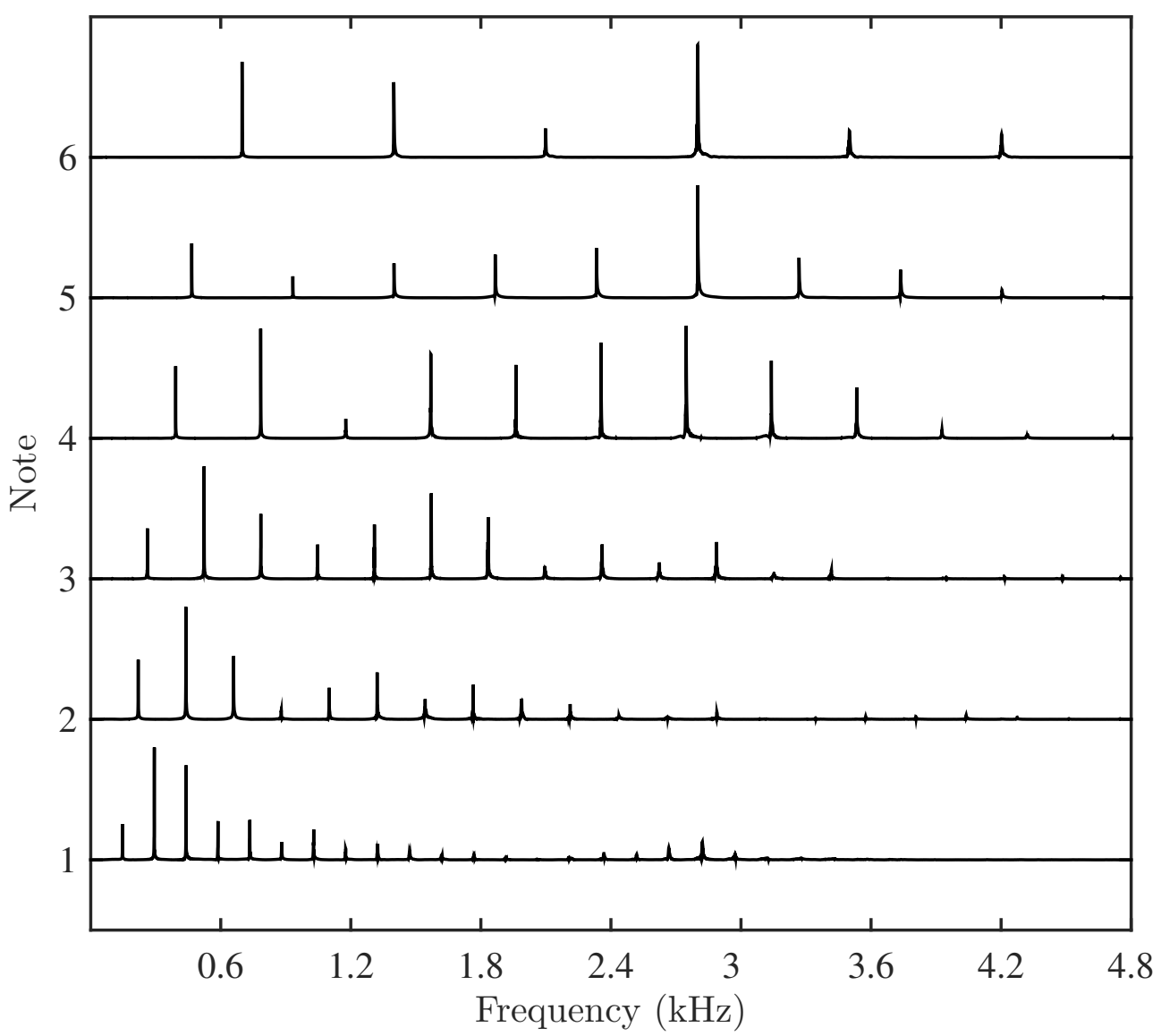

Figure 21: Frequency distribution of notes from ground truth table.

\section{W matrix computed for complex signal}

Fig. 21 plots the frequency distribution of the ground truth notes. Only frequencies bins up to $4.8 \mathrm{KHz}$ are displayed where most of the signal strength is concentrated. Fig. 22 plots the frequency distribution of the notes obtained on factorization of the fixed length window STFT. And Fig. 23 plots the the frequency distribution of notes 


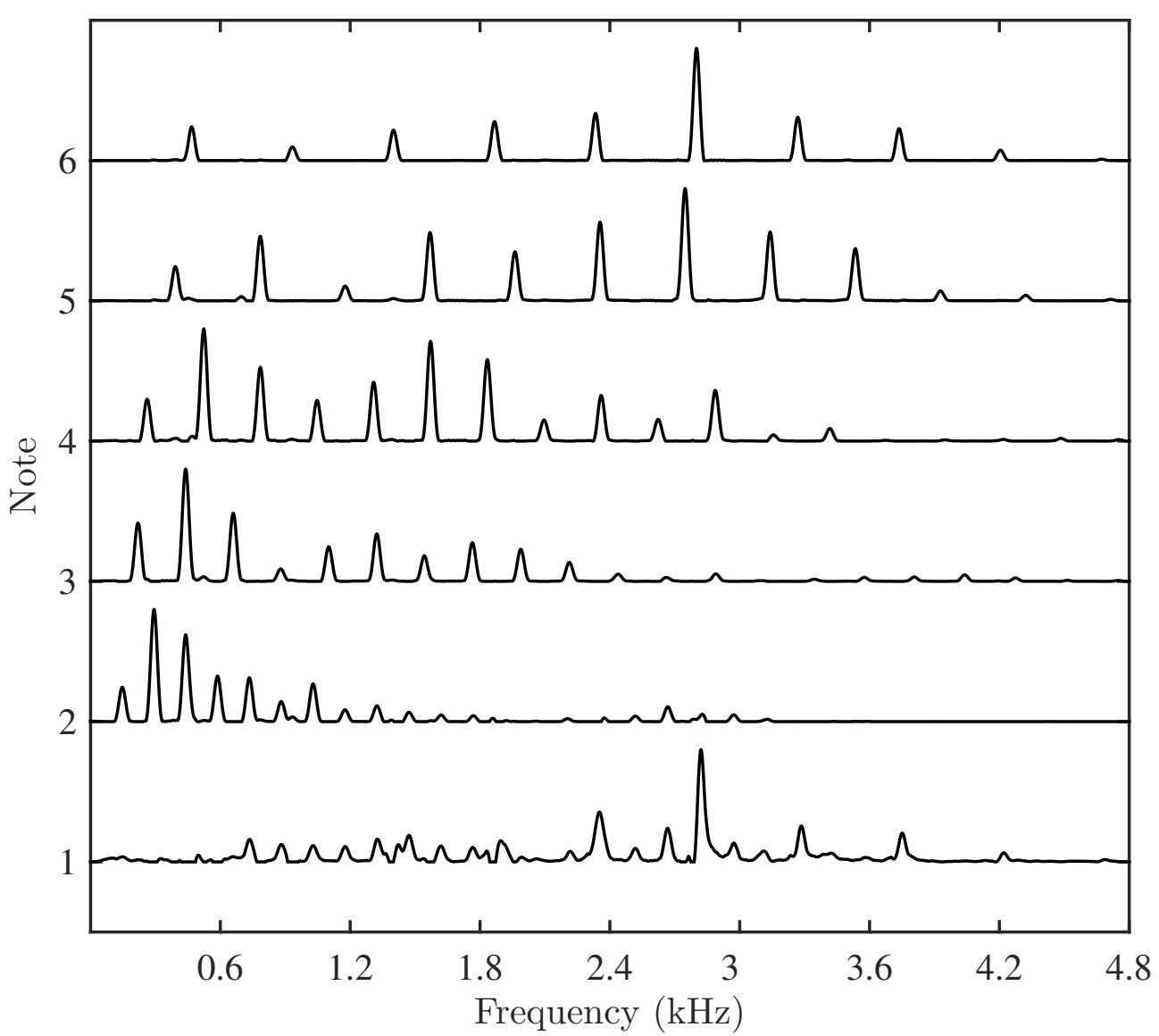

Figure 22: Frequency distribution of notes obtained from factorization of fixed length window STFT.

derived using the proposed variable length window STFT. From Fig. 23 and Fig. 22, we can spot 3 differences between the frequency distribution of notes from variable and fixed length window STFT. Firstly, the four notes $(3,4,5$ and 6$)$ look similar for both variable and fixed length window STFT, except for some spectral leakage about the primary frequencies in the notes obtained on factorization of fixed length window STFT. The second discrepancy is the frequency distribution of note 2 for both fixed and variable length window STFT. When we compare the frequency distribution of the derived notes (note number 2) from fixed and variable length window STFT with note 2 in the ground truth in Fig. 21, we see that the fixed length window STFT has damped frequency at $2.9 \mathrm{kHz}$. This is not the case when the variable length window 


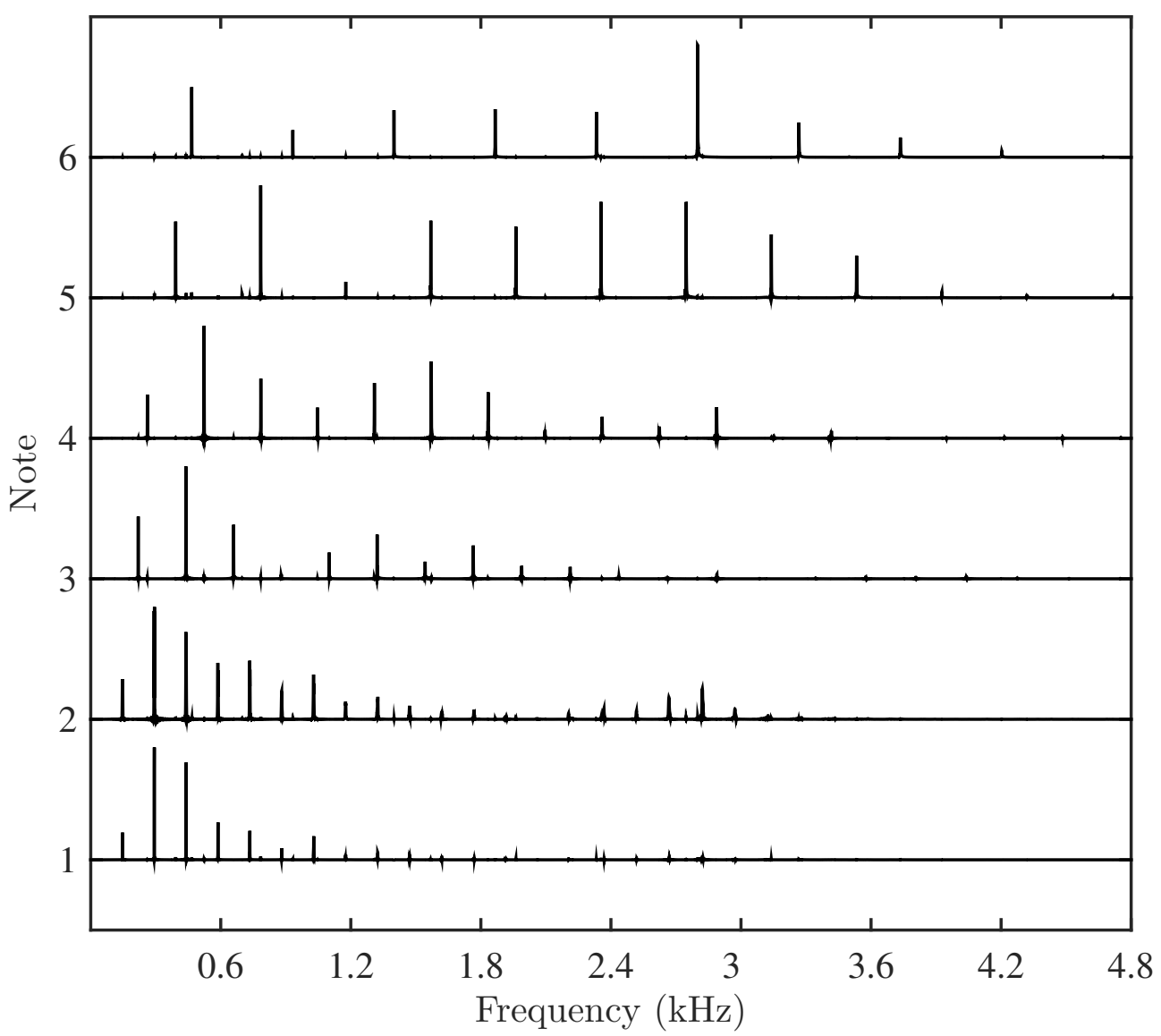

Figure 23: Frequency distribution of the notes obtained from the factorization of the variable length window STFT.

STFT is used for factorization. And lastly, we compare note 1 from the fixed and variable length window STFT to note 1 from the ground truth table in Fig. 21. We can clearly see that frequency distribution of note 1 from the fixed length window STFT is completely distorted. The variable length window STFT gives us a better approximation of the note 1 spectra from the ground truth table.

\section{H matrix computed for the complex signal}

We saw how the $\mathbf{W}$ matrix obtained on factorization of the fixed and variable length window STFT compare against each other. Let us see if we have an improvement in the $\mathbf{H}$ matrix as well. Fig. 25 and Fig. 26 plot the gain vectors in the $\mathbf{H}$ matrix obtained on factorization of the fixed and variable length window STFT respectively. 


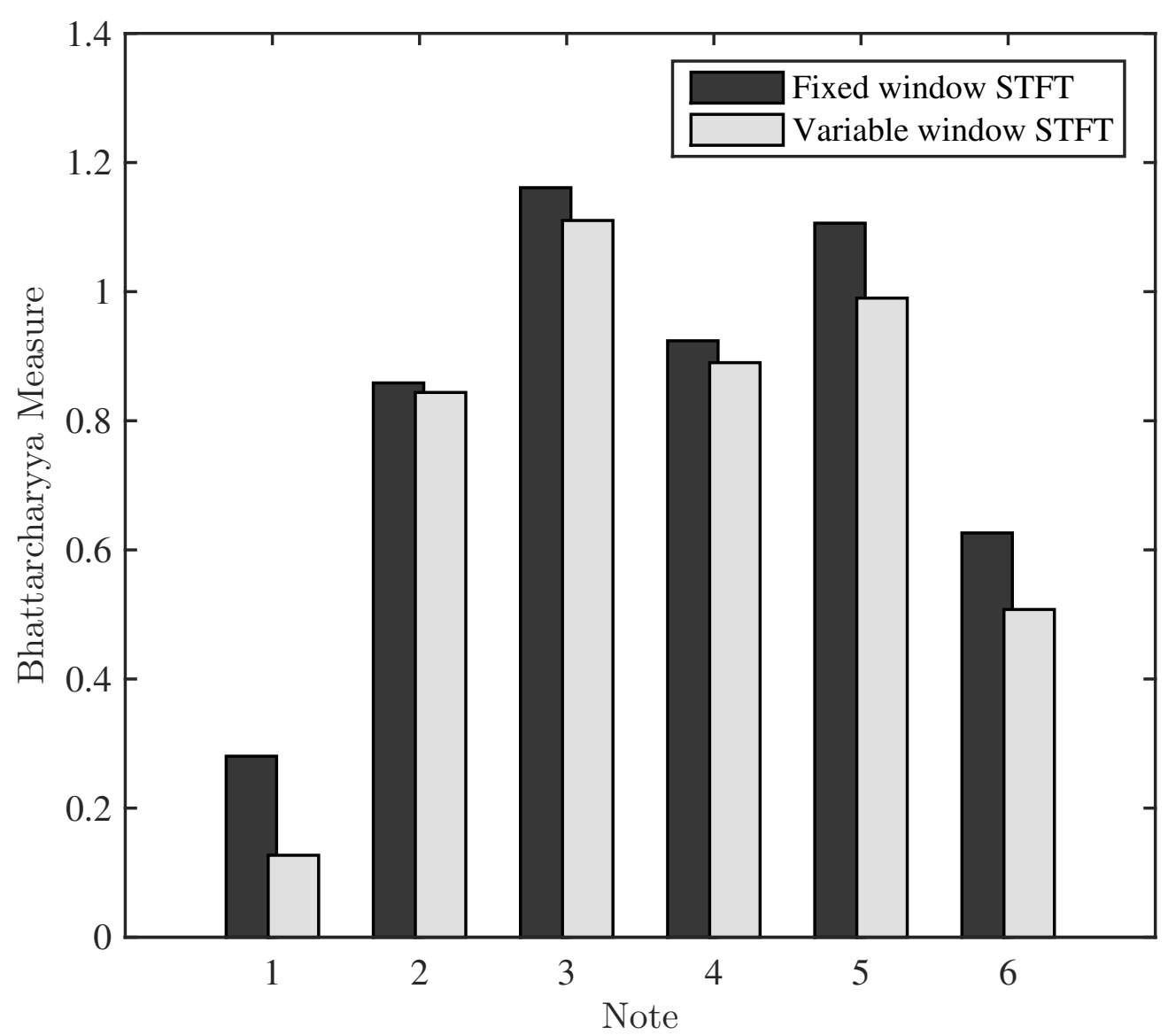

Figure 24: Bar plot of similarity measure of derived notes with ground truth notes using Bhattacharyya distance.

These gain vectors in Fig. 25 and Fig. 26 are ordered in sequence for ease of comparison. From Fig. 25 and Fig. 26 we see that notes 3, 4, 5 and 6 look similar for variable length window STFT and fixed length window STFT. Note 1 looks very similar for both the variable and the fixed length window STFT. We also notice some additional noise that is picked up the gain vector corresponding to the note 2 from the variable length window STFT. The gain of this noise is small and can be easily distinguishable from the true note gain. Now we look at gain vector corresponding to note 1 from Fig. 25 and Fig. 26. The gain vector corresponding to the note 1 of the fixed length window STFT has very spiky structure which is highly uncharacteristic of a note. We can see that the gain vector corresponding to the note 1 of the variable 


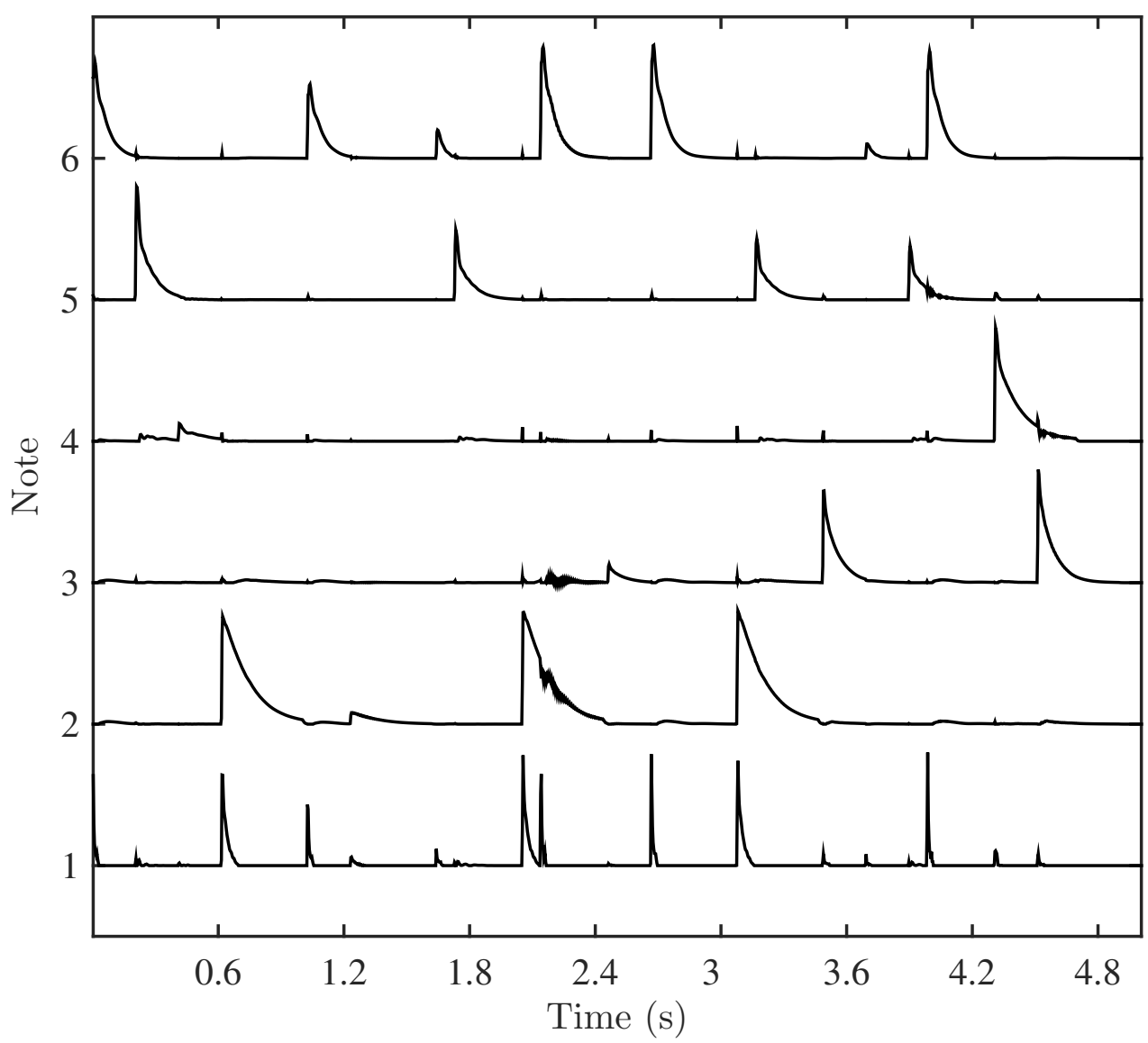

Figure 25: Gain vectors of notes obtained from the factorization of the fixed length window STFT.

length window STFT is a much better approximation of the original note structure.

\subsubsection{Perceptual Evaluation of Derived Notes}

We reconstruct the audio signal using the approximate variable length window based STFT from $\mathbf{W}$ and $\mathbf{H}$ matrices. The phase information from both fixed and variable length window based STFT is used, as the STFT reconstructed from $\mathbf{W}$ and $\mathbf{H}$ matrices only represents the magnitude STFT. Objective scores are calculated using the PEAQb toolbox, an implementation of the ITU-R BS.1387-1 standard to get a perceptual score for the reconstructed audio. The PEAQb toolbox [57] evaluates the perceptual audio quality of the reconstructed signal and maps it to the Objective 


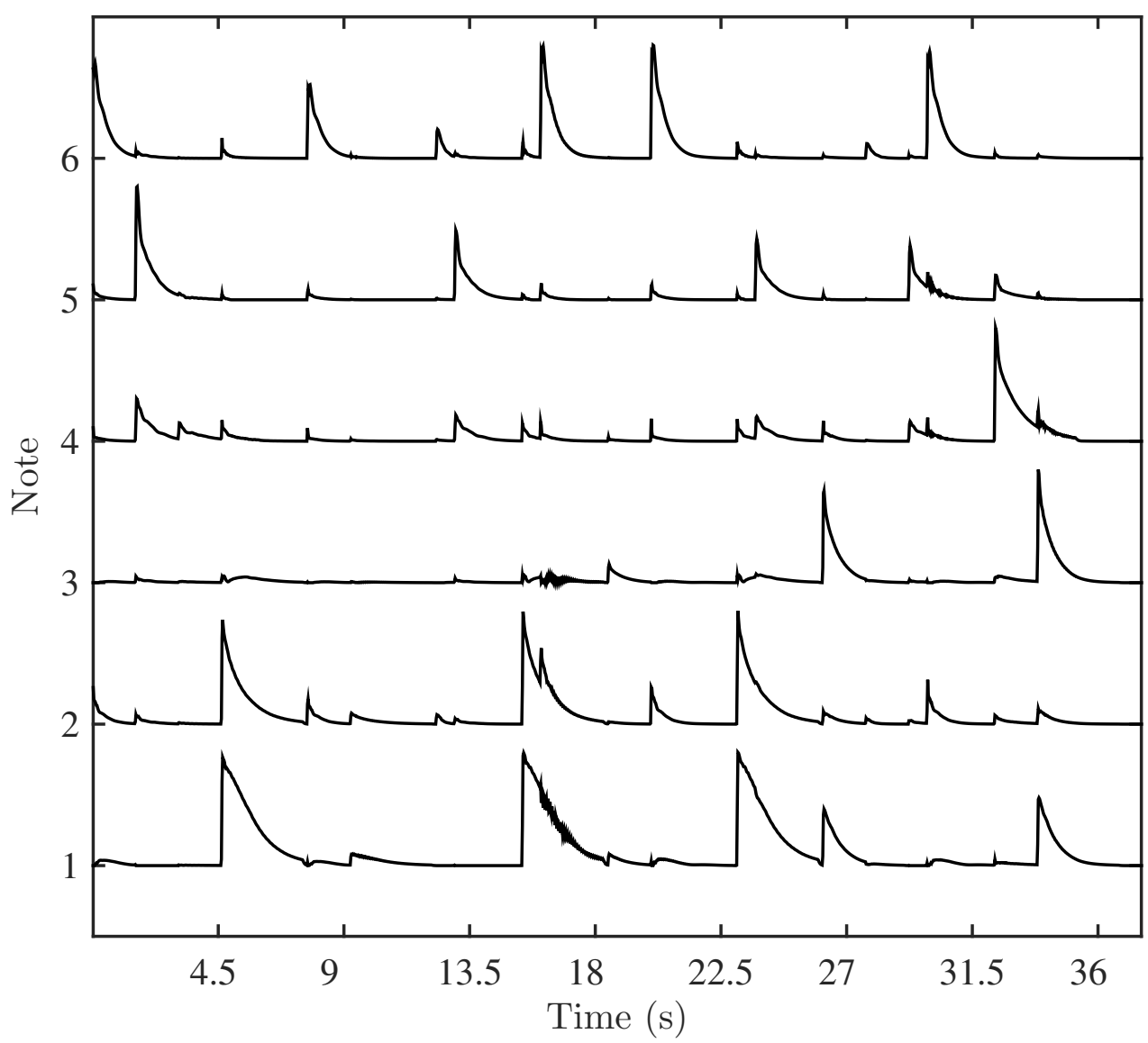

Figure 26: Gain vectors of notes obtained from the factorization of the variable length window STFT.

Difference Grade (ODG). The ODG scale is shown in Table 12.

The reconstructed audio from variable length window based STFT is observed to have clicking sound at frame edges. This is due to discontinuities that are present at frame edges. This clicking noise is also observed with fixed length window based STFT without overlap, and is not observed in fixed length window based STFT, when overlapping windows are used. Overlapped windows help in smoothing the signal at frame edges, thereby reducing discontinuities. Hence, in order to analyze PEAQb results from a neutral perspective, we use overlapping widows in the reconstruction of variable length window based STFT. We first approximate the magnitude variable 
Table 12: ITU-R five-grade impairment scale

\begin{tabular}{|c|c|}
\hline ODG Scale & Perceptual Quality \\
\hline+ ve & Identified as same \\
\hline 0 & imperceptible \\
\hline-1 & Perceptible but not annoying \\
\hline-2 & Slightly annoying \\
\hline-3 & Annoying \\
\hline-4 & Very Annoying \\
\hline
\end{tabular}

Table 13: PEAQb scores for the reconstructed audio signal from $\mathbf{W}$ and $\mathbf{H}$ matrices

\begin{tabular}{|c|c|c|}
\hline File & Fixed STFT & Variable STFT \\
\hline 1 & -3.88206 & -3.46216 \\
\hline 2 & -3.88436 & -3.87115 \\
\hline 3 & -3.72361 & -3.51524 \\
\hline 4 & -3.91030 & -3.90900 \\
\hline
\end{tabular}

length window based STFT from the product of $\mathbf{W}$ and $\mathbf{H}$ matrices. Phase information calculated previously is used to compute the complex STFT. We then apply inverse variable length window STFT to give us frames of audio data. These frames are an approximation of music data and Hamming window. The overlapping frames are segmented such that, they consist of half the samples from windows on either side. These redundant frames are now multiplied with Hamming window. The Hamming windowed segments of data are now added with the overlapping windows to get the reconstructed music signal. This ensures that the overlapping windows contribute most at the frame edges and least at the centre of the frame. It is observed that this arrangement renders the clicking noise imperceptible.

To compare the reconstructed signal with the original signal, we select 4 music files. They are Beethoven's i) Elise and ii) Les Adieux, Clean Bandit's iii) Real love and iv) Bach's Bouree. Beethoven's Elise and Les Adieux are played using piano, Clean Bandit's Real love is pop music with multiple instruments and Bach's Bourree is has both violin and trumpets. The MIDI files for the mentioned music is used to generate the recorded music in GarageBand 10. From Table 13, we note that PEAQb scores are relatively less for the variable length window based STFT. The scores in 
Table 13 are between -3 and -4 , as the signal is reconstructed from an approximation of magnitude STFT.

\subsubsection{Understanding the Results}

In our first experiment we used a simple artificial signal with 6 unique notes, each occurring only once in the test signal and having a significant overlap. In this experiment we see an improvement in the spectra of notes obtained on factorization of the variable length window STFT as compared to the fixed length window STFT. We also see that the gain vectors improve with the proposed method. We can now say that the proposed method works well for this particular signal. In order to generalize the results to represent more like the real world recordings, we run the second the experiment with the complex signal. In this experiment, a complex signal is generated that looks more like the real world recordings. In this signal we have 6 unique notes randomly occurring to give a total of 20 notes. These 20 notes are randomly scaled with varying degrees of overlap. From Fig. 22 and Fig. 23 we can see that the frequency distribution within the notes from the $\mathbf{W}$ matrices obtained on factorized of the fixed and variable length window STFT are very much alike, except for spectral leakage. We see a lot of spectra leakage in the spectra of the notes obtained on factorization of the fixed length window STFT. And this spectral leakage is reduced in the the extracted note spectra obtained on factorization of the variable length window STFT. This can also be seen in the bar chart presented in Fig. 24. We also notice that the results have improved only by a small factor for the complex signal. This could be due to the large number of windows computed for the fixed length window STFT. Due to the large number of overlapping windowing, we can assume that one of the many windows over a note might have captured the pseudo stationary part of the note. As a result the decomposition of fixed length window is similar to the result of decomposing the variable length window STFT. At this point, the variable length window STFT is still doing better than the fixed length window STFT. We perform the third experiment in order to generalize this result. We select 4 pop music files, each file about 3 to 4 minutes to generalize the results of the note extraction. We reconstruct the signal from the factorized parts to get an approximation of the original signal. This approximate signal is then fed to the PEAQb algorithm and the results obtained are tabulated in Table 13. We see that the variable length widow STFT is performing better in general. This leads us to the conclusion that for music 
signals with long duration notes, the performance of the fixed length window STFT will be similar to that of the variable length window STFT. And for music signals with shorter duration notes, the performance would improve with the variable length window STFT, as there will be fewer window that might be able to capture the pseudo-stationary part of the signal in the fixed length window STFT. 


\section{Chapter 5}

\section{Conclusion and Future Work}

\subsection{Itakura-Saito Divergence Based Onset Detec- tion}

The advantage of Itakura-Saito divergence based note onset detection is that it has relatively high true positive onset detection rates when compared with other widely used techniques. Also, since it does not make any assumption about the harmonic nature of underlying music, it can be applied to a wide variety of music signals with different instruments. The disadvantage of Itakura-Saito divergence based onset detection comes from large number of false onset detections, decreasing the F-measure. Overall, the Itakura-Saito divergence based note onset detection works relatively well over a wide range of music irrespective of the instruments in the music signal.

\subsection{Variable Length Window Based STFT}

The goal of this work was to determine whether capturing the stationarity of the signal in the STFT results in improving the quality of derived notes from NMF decomposition. Experiments were performed with MIDI synthesized signals and the frequency spectrum of signal dependent variable length window STFT is compared with the fixed length window based STFT for the NMF approach. It is seen that capturing the stationarity of the signal did indeed improve the quality of notes extracted. Bhattacharyya distance is used to measure the similarity between derived notes (basis vectors in $\mathbf{W}$ ) and the original notes. The Bhattacharyya distance is low for variable length windows, suggesting a nearer approximation of the basis vectors 
in the $\mathbf{W}$ matrix. These results are also verified using perceptual evaluation of audio quality (using the PEAQb toolbox) to compare the reconstructed signal to the input signal. The advantages of using a variable length window based STFT are

1. Reduced spectral leakage in the basis vectors of $\mathbf{W}$ matrix.

2. Reduced number of frames resulting in reduced amount of computational resources and time consumed,

3. Good quality gain vector of corresponding basis vectors. Improved discrimination between the basis vectors is shown to improve the quality of gain vectors in $\mathbf{H}$ matrix, and

4. Improved $\mathbf{W}$ and $\mathbf{H}$ matrices, result in good quality reconstruction of original input signal.

This leads us to the conclusion that signal dependent variable length window based STFT improves the quality of basis vectors in $\mathbf{W}$ matrix, which can then be used to obtain good quality gain vectors in $\mathbf{H}$ matrix from the fixed length window based STFT.

\subsection{Contributions}

Some of the contributions of this thesis are

1. A new onset detection technique in music signals based on Itakura-Saito divergence is explored,

2. A signal dependent variable length window based STFT is explored and has shown to improve the quality of basis vectors in NMF decomposition, and

3. It is shown that these improved basis vectors can be used to extract better quality gain vectors in $\mathbf{H}$ matrix.

\subsection{Future Work}

The current Itakura-Saito divergence suffers from high false positive rates. The Itakura-Saito divergence was primarily used to compare a speech spectra with the 
de-noised spectra. Itakura-Saito divergence less than 0.1 suggests that there is imperceptible difference between the two spectra. Using this to adaptively threshold the STFT might help in reducing the false positive rates.

One of the simplest NMF techniques is used to verify and understand how the signal dependent variable length window based STFT works. Many variants of NMF have been proposed for music signal decomposition, and further explorations should consider what other advantages can be gained if signal dependent variable length windowing is used with those variants on NMF. The next step in this research would be to implement the variable length window based STFT with NMF variant such as Itakura-Saito based NMF or Shifted NMF [5,58,59]. Also, a through investigation of variable length window based NMF on a larger dataset, would be helpful.

Sparse matrix representation of input matrix is shown to speed up the convergence of NMF. Sparse representation of variable length window based STFT could further improve the convergence of NMF.

The gain vectors in $\mathbf{H}$ matrix derived using $\mathbf{W}$ matrix from variable length window based NMF, have some gain from other vectors as well. Improving upon the $\mathbf{W}$ might help in improving the overall quality of $\mathbf{H}$ matrix. Also longer pieces of music recordings with large repetition of notes will help in improving the quality of the learned notes. 


\section{List of References}

[1] P. Smaragdis and J. C. Brown, "Non-negative matrix factorization for polyphonic music transcription," in IEEE Workshop on Applications of Signal Processing to Audio and Acoustics, pp. 177-180, 2003.

[2] B. Widrow and S. D. Stearns, "Adaptive signal processing," Englewood Cliffs, NJ, Prentice-Hall, Inc., 1985, 491 p., vol. 1, 1985.

[3] D. D. Lee and H. S. Seung, "Algorithms for non-negative matrix factorization," in Advances in neural information processing systems, pp. 556-562, 2001.

[4] T. Virtanen, "Monaural sound source separation by nonnegative matrix factorization with temporal continuity and sparseness criteria," IEEE Transactions on Audio, Speech, and Language Processing, vol. 15, no. 3, pp. 1066-1074, 2007.

[5] C. Févotte, N. Bertin, and J.-L. Durrieu, "Non-negative matrix factorization with the Itakura-Saito divergence: With application to music analysis," Neural computation, vol. 21, no. 3, pp. 793-830, 2009.

[6] B. Raj and P. Smaragdis, "Latent variable decomposition of spectrograms for single channel speaker separation," in IEEE Workshop on Applications of Signal Processing to Audio and Acoustics, pp. 17-20, IEEE, 2005.

[7] P. Smaragdis, B. Raj, and M. Shashanka, "A probabilistic latent variable model for acoustic modeling," Advances in models for acoustic processing, NIPS, vol. $148,2006$.

[8] M. Cooper and J. Foote, "Summarizing video using non-negative similarity matrix factorization," in Multimedia Signal Processing, 2002 IEEE Workshop on, pp. 25-28, IEEE, 2002.

[9] H. Kim, H. Park, and B. L. Drake, "Extracting unrecognized gene relationships from the biomedical literature via matrix factorizations," $B M C$ bioinformatics, vol. 8, no. Suppl 9, p. S6, 2007.

[10] S. Ferdowsi, V. Abolghasemi, and S. Sanei, "A constrained nmf algorithm for bold detection in fmri," in Machine Learning for Signal Processing (MLSP), 2010 IEEE International Workshop on, pp. 77-82, IEEE, 2010.

[11] C. H. Ding, X. He, and H. D. Simon, "On the equivalence of nonnegative matrix factorization and spectral clustering.," in SDM, vol. 5, pp. 606-610, SIAM, 2005. 
[12] J. Laroche, "Efficient tempo and beat tracking in audio recordings," Journal of the Audio Engineering Society, vol. 51, no. 4, pp. 226-233, 2003.

[13] P. Masri, Computer modelling of sound for transformation and synthesis of musical signals. PhD thesis, University of Bristol, 1996.

[14] C. Duxbury, J. P. Bello, M. Davies, M. Sandler, et al., "Complex domain onset detection for musical signals," in Proceeding of the Digital Audio Effects Workshop (DAFx), no. 1, pp. 6-9, 2003.

[15] S. Hainsworth and M. Macleod, "Onset detection in musical audio signals," in Proceedings of International Computer Music Conference, pp. 163-6, 2003.

[16] C. Duxbury, M. Sandler, and M. Davies, "A hybrid approach to musical note onset detection," in Proceedings of the Digital Audio Effects Conf.(DAFX, '02), pp. 33-38, 2002.

[17] S. Dixon, "Onset detection revisited," in Proc. of the Int. Conf. on Digital Audio Effects (DAFx-06), pp. 133-137, Citeseer, 2006.

[18] S. Ewert, M. Müller, and P. Grosche, "High resolution audio synchronization using chroma onset features," in Acoustics, Speech and Signal Processing, 2009. ICASSP 2009. IEEE International Conference on, pp. 1869-1872, IEEE, 2009.

[19] S. Böck, F. Krebs, and M. Schedl, "Evaluating the online capabilities of onset detection methods," in ISMIR, pp. 49-54, 2012.

[20] L. Daudet, "Transients modeling by pruned wavelet trees," in Proc. Int. Computer Music Conf.(ICMC'01), pp. 18-21, 2001.

[21] M. Purat and P. Noll, "Audio coding with a dynamic wavelet packet decomposition based on frequency-varying modulated lapped transforms," in Acoustics, Speech, and Signal Processing, 1996. ICASSP-96. Conference Proceedings., 1996 IEEE International Conference on, vol. 2, pp. 1021-1024, IEEE, 1996.

[22] F. Eyben, S. Böck, B. Schuller, and A. Graves, "Universal onset detection with bidirectional long short-term memory neural networks," in ISMIR, pp. 589-594, 2010 .

[23] S. Abdallah and M. D. Plumbley, "Unsupervised onset detection: a probabilistic approach using ica and a hidden markov classifier," in Cambridge Music Processing Colloquium, 2003.

[24] S. A. Abdallah and M. D. Plumbley, "Probability as metadata: event detection in music using ica as a conditional density model," in Proc. 4th Int. Symp. Independent Component Analysis and Signal Separation (ICA2003), pp. 233238, 2003.

[25] W.-C. Lee and C.-C. J. Kuo, "Musical onset detection based on adaptive linear prediction," in Multimedia and Expo, 2006 IEEE International Conference on, pp. 957-960, IEEE, 2006. 
[26] J. W. Cooley and J. W. Tukey, "An algorithm for the machine calculation of complex Fourier series," Mathematics of computation, vol. 19, no. 90, pp. 297$301,1965$.

[27] R. B. R. N. Bracewell, The Fourier transform and its applications, vol. 31999. McGraw-Hill New York, 1986.

[28] J. O. Smith, Spectral Audio Signal Processing. http:http://ccrma.stanford.edu/ jos/sasp///ccrma. stanford.edu/ jos/sasp/, 2011. online book, 2011 edition.

[29] L. R. Rabiner and B. Gold, "Theory and application of digital signal processing," Englewood Cliffs, NJ, Prentice-Hall, Inc., 1975. 777 p., vol. 1, 1975.

[30] F. Itakura and S. Saito, "Analysis synthesis telephony based on the maximum likelihood method," in Proceedings of the 6th International Congress on Acoustics, vol. 17, pp. C17-C20, pp. C17-C20, 1968.

[31] A. Cichocki, S. Cruces, and S.-i. Amari, "Generalized alpha-beta divergences and their application to robust nonnegative matrix factorization," Entropy, vol. 13, no. 1, pp. 134-170, 2011.

[32] M. Goto and Y. Muraoka, "Beat tracking based on multiple-agent architecturea real-time beat tracking system for audio signals," in Proceedings of the Second International Conference on Multiagent Systems, pp. 103-110, 1996.

[33] E. D. Scheirer, "Tempo and beat analysis of acoustic musical signals," The Journal of the Acoustical Society of America, vol. 103, no. 1, pp. 588-601, 1998.

[34] A. Klapuri, "Sound onset detection by applying psychoacoustic knowledge," in IEEE International Conference on Acoustics, Speech, and Signal Processing, vol. 6, pp. 3089-3092, IEEE, 1999.

[35] X. Serra and J. Smith, "Spectral modeling synthesis: A sound analysis/synthesis system based on a deterministic plus stochastic decomposition," Computer Music Journal, pp. 12-24, 1990.

[36] S. N. Levine, Audio representations for data compression and compressed domain processing. PhD thesis, Stanford University, 1998.

[37] E. Marchi, G. Ferroni, F. Eyben, S. Squartini, and B. Schuller, "Audio onset detection: A wavelet packet based approach with recurrent neural networks," in Neural Networks (IJCNN), 2014 International Joint Conference on, pp. 35853591, IEEE, 2014.

[38] A. Holzapfel, Y. Stylianou, A. C. Gedik, and B. Bozkurt, "Three dimensions of pitched instrument onset detection," IEEE Transactions on Audio, Speech, and Language Processing, vol. 18, no. 6, pp. 1517-1527, 2010.

[39] J. P. Bello, L. Daudet, S. Abdallah, C. Duxbury, M. Davies, and M. B. Sandler, "A tutorial on onset detection in music signals," IEEE Transactions on Speech and Audio Processing, vol. 13, no. 5, pp. 1035-1047, 2005. 
[40] D. D. Lee and H. S. Seung, "Learning the parts of objects by non-negative matrix factorization," Nature, vol. 401, no. 6755, pp. 788-791, 1999.

[41] S. S. Chen, D. L. Donoho, and M. A. Saunders, "Atomic decomposition by basis pursuit," SIAM journal on scientific computing, vol. 20, no. 1, pp. 33-61, 1998.

[42] M. Elad, Sparse and redundant representations: From theory to applications in signal and image processing. Springer Science \& Business Media, 2010.

[43] I. Jolliffe, Principal component analysis. Wiley Online Library, 2002.

[44] G. H. Golub and C. Reinsch, "Singular value decomposition and least squares solutions," Numerische Mathematik, vol. 14, no. 5, pp. 403-420, 1970.

[45] A. Hyvärinen and E. Oja, "Independent component analysis: algorithms and applications," Neural networks, vol. 13, no. 4, pp. 411-430, 2000.

[46] M. A. Casey and A. Westner, "Separation of mixed audio sources by independent subspace analysis," in Proceedings of the International Computer Music Conference, pp. 154-161, 2000.

[47] C. Uhle, C. Dittmar, and T. Sporer, "Extraction of drum tracks from polyphonic music using independent subspace analysis," in Proc. ICA, pp. 843-847, 2003.

[48] C.-b. Lin, "Projected gradient methods for nonnegative matrix factorization," Neural computation, vol. 19, no. 10, pp. 2756-2779, 2007.

[49] P. Paatero and U. Tapper, "Positive matrix factorization: A non-negative factor model with optimal utilization of error estimates of data values," Environmetrics, vol. 5, no. 2, pp. 111-126, 1994.

[50] M. D. Juan Pablo Bello, Chris Duxbury and M. Sandler, "On the use of phase and energy for musical onset detection in the complex domain," IEEE Signal Processing Letters, vol. 11, pp. 553-556, June 2006.

[51] K. Schutte, "Midi toolbox." https://github.com/kts/matlab-midi.git, 2012.

[52] S. R. Quackenbush, T. P. Barnwell, and M. A. Clements, Objective measures of speech quality. Prentice Hall, 1988.

[53] G. Chen, S. N. Koh, and Y. Soon, "Enhanced Itakura measure incorporating masking properties of human auditory systemorporating masking properties of human auditory system," Signal Processing, vol. 83, no. 7, pp. 1445-1456, 2003.

[54] A. Bhattachayya, "On a measure of divergence between two statistical population defined by their population distributions," Bulletin Calcutta Mathematical Society, vol. 35, pp. 99-109, 1943.

[55] M. W. Berry, M. Browne, A. N. Langville, V. P. Pauca, and R. J. Plemmons, "Algorithms and applications for approximate nonnegative matrix factorization," Computational statistics \& data analysis, vol. 52, no. 1, pp. 155-173, 2007.

[56] "Matlab statistical and machine learning toolbox version 8.04 .0 (r2014b)," 2014. 
[57] T. Thiede, W. C. Treurniet, R. Bitto, C. Schmidmer, T. Sporer, J. G. Beerends, and C. Colomes, "PEAQ-the ITU standard for objective measurement of perceived audio quality," Journal of the Audio Engineering Society, vol. 48, no. 1/2, pp. 3-29, 2000.

[58] R. Jaiswal, D. FitzGerald, D. Barry, E. Coyle, and S. Rickard, "Clustering nmf basis functions using shifted nmf for monaural sound source separation," in Acoustics, Speech and Signal Processing (ICASSP), 2011 IEEE International Conference on, pp. 245-248, IEEE, 2011.

[59] R. Jaiswal, D. Fitzgerald, E. Coyle, and S. Rickard, "Shifted nmf using an efficient constant-q transform for monaural sound source separation," in 22nd IET Irish Signals and Systems Conference, pp. 23-24, 2011. 